\title{
SigSpec User's Manual
}

\author{
P. Reegen ${ }^{\dagger}$ \\ Institut für Astronomie, Türkenschanzstraße 17, 1180 Vienna, Austria
}

\begin{abstract}
SIGSPEC computes the spectral significance levels for the DFT* amplitude spectrum of a time series at arbitrarily given sampling. It is based on the analytical solution for the Probability Density Function (PDF) of an amplitude level, including dependencies on frequency and phase and referring to white noise. Using a time series dataset as input, an iterative procedure including step-by-step prewhitening of the most significant signal components and MultiSine leastsquares fitting is provided to determine a whole set of signal components, which makes the program a powerful tool for multi-frequency analysis. Instead of the step-by-step prewhitening of the most significant peaks, the program is also able to take into account several steps of the prewhitening sequence simultaneously and check for the combination associated to a minimum residual scatter. This option is designed to overcome the aliasing problem caused by periodic time gaps in the dataset. SIGSPEC can detect non-sinusoidal periodicities in a dataset by simultaneously taking into account a fundamental frequency plus a set of harmonics. Time-resolved spectral significance analysis using a set of intervals of the time series is supported to investigate the development of eigenfrequencies over the observation time. Furthermore, an extension is available to perform the SIGSPEC analysis for multiple time series input files at once. In this MultiFile mode, time series may be tagged as target and comparison data. Based on this selection, SIGSPEC is capable of determining differential
\end{abstract}

\footnotetext{
${ }^{\dagger}$ Note from the Editor: We report with sadness that Peter Reegen, the developer of SigSpec and author of this manual, unexpectedly passed away this year. SigSpec was one of the main achievements of his scientific career. During the previous year he was able to observe the adoption of his program by a large number of astronomers. We sincerely regret the loss of our dear friend and colleague "Piet".

We thank Michael Gruberbauer, who has thoroughly looked through the paper and provided several comments and supplement information. These are represented in uncounted footnotes marked with *

*Note by M. Gruberbauer: Discrete Fourier Transform (DFT)
} 
significance spectra for the target datasets with respect to coincidences in the comparison spectra. A built-in simulator to generate and superpose a variety of sinusoids and trends as well as different types of noise completes the software package at the present stage of development.

\section{What is SigSpeC?}

SigSPEC (abbreviation of 'SIGnificance SPECtrum') is a program that computes a significance spectrum for a time series. It evaluates the Probability Density Function (PDF) of a given DFT amplitude level analytically, making use of the theoretical concept introduced by Reegen $(2005,2007)$. The FalseAlarm Probability, $\Phi_{\mathrm{FA}}(A)$, is the probability that an amplitude in the DFT spectrum exceeds a given limit $A$, and is obtained through integration of the PDF (e. g. Scargle 1982). Instead of this frequently used quantity, SigSPEC calculates the spectral significance (abbreviated by 'sig') of an amplitude $A$ by

$$
\operatorname{sig}(A):=-\log \left[\Phi_{\mathrm{FA}}(A)\right] .
$$

E. g., a sig equal to 5 indicates that the considered amplitude level is due to noise in one out of $10^{5}$ cases. $^{*}$ This value is used as the default threshold for the termination of the prewhitening sequence.

SIGSPEC performs an iterative process consisting of four steps ${ }^{1}$ :

1. computation of the significance spectrum,

2. exact determination of the peak with maximum sig,

3. a MultiSine least-squares fit of the frequencies, amplitudes and phases of all significant signal components detected so far,

4. prewhitening of the sinusoidal components. The residuals are used as input for the next iteration.

If SIGSPEC is called without any special settings, it produces four files:

1. the DFT amplitude spectrum s000000. dat of the original time series, containing also sig and phase,

2. the DFT amplitude spectrum resspec.dat of the residual time series after prewhitening all significant signal components, containing also sig and phase,

* Note by M. Gruberbauer: Note that this quantitiy is defined for a single frequency rather than for the whole spectrum. See Section 15.5. for a discussion.

${ }^{1}$ The AntiAIC computation (p. 47) differs slightly from this procedure. 
3. the residual time series residuals. dat after prewhitening all significant signal components,

4. a result file called result. dat, which contains a list of significant signal components,

5. MultiSine track files, each of which contains a list of the frequencies, amplitudes and phases for a single sinusoidal component through the prewhitening cascade (pp. 36, 87).

Further options may be applied to obtain spectra, residuals, and/or result files (p. 95) in the prewhitening sequence. The MultiSine fits, which are performed after each prewhitening step, modify the frequencies, amplitudes and phases of previous components. If the user examines the resulting signal components and decides not to use all of them, the additional result files help to have accurate frequencies, amplitudes and phases in hands also for a shorter list of significant sinusoids without re-running the program.

SigSPEC can produce additional files containing

1. a spectral window for the given time series (pp. 29, 96),

2. a sampling profile (pp. 30,90) containing the parameters $\alpha_{0}(\omega), \beta_{0}(\omega)$, $\theta_{0}(\omega)$ determining the dependency of the sig on the time-domain sampling, as well as on frequency and phase in Fourier space (see Reegen 2007),

3. a preview of the SIGSPEC analysis (pp.39, 90),

4. a Sock Diagram (pp. 31, 94),

5. a Phase Distribution Diagram (pp. 34, 89) containing probability densities for the Fourier phases,

6. a correlogram for each step of the prewhitening sequence (pp. 41, 86).

These options are deactivated by default.

Given a sequence of prewhitenings yielding $N$ significant components with associated sigs sig $\left(A_{n}\right)$, it is desirable to additionally know the probability of the entire sequence to be valid. This means that not a single erroneous component is allowed. The False-Alarm Probability $\Phi_{\mathrm{FA} n}=10^{-\operatorname{sig}\left(A_{n}\right)}$ of an individual peak is the probability that it is generated by noise. The complementary probability that the considered peak is true is $1-10^{-\operatorname{sig}\left(A_{n}\right)}$. If the individual components are statistically independent, the cumulative probability of all components to be real is the product of the individual probabilities,

$$
1-\Phi_{\mathrm{FA}}=\prod_{n=1}^{N}\left(1-\Phi_{\mathrm{FA} n}\right)
$$


Consistently, the cumulative sig is introduced as the negative logarithm of this total False-Alarm Probability for all identified signal components, $\Phi_{\mathrm{FA}}$, and in terms of individual sigs, one obtains

$$
\operatorname{csig}\left(A_{N}\right):=-\log \left\{1-\prod_{n=1}^{N}\left[1-10^{-\operatorname{sig}\left(A_{n}\right)}\right]\right\} .
$$

In consistency with the definition of the sig associated with an amplitude in the DFT spectrum, a cumulative sig of 3 means that the prewhitening cascade is entirely true in 999 out of 1000 cases. Or - in other words - in one out of 1000 cases, at least one of the identified components is generated by noise.

Whereas the individual sig of a component in the prewhitening sequence may exceed that of the previously identified maximum, the cumulative sig is a monotone sequence uniquely decreasing with each additional signal component.

The prewhitening loop stops, if no sig level above a pre-defined limit is found. As described in "Program termination", p. 23, there are three different criteria that may be applied to determine the conditions for program termination:

1. the number of iterations in the prewhitening sequence,

2. a lower sig limit for the highest peak in the significance spectrum,

3. a threshold for the cumulative sig related to a combined probability for all detected frequency components.

The program also supports the subdivision of a time series into a set of intervals and the separate analysis of all these parts in order to monitor frequency changes of signal components with time. This method will be called timeresolved analysis. In this case, the output is somewhat richer, as described in "Time-resolved Analysis" (p. 42).

An immanent problem in the analysis of non-equidistantly sampled time series is aliasing. Due to periodic gaps in the data set, a peak in the amplitude spectrum is accompanied by side peaks. Especially if more than one sinusoidal component is present in the data, the superposition of side peaks may produce a maximum amplitude in the DFT spectrum at a frequency that has nothing in common with the true signal frequencies. Such a misidentification usually damages the complete prewhitening sequence from this point on. As pointed out by Reegen (2007), SigSpeC appears less prone to aliasing than the previously used methods, since the noise component is employed into the statistical treatment correctly. However, the superposition mentioned above may also lead to erroneous identifications.

In order to overcome this potential weakness, SIGSPEC supports the simultaneous calculation of more than one signal component simultaneously. Instead 
of picking only the peak associated to maximum sig, a whole set of highest peaks is examined, searching all possible combinations for several iterations in order to obtain the solution providing a minimum rms residual. This function is called AntiAlC (ANTI-ALiasing Correction) mode (p. 47).

There is a second option to examine multiple peaks simultaneously: a nonsinusoidal periodicity is represented by multiple peaks in the DFT amplitude spectrum. One finds a fundamental frequency, plus one or more harmonics the frequencies of which are integer multiples of the fundamental. In astronomical applications, this may occur if shock waves are present in the stellar pulsation or if surface variations are examined. In such a case, it is desirable to take into account not only the fundamental frequency, but also all available harmonics at once. This analysis of harmonics is described on p. 51).

SIGSPEC is capable of analysing multiple time series input files simultaneously. This MultiFile mode (p. 56) speeds up the computation considerably for time series with the same sampling.

A further option is the evaluation of differential significance spectra (p. 58). The user may specify target vs. comparison data among the input files. Then SIGSPEC performs a quantitative comparison of the two groups of time series and returns a measure of the probability that a peak in a target dataset is 'true', taking into account amplitudes and phases at the corresponding frequency in the comparison spectra. In this context, the term 'true' is used in the sense of 'not entirely produced by the same variability as present in the comparison data'.

The examples presented here refer to the sample projects available for download at http://www.SigSpec.org.

\section{How to Run SigSpeC}

\subsection{Projects}

SigSPEC is called by the command line

SigSpec <project>

where <project> is the name (or path, if desired) of the SIGSPEC project. Before running the program, the user has to provide

1. a directory <project> used for the output,

2. a time series input file (see "The time series input file", p. 12).

The project directory and the time series input file have to be located in the same folder. The project directory need not be empty. 


\section{Caution: SigSPEC overwrites existing output files!}

There are two conventions for denominating input files.

1. The standard method is to pass only one time series input file to the program. SigSPEC expects the file to be named <project $\rangle$. dat.

2. For an all-in-one analysis of multiple time series input files, i. e., for running SigSpec in MultiFile mode, a leading six-digit index is expected. In this case, the first file shall be named $000000 .<$ project $>$.dat, the next file is 000001 . pproject>.dat, and so on. For more information on the MultiFile mode, please refer to "MultiFile mode", p. 56.

Furthermore, the user may pass a set of specifications to SIGSPEC by means of a file <project>.ini (see "The .ini file", p. 12). This file is expected in the same folder as the time series input file and the project directory. For specifications not given by the user, defaults are used.

Example. The sample project SigSpecNative provides a run without any additional options. The command line is SigSpec SigSpecNative. The sample input file SigSpecNative.dat (381 data points) represents $V$ magnitudes of IC 4996 \# 89 (Zwintz et al. 2004; Zwintz \& Weiss 2006).

The screen output produced by typing SigSpec SigSpecNative at runtime starts with a standard header.

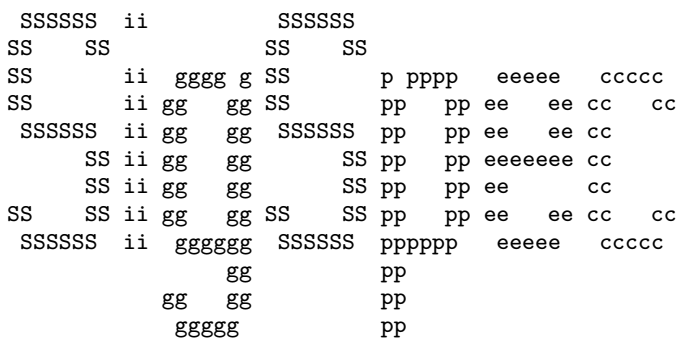

SIGnificance SPECtrum

Version 2.0

****************

Institute of Astronomy

University of Vienna

Tuerkenschanzstrasse 17

1180 Vienna, Austria

Release date: August 18, 2009

SigSPEC processes the command line, checks whether a project directory SigSpecNative is present, and searches for a file SigSpecNative.ini (see 
"The . ini file", p. 12). Since there is no such file present, four warning messages are produced.

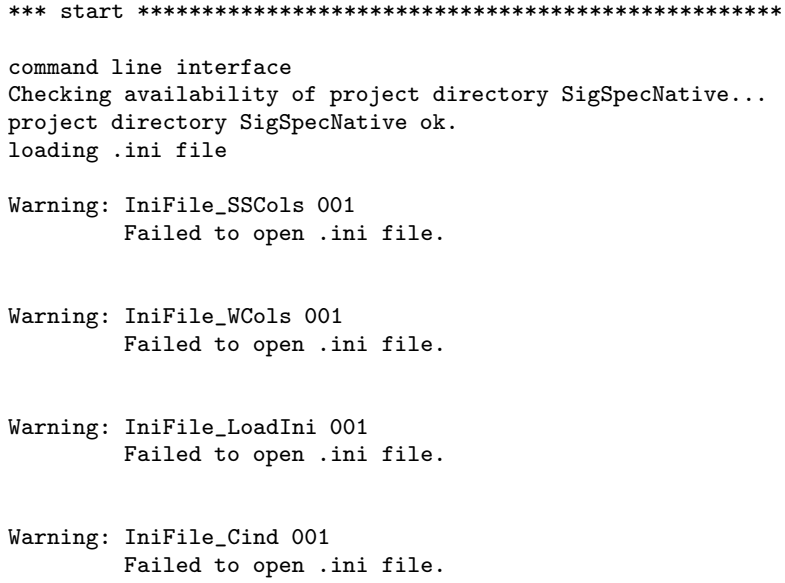

The next task is to load the input file SigSpecNative.dat. SIGSPEC provides the number of rows, the time interval width, and the standard deviation of the observable.

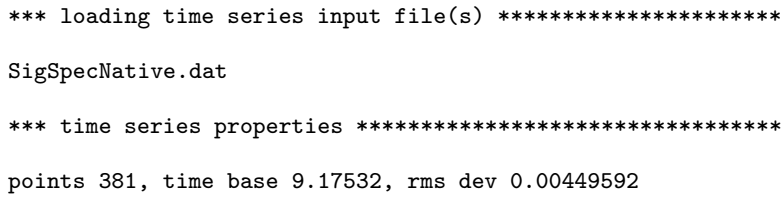

The next section contains the specifications for the DFT and significance spectra to be calculated.

*** preparing to run SigSpec $* *$

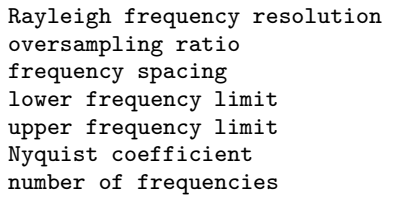

0.1089880382935977

20.0000000000000000

0.0054494019146799

0.0000000000000000

100.4651736990383739

0.5000000000000000 18437

As SigSpeC performs the prewhitening sequence, a list of detected signal components is displayed. The screen output contains the index of the identified component (a line number), the sig, the time-domain rms deviation before prewhitening the corresponding signal, and the csig. The last line contains an insignificant component that meets the breakup condition. In the present example, the default breakup condition (the sig to drop below 5) is applied, which is satisfied in the fourth iteration, where the maximum sig is 4.10802. 
$\begin{array}{llllll}1 \text { freq } 3.13205 & \text { sig } 9.54539 & \text { rms } 0.00449592 & \text { csig } 9.54539 \\ 2 \text { freq } 3.98471 & \text { sig } 7.43085 & \text { rms } 0.00422861 & \text { csig } 7.42753 \\ 3 \text { freq } 5.40684 & \text { sig } 5.30164 & \text { rms } 0.0040257 & \text { csig } 5.2984 \\ 4 \text { freq } 17.3677 & \text { sig } 4.13698 & \text { rms } 0.00388775 & \text { csig } 4.10802\end{array}$

On exit, SigSPEC displays a good-bye message.

Finished.

Thank you for using SigSpec!

Questions or comments?

Please contact Piet Reegen (reegen@astro.univie.ac.at)

Bye!
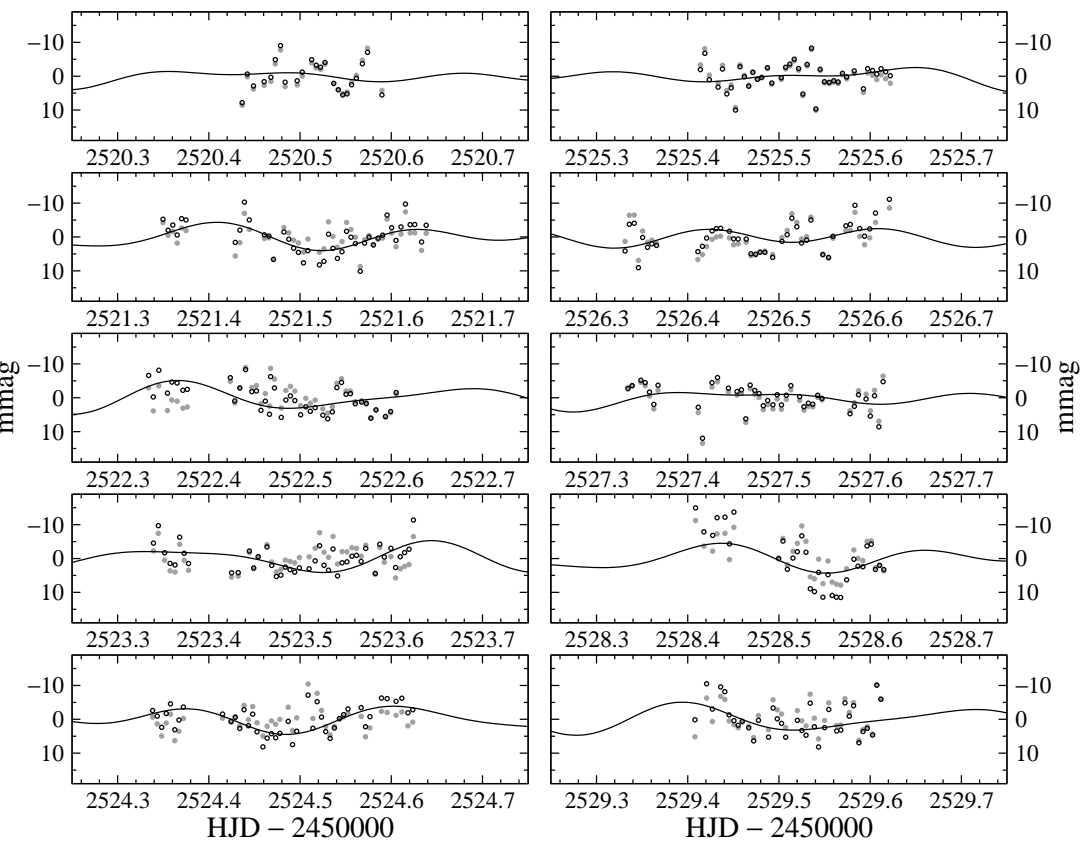

Figure 1: Black circles: light curve for the sample project SigSpecNative. Line: fit formed by three significant signal components (as listed in the file SigSpecNative/result.dat). Grey dots: residuals after prewhitening of three significant signal components (file SigSpecNative/residuals .dat).

If no special output is selected in a file SigSpecNative.ini, SIGSPEC produces the following output files in the project directory SigSpecNative: 


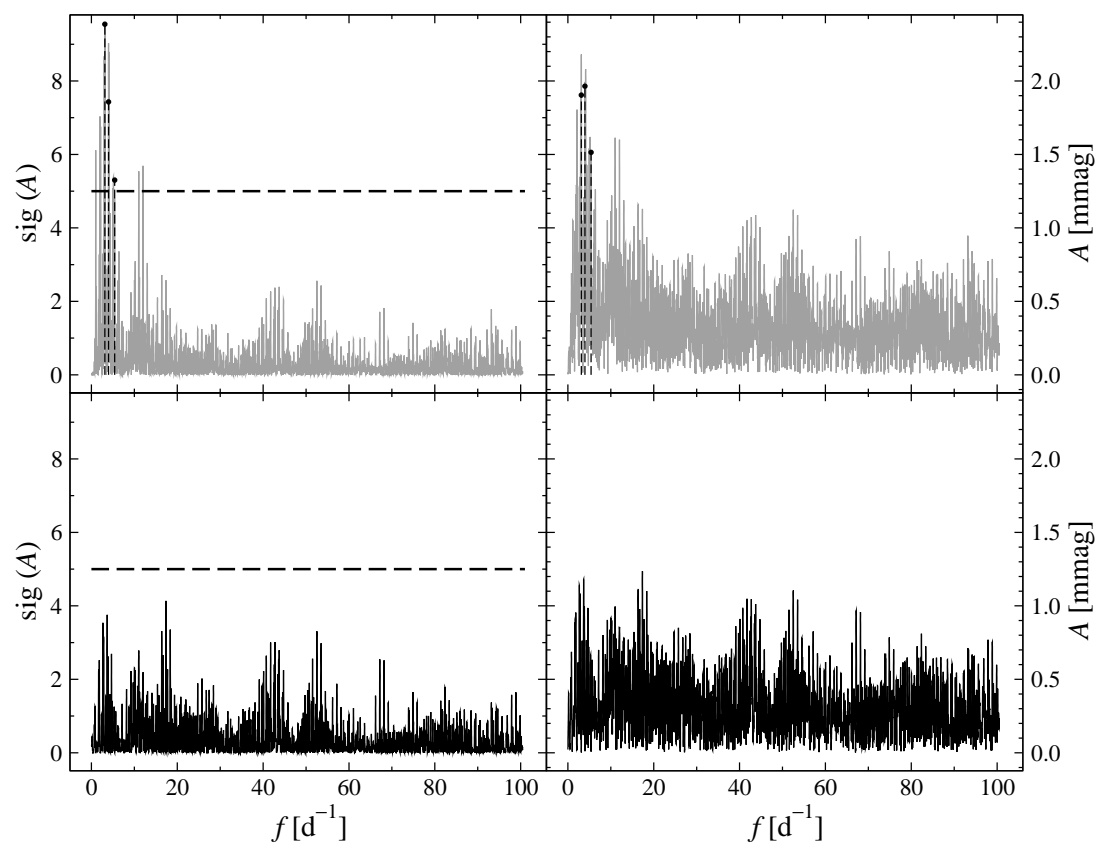

Figure 2: Grey: Fourier spectra for the sample project SigSpecNative. Left: significance spectra. Right: DFT amplitudes. Top: original spectra, without prewhitening (file SigSpecNative/s000000.dat). Bottom: residual spectra, with three significant signal components prewhitened (file SigSpecNative/resspec.dat). In the top panels, the significant components are indicated by dots with dashed drop lines (file SigSpecNative/result.dat). The default sig threshold of 5 is represented by a horizontal dashed line in the left panels.

- s000000. dat: DFT and significance spectrum of the original time series (without any prewhitening),

- result.dat: list of significant signal components detected in the time series,

- residuals.dat: residual time series after prewhitening all significant signal components listed in result. dat,

- resspec.dat: DFT and significance spectrum of the residual time series residuals. dat.

Fig. 1 contains the sample input SigSpecNative.dat, the multisine fit to the time series according to the list of significant signal components in 
SigSpecNative/result.dat, and the residuals after subtracting the fit from the input time series (file SigSpecNative/residuals.dat). Fig. 2 refers to the frequency domain: the DFT spectrum of the initial time series SigSpecNative/s000000.dat, the three significant signal components contained in SigSpecNative/result.dat, and the residual spectrum in the file SigSpecNative/resspec.dat. For detailed information on the contents of the output files, please refer to "Default Output", p. 24.

Furthermore, the user may pass a set of specifications to SigSpEC in a file <project>.ini (see "The .ini file", p. 12). For specifications not given by the user, defaults are used.

\subsection{Quiet mode}

If the command line is followed by the letter ' $q$ ', i. e.

SigSpec <project> q

all screen output is suppressed.

\section{Input}

\subsection{The time series input file}

The input file for SigSpec is a time series. The corresponding file has to be located in the same folder as the project directory. The only restrictions to the format are that the number of items per row has to be constant for all rows in the file and that columns have to be separated by at least one whitespace character or tab.* Dataset entries need not be numeric, except for the columns specified as time, observable, and weights (p. 13).

\subsection{The .ini file}

An optional file <project>.ini consists of a set of keywords and arguments defining project-specific parameters for SigSpeC. If this file is not present in the same folder as the time series input file(s), SigSpEC uses a set of default parameters. A complete list of keywords is given in "Keywords Reference", p. 85 .

Multiple use of the same keyword or the specification of contradictory keywords causes the software to take into account only the last declaration. There are only three exceptions:

\footnotetext{
* Note by M. Gruberbauer: Caution: SigSPEC does not support the exponential annotation (e.g. $4.234 \mathrm{E} 03$ or $1.0385 \mathrm{e}-03)$ !
} 
1. SigSpEC accepts multiple weights columns specified by col:weights (p. 13),

2. multiple subset identifier columns may be specified by col:ssid (p. 15),

3. the simulator may be used to synthesize multiperiodic signal plus various types of noise upon the given sampling, where the keywords sim:signal, sim:poly, sim:exp, sim:zeromean, sim:serial, sim:temporal, and sim:rndsteps may be used multiply (see "The simulator mode", p. 63).

Caution: SIGSPEC expects a carriage-return character at the end of the file $\langle$ project $\rangle$.ini, otherwise the program may hang!

Lines in the .ini file starting with a \# character are ignored by SigSpEC. This provides the possibility to write comments into the file. Furthermore, additional characters beyond what is expected in a line (keyword plus required number of parameters) is ignored. Thus it is allowed to add comments also at the end of the lines containing relevant information for SIGSPEC.

\subsection{Time series columns representing time and observable}

The keywords col:time and col:obs determine those columns in the time series input file which contain time values and the observable monitored over time, respectively. These columns are required and have to be uniquely specified. Column indices start with 1.

If col:time is not specified, the default value 1 is used. If col:obs is not specified, the default value 2 is used.

Example. The sample project coltimecolobs contains a dataset where the time and observable values are found in columns 2 and 3, respectively. The input time series represents the $V$ photometry of IC 4996 \# 89 (see Example SigSpecNative, p. 8). The file coltimecolobs. ini contains the two lines

col:time 2

col:obs 3

\subsection{Time series columns containing statistical weights}

Furthermore, one or more columns with statistical weights may be specified using the keyword col:weights. The keyword accepts two arguments: the first is the column index, the second is a floating-point value, say $p_{n}$ for the $n$th weights column. Given $N$ weights columns indexed according to $n=1, \ldots, N$, 
the total weight for the $m$ th row is evaluated using the weights $w_{n m}$ in the individual columns according to

$$
\Gamma_{m}:=\prod_{n=1}^{N} \gamma_{n m}^{p_{n}} .
$$

Weights need not be normalised, this is performed by SigSPEC.

Time, observable, and weights columns have to consist of floating-point numbers only. SIGSPEC checks these columns before starting the computations. If a non-numeric entry is found in one of the special columns, the program terminates with an error message.

Caution: SigSpeC does not support the exponential annotation (e.g. $4.234 \mathrm{E} 03$ or $1.0385 \mathrm{e}-03)$ !

Example. The sample project weights contains a dataset with statistical weights in column 3 the squares of which are used by SIGSPEC, as specified by the .ini file entry

col:weights 32

The input time series weights. dat represents the sampling of IC 4996 \# 89 $(V)$, and the magnitudes were synthesized by

1. a sinusoid with frequency 4.68573 cycles per day, amplitude $17.27 \mathrm{mmag}$,

2. Gaussian noise with 25 mmag rms deviation only for the measurements between HJD 2452524 and HJD 2452525,

3. Gaussian noise with 2.5 mmag rms deviation for all other nights.

The resulting light curve is displayed in Fig. 3. Fig. 4 compares the frequency domain output (a closeup for frequencies below 10 cycles per day) with and without employing the weights. Without weights, the peak at 4.7 cycles per day visible, but not the most significant one. Moreover, there is no signal that reaches the sig threshold of $5 .^{2}$

1 freq 5.68136 sig 3.75547 rms 0.088716 csig 3.75547

Column 3 in the time series input file weights. dat contains zeroes for the measurements between HJD 2452524 and HJD 2452525 and values of 1 for the rest. Consequently, in this example, the exponent 2 assigned to the keyword $\mathrm{col}$ : weights in the file weights.ini does not affect the weighting: the result would be the same if, e.g.,

\footnotetext{
${ }^{2}$ The result without weights is found in the project directory noweights.
} 

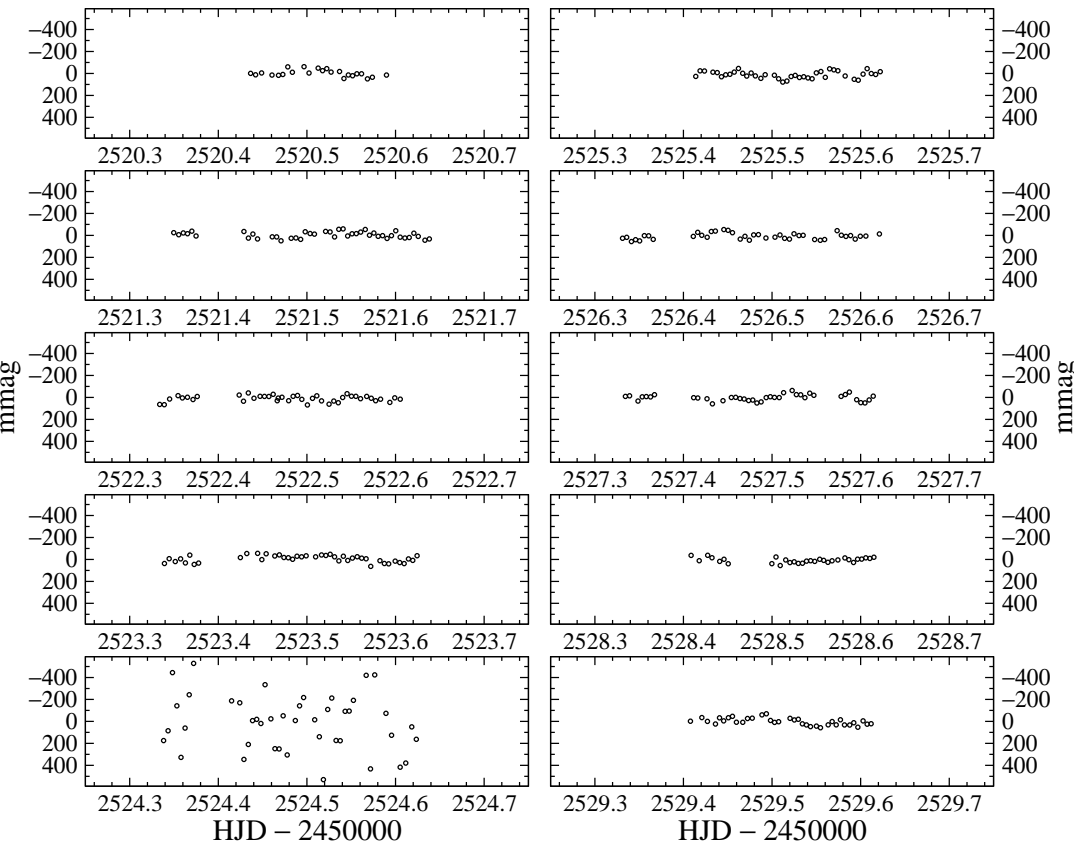

Figure 3: Light curve for the sample project weights.

col:weights 31

were given instead of

col:weights 32

Employing the weights column, SIGSPEC provides the component at 4.7 cycles per day as the only significant signal:

1 freq 4.67968 sig 20.395 rms 0.029129 csig 20.395

2 freq 30.5489 sig 4.47468 rms 0.0252866 csig 4.47468

\subsection{Time series columns containing subset identifiers}

If the mean magnitude of a light curve is desired to be adjusted to zero for each night, or if the data are obtained from more than one site, one may perform an individual zero-mean correction for subsets of the total time series. This is achieved by the keyword col:ssid in the ini file. This keyword is followed by the index of the column which shall be assigned to subset identifiers and may 


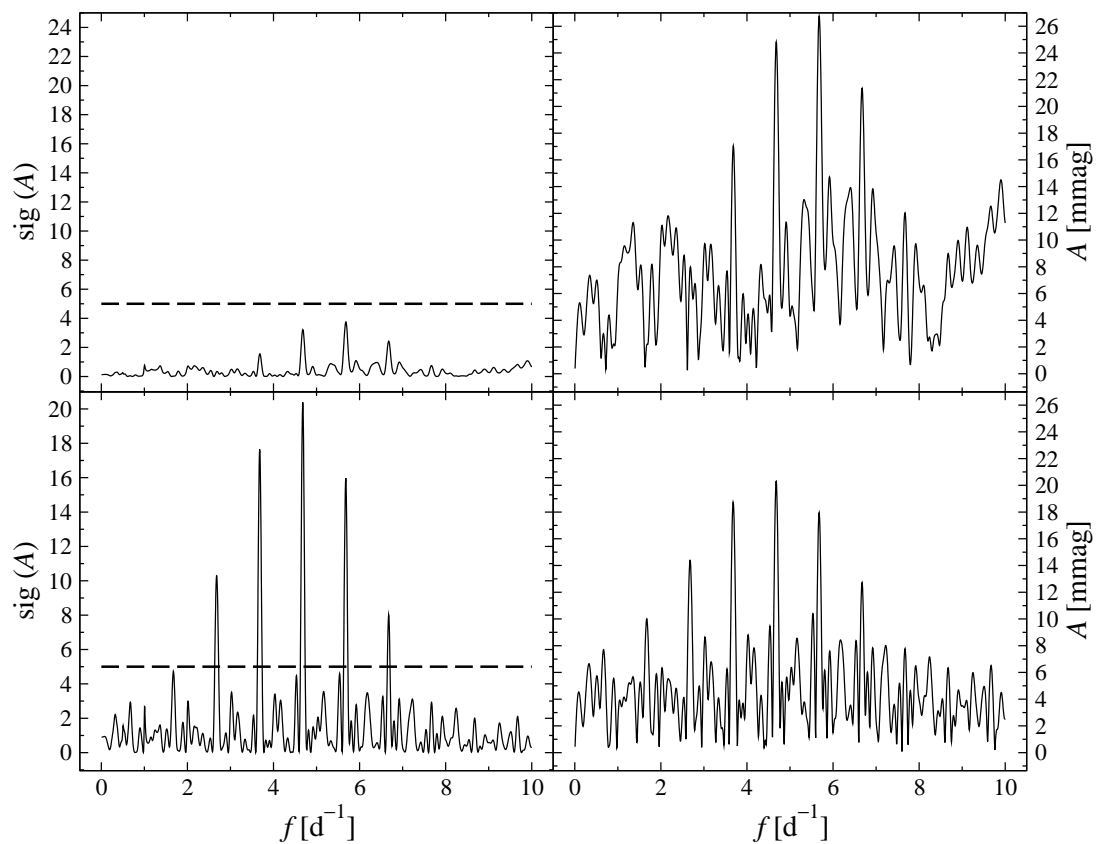

Figure 4: Grey: Fourier spectra for the sample project weights. Left: significance spectra. Right: DFT amplitudes. Top: spectra of the unweighted time series. Bottom: spectra employing statistical weights. The significant components are indicated by dots with dashed drop lines (file weights/result.dat). The default sig threshold of 5 is represented by a horizontal dashed line in the left panels.

be multiply defined, if more than one subset identifier column is given. Subset identifiers may be arbitrary alpha-numeric strings.

If col:ssid is specified, SigSPEC treats all lines in the dataset with equal subset identifiers as individual subsets, for each of which a zero-mean correction is performed. Subsequently, SIGSPEC performs the appropriate statistical calculations, taking into account that the zero-mean correction for subsets diminishes the degrees of freedom for noise.

If more than one subset column is specified, data points are considered to belong to the same subset, if all corresponding subset identifiers are equal.

Example. The sample project subsets contains a dataset with subset identifiers in column 3. The input time series subsets. dat represents the sampling of IC 4996 \# $89(\mathrm{~V})$, and the magnitudes were synthesized by adding

1. Gaussian noise with 5 mmag rms deviation, 

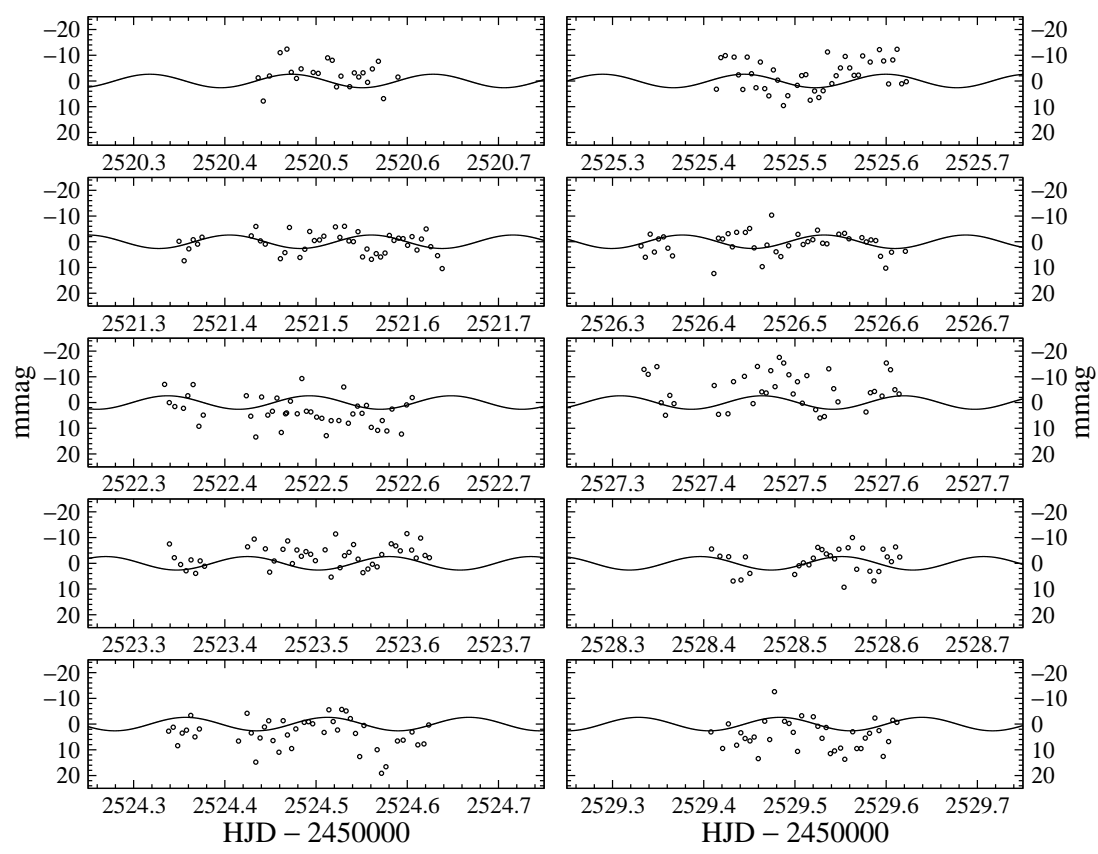

Figure 5: Light curve for the sample project subsets. Solid line: Sinusoidal signal used as input.

2. a sinusoid with frequency 6.43682 cycles per day and amplitude 2.62 mmag,

3. individual constant zeropoint offsets on a millimag range for each night.

The resulting light curve is displayed in Fig. 5, displaying the input signal as a solid line and the data points including the nightly offsets as open dots. Fig. 6 compares the resulting frequency domain output (a closeup for frequencies below 10 cycles per day) with and without employing the weights. If no subdivision according to the subset identifiers is performed, the spectra show a peak at 6.4 cycles per day plus several spurious peaks at frequencies below 2 cycles per day, which are due to the interpretation of the nightly shifts as signal in the 1-cycle-per-day domain and also harmonics. ${ }^{3}$ Consequently, SigSPEC identifies two additional significant signal components at low frequencies:

\footnotetext{
${ }^{3}$ The result without subsets is found in the project directory nosubsets.
} 


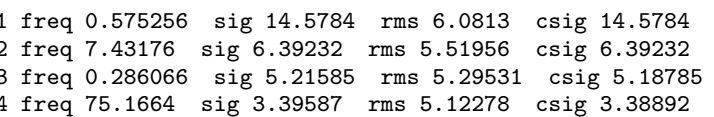

Column 3 in the time series input file weights. dat contains characters $A$ to $J$ uniquely assigned to each night. Employing the subsets column eliminates the low-frequency artefacts, and SIGSPEC provides the component at 6.4 cycles per day as the only significant signal:

1 freq 6.4376 sig 8.20485 rms 5.2253 csig 8.20485

2 freq 75.1661 sig 3.70924 rms 4.95954 csig 3.70922

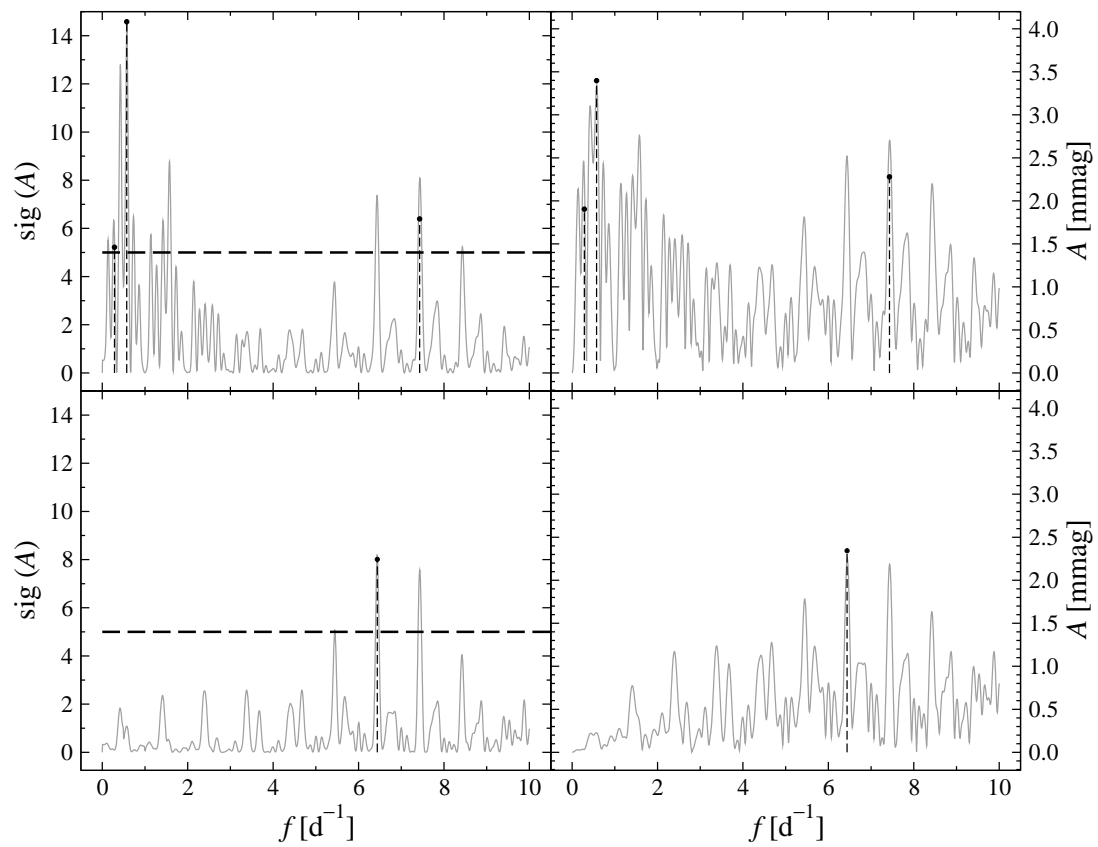

Figure 6: Grey: Fourier spectra for the sample project subsets. Left: significance spectra. Right: DFT amplitudes. Top: spectra of the total time series. Bottom: spectra of the subdivided time series. The significant components are indicated by dots with dashed drop lines (file subsets/result.dat). The default sig threshold of 5 is represented by a horizontal dashed line in the left panels.

\subsection{Lower frequency limit}

The frequency where the computation of spectra starts is specified by the keyword Ifreq. By default, the lower frequency limit is zero. 
Example. The sample project limits illustrates the use of the keyword Ifreq. It uses the $V$ photometry of IC 4996 \# 89 as input file limits. dat, and the file limits.ini contains the line

lfreq 1

which forces SIGSPEC to perform all computations for frequencies $\geq 1$ cycle per day. The spectrum limits/s000000. dat is displayed in Fig. 7.

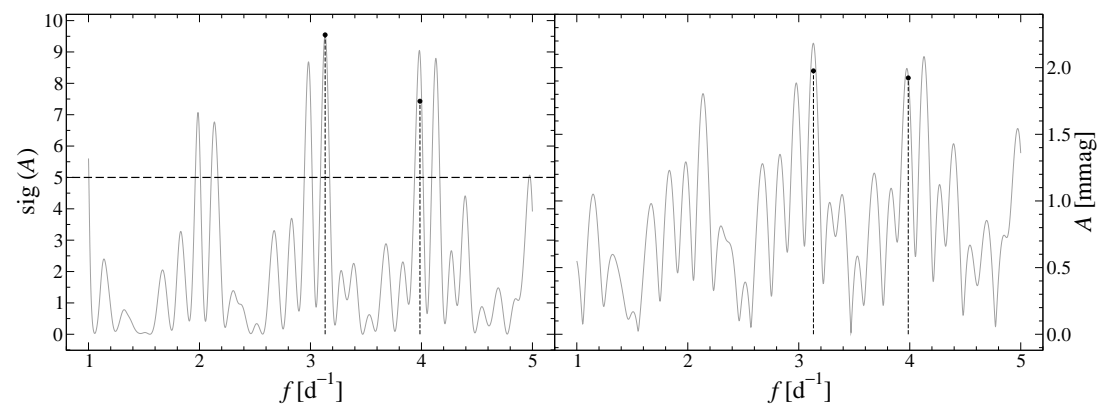

Figure 7: Grey: Fourier spectra for the sample project limits. Left: significance spectrum. Right: DFT amplitudes. The significant components are indicated by dots with dashed drop lines (file limits/result.dat). The default sig threshold of 5 is represented by a horizontal dashed line in the left panel. The frequency range is set from 1 to 5 cycles per day using the keywords lfreq and ufreq.

\subsection{Upper frequency limit and Nyquist Coefficient}

The keyword ufreq allows to determine the upper limit of the frequency interval to be considered.

An alternative method is the automatic determination of this limit by means of the Nyquist Coefficient (keyword nycoef). For equidistantly sampled time series with sampling interval width $\delta t$, there is a uniquely defined Nyquist Frequency

$$
f_{\nu}:=\frac{1}{2 \delta t}
$$

In case of non-equidistant sampling, each sampling interval between two consecutive time values may be considered to produce its individual Nyquist Frequency, whence this limit is ambiguous. In this case, the Nyquist Coefficient for an arbitrarily given frequency is introduced as the fraction of sampling intervals in the time domain the individual Nyquist Frequency of which is higher than the frequency under consideration. This provides to select an upper frequency 
limit by specifying a minimum Nyquist Coefficient. E. g., specifying a Nyquist Coefficient of 0.5 (which is the default value) guarantees that at least half of the information contained by the spectrum in the considered frequency range is below the Nyquist limit.

Additional information is available by setting the keyword nyscan in the .ini file. If this keyword is specified, SigSpec creates a file nyscan.dat in the project directory containing the Nyquist Coefficients over the specified frequency range.

Example. The sample project limits illustrates the use of the keyword ufreq. The line

ufreq 5

in the file limits.ini restricts all computations performed by SiGSPEC to frequencies below 5 cycles per day. The spectrum limits/s000000. dat (sig and amplitude) is displayed in Fig. 7. A comparison with the screen output in Example SigSpecNative, p.9, where no restrictions to the frequency range apply, shows that the screen output in this example contains one line less:

1 freq 3.13205 sig 9.54539 rms 0.00449592 csig 9.54539

2 freq 3.98471 sig 7.43085 rms 0.00422861 csig 7.42753

3 freq 2.664 sig 4.60182 rms 0.0040257 csig 4.60117

The signal component at 5.4 cycles per day is not detected, because it is outside the specified frequency range.

Example. The sample project nyos illustrates the use of the keywords nycoef and nyscan for the $V$ photometry of IC 4996 \# 89 . The line

nycoef 0.99

in the file nyos.ini provides an upper frequency limit of 110.77 cycles per day. The keyword nyscan is given, and the file nyos/nyscan. dat contains the Nyquist Coefficients for frequencies from 0 to 110.77 cycles per day, as displayed in Fig. 8.

\subsection{Frequency spacing and oversampling ratio}

The width of the interval between consecutive frequencies may be specified by the keyword freqspacing.

An alternative method is the automatic determination of the spacing by means of the oversampling ratio. In case of equidistantly sampled time series, the frequency spacing is defined by

$$
\delta f:=\frac{1}{T},
$$




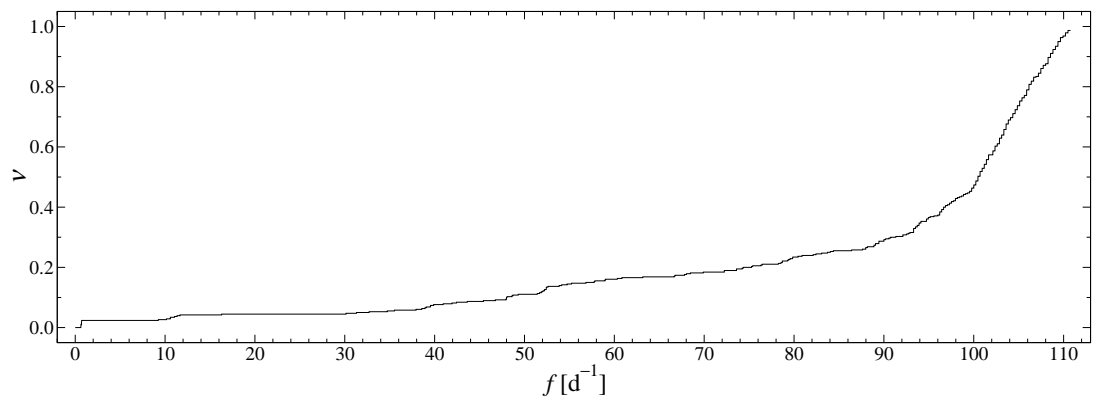

Figure 8: The file nyos/nyscan.dat contains the Nyquist coefficients depending on frequency for the sample project nyos.

where $T$ denotes the width of the total time interval. For non-equidistant time series, it is advisable to use a denser frequency sampling,

$$
\delta f:=\frac{1}{\Omega T},
$$

where $\Omega$ is the oversampling ratio. This quantity may be specified using the keyword osratio. The default value is 20 , which is - in most cases - sufficient for practical use.

Example. The sample project limits illustrates the use of the keyword freqspacing, an example for the keyword osratio is provided in the sample project nyos. Both samples use the $V$ photometry of IC 4996 \# 89 as input time series. The line

freqspacing 0.001

in the file limits. ini forces SIGSPEC to calculate Fourier amplitudes and sigs for every 0.001 cycles per day. In the file nyos. ini, there is a line

osratio 12

which overrides the default oversampling ratio of 20. Fig. 9 compares the standard spacing from Example SigSpecNative, p. 8), with the spacings obtained applying the two above modifications.

\subsection{Accuracy of MultiSine fits}

By default, SIGSPEC performs a MultiSine least-squares fit each time a new significant signal component is detected. The fitting procedure improves the frequencies, amplitudes, and phases of all previously detected signal components. 


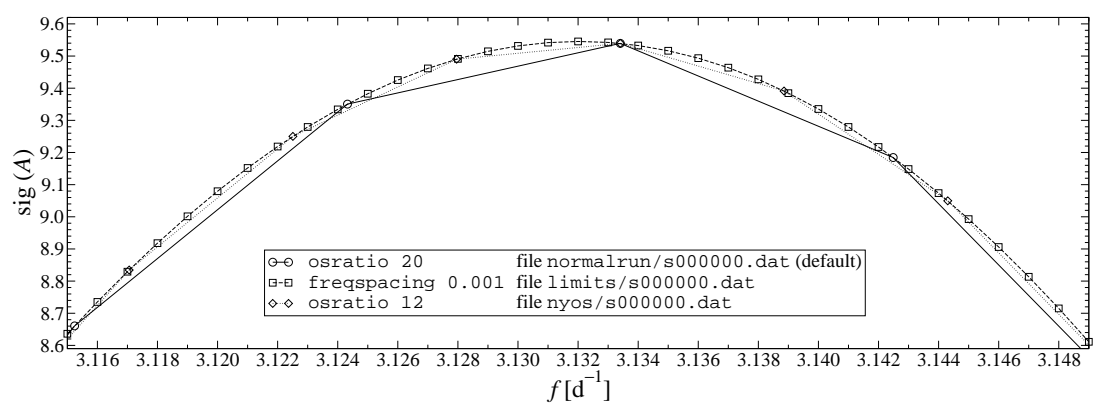

Figure 9: Close-up for the significance spectra generated by the projects SigSpecNative, limits and nyos around the main peak for the $\mathrm{V}$ photometry of IC 4996 \# 89. Different settings for frequency spacing and oversampling ratio are applied.

The algorithm applies Newton's root finding scheme to the first derivatives of the residual variance.

The precision of computed frequencies via MultiSine least-sqares fits is defined according to

$$
\delta f:=\frac{\mu}{T \operatorname{sig}^{\frac{\kappa}{2}}},
$$

where $\mu$ and $\kappa$ are the accuracy parameters for MultiSine fitting. The default value of $\mu$ is $10^{-6}$, that of $\kappa$ is 1 . They may be adjusted by the keyword multisine:newton, followed by $\mu, \kappa$ and a third parameter determining the relative tolerance of the time-domain rms error between consecutive iterations (see next paragraph). To reduce the potential time consumption of the procedure, $\mu$ can be adjusted to achieve an overall scaling of the frequency accuracy. The value of $\kappa$ determines the dependence of the demanded frequency precision on the sig of the peak under consideration. Setting $\kappa=0$ yields the Rayleigh frequency resolution, for $\kappa=1$ one obtains the Kallinger resolution (Kallinger, Reegen \& Weiss 2008).

The criterion on which MultiSine fitting is based is the minimisation of rms residual. Thus the rms residual is demanded to decrease from one iteration to the next. Otherwise the fitting procedure is terminated. To speed up the computation, the MultiSine fit can be terminated, if the relative improvement of rms residual drops below a positive number. The default value $10^{-6}$. This value may be re-adjusted by the third parameter to the keyword multisine:newton.

The two termination conditions are linked by a logical 'and', i. e. the MultiSine fitting procedure stops if both the desired frequency accuracy is reached for all signal components and the improvement of residual rms drops below the specified threshold. 
There is an optional keyword, multisine:lock, that forces the prewhitening cascade to rely on the "raw" frequencies, amplitudes and phases (i.e. those without MultiSine fitting). Resulting signal components are improved to obtain a least-squares fit in each iteration, but this improvement is ignored in the prewhitening sequence. The default setting is that the improved parameters are used for the subsequent analysis (as also obtained by the keyword multisine: unlock.

Example. The sample project multisine illustrates the application of the keyword multisine: newton to the IC 4996 \# 89 photometry (V) as input file multisine.dat. The file multisine. ini contains the line

multisine 0.00100 .01

which reduces the accuracy of the MultiSine fit, compared to the default values $0.000001,1,0.000001$, respectively. The second parameter refers to the Rayleigh frequency resolution rather than the (default) Kallinger frequency resolution. The screen output provides four entries:

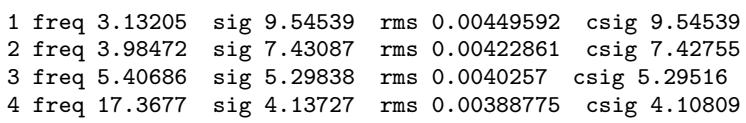

For comparison, the project SigSpecNative, p. 8, employs the default settings.

For the first entry, there is no difference between the two results, but due to propagation of uncertainties, the following entries show slight and increasing deviations. As expected, the rms errors of residuals are higher if the accuracy is reduced.

\subsection{Program termination}

There are three possibilities to specify a criterion for program termination:

1. the number of iterations (keyword iterations),

2. a lower sig limit (keyword siglimit),

3. the reliability of the entire analysis is determined by the cumulative sig. It is based on the probability that at least one of the frequency components detected so far is due to noise. A threshold in terms of cumulative sig may be defined using the keyword csiglimit For an introduction to the cumulative sig, please refer to p. 5. 
Multiple specifications in terms of these keywords cause the prewhitening cascade to terminate if one of the limits is reached.

The default assignment for siglimit is 5 . This pre-definition may be deactivated by defining

siglimit 0

in the .ini file. The limits iterations and csiglimit are switched off by default.

Example. The sample project terminate contains a combination of the keywords siglimit, csiglimit and iterations, applied to the $V$ photometry of IC 4996 \# 89 as input file. For a comparison to the standard output, please refer to Example SigSpecNative, p. 8. The file terminate.ini contains a combination of three keywords:

siglimit 0

csiglimit 3

iterations 10

The first line deactivates the default setting of 5 for the sig limit. The combination of the second and third line forces SIGSPEC to terminate after 10 iterations, or earlier, if the cumulative sig drops below 3. The screen output provides seven lines, corresponding to six significant signal components:

$\begin{array}{llllll}1 \text { freq } 3.13205 & \text { sig } 9.54539 & \text { rms } 0.00449592 & \text { csig } 9.54539 \\ 2 \text { freq } 3.98471 & \text { sig } 7.43085 & \text { rms } 0.00422861 & \text { csig } 7.42753 \\ 3 \text { freq } 5.40684 & \text { sig } 5.30164 & \text { rms } 0.0040257 & \text { csig } 5.2984 \\ 4 \text { freq } 17.3677 & \text { sig } 4.13698 & \text { rms } 0.00388775 & \text { csig } 4.10802 \\ 5 \text { freq } 3.67101 & \text { sig } 3.73187 & \text { rms } 0.00378701 & \text { csig } 3.57943 \\ 6 \text { freq } 52.5182 & \text { sig } 3.41319 & \text { rms } 0.00369756 & \text { csig } 3.18744 \\ 7 \text { freq } 41.7372 & \text { sig } 3.02872 & \text { rms } 0.00361981 & \text { csig } 2.80001\end{array}$

The cumulative sig of 2.8 for component 7 is responsible for program termination before the limit of 10 iterations is reached.

\section{Default Output}

All output files are written into the project directory. A six-digit index denotes the iteration in the prewhitening cascade. E. g., an index 000000 represents a file obtained from the input data without any prewhitening, 000001 denotes a file after prewhitening of the first sinusoidal component. The general annotation \#iteration\# will be used for this six-digit identifier. 
Example. 4 The sample project output illustrates how to adjust the output of SigSPEC. The input file output . dat represents 16 nights (992 data points) of Strømgren y photometry (Vienna University APT, T6; Strassmeier et al. 1997) of the Delta Scuti star EE Cam (Breger, Rucinski \& Reegen 2007). The light curve is displayed in Fig. 10.
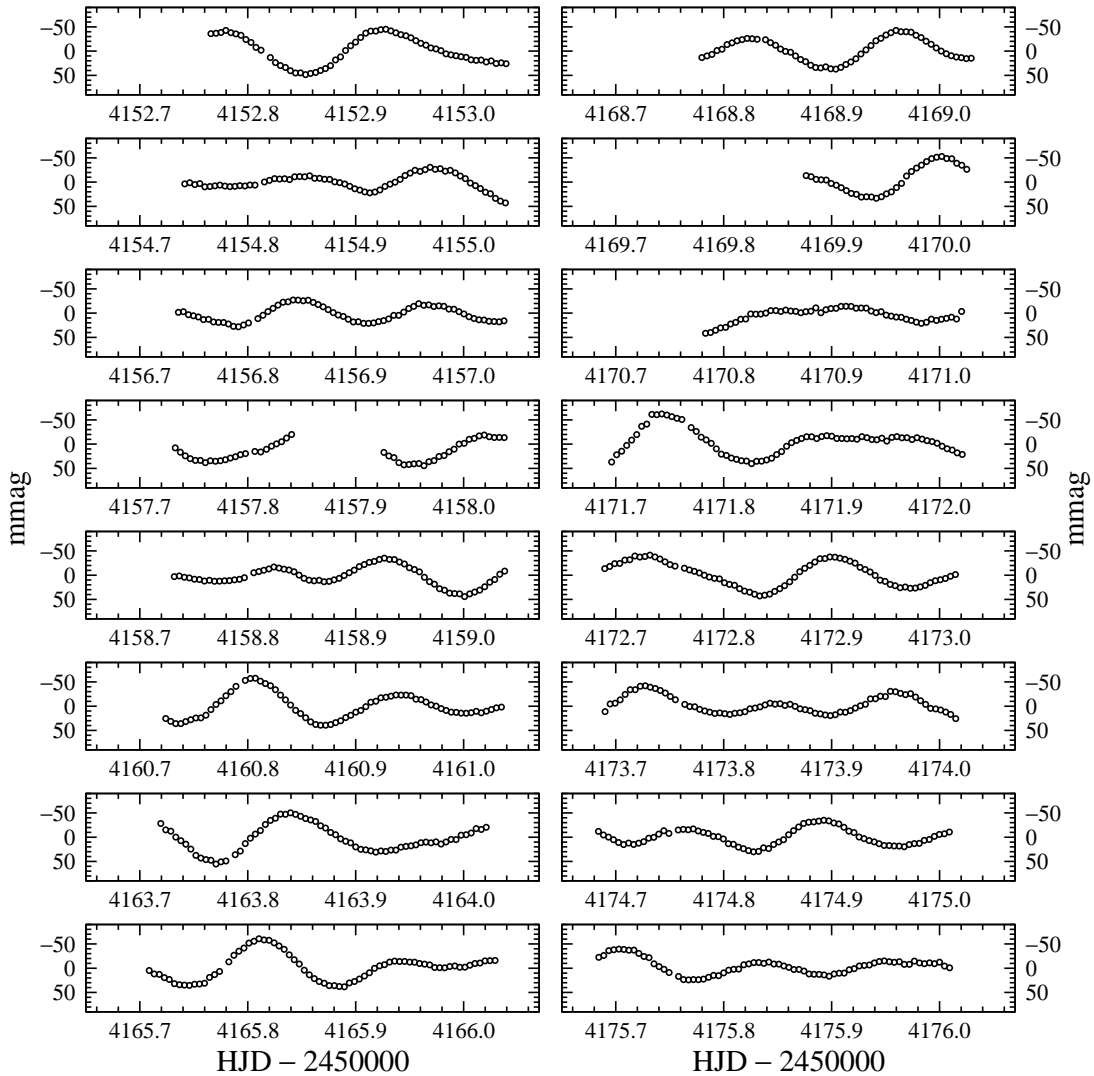

Figure 10: Light curve for the sample project output.

\footnotetext{
${ }^{4}$ The sample project output is the most time consuming sample of all. The computation takes 90 minutes on an Intel Core2 CPU T5500 $(1.66 \mathrm{GHz})$ under Linux 2.6.18.8-0.9-default i686. This is mostly due to the calculations of the Sock and Phase Distribution Diagrams. In order to speed up the program, the user may switch off these operations by placing a \# character at the beginning of all lines containing keywords sock:... and phdist:... in the file output.ini.
} 
The vast amount of output provided by Sock Diagrams and Phase Distribution Diagrams makes it necessary to restrict the frequency interval in the file output.ini. Especially close to zero frequency, the output may be tremendous. Thus the very low frequencies are avoided:

Ifreq 1

ufreq 16

The frequency spacing is adjusted to speed up the computations of Sock and Phase Distribution Diagrams.

freqspacing 0.005

All other entries in the file output.ini apply to output files and are discussed in the subsequent sections.

\subsection{Spectra}

By default, two spectra (files s000000.dat and resspec.dat) are generated. The file s000000. dat contains the spectrum of the original time series, and the file resspec. dat represents the residual spectrum after finishing the prewhitening sequence.

The columns are

1. frequency [inverse time units],

2. sig,

3. DFT amplitude [units of observable],

4. Fourier-space phase angle [rad],

5. Fourier-space phase angle of maximum sig [rad].

To achieve consistency with the output for differential significance spectra (see p. 58), two further columns are found containing values -1 and 0 only.

The phase angles $\theta$ are given according to a trigonometric fit,

$$
F(t):=A \cos (2 \pi f t-\theta),
$$

with amplitude $A$ and frequency $f$ as given in the file. This convention is compatible to the definition of phase in Fourier space. This definition of phase is consistently used for all types of SigSPEC output.

If the keyword spectra is provided in the .ini file, additional output files s\#iteration\#.dat are generated. The index \#iteration\# starts with 000001, denoting the residual spectrum after the first prewhitening step. 
The keyword spectra expects two integer parameters. The first defines the number of iterations for which these files shall be generated. A negative number causes SIGSPEC to generate files for all iterations. The second parameter has to be a positive number and defines a step width. If it is set 1 , a file is generated after each iteration, if it is set 2, after every second iteration (starting with s000002. dat), and so on.

Example. The sample project output uses the keyword spectra in the file output.ini, namely

spectra 102

Spectra are written only during the first 10 iterations of the prewhitening sequence. The second parameter provides only every second file to be generated. In this example, the following files are produced:

output/s000000.dat

output/s000002.dat

output/s000004.dat

output/s000006. dat

output/s000008.dat

output/s000010.dat

In addition, the file resspec. dat contains the residual spectrum after all iterations.

\subsection{Residual time series}

By default, a file residuals. dat is generated. It represents the residuals after prewhitening all signal components found significant. The column format is the same as for the time series input file.

If the keyword residuals is provided in the .ini file, additional files t\#iteration\#. dat are generated, where the index \#iteration\# starts with 000001 , denoting the residuals after the first prewhitening step.

The keyword residuals expects two integer parameters. The first defines the number of iterations for which these files shall be generated. A negative number causes SIGSPEC to generate files for all iterations. The second parameter has to be a positive number and defines a step width. If it is set 1, a file is generated after each iteration, if it is set 2 , after every second iteration (starting with t000002.dat), and so on.

Example. The sample project output uses the keyword residuals in the file output.ini, namely

residuals -15 
Setting the first parameter -1 provides residual time series during the entire prewhitening sequence. The second parameter provides only fifth second file to be generated. Since the number of iterations in this example is 40 , the following files are produced:

output/t000005.dat output/t000010.dat output/t000015.dat output/t000020.dat output/t000025. dat output/t000030.dat

In addition, the file residuals. dat contains the residual time series after all iterations.

\subsection{Result files}

The file result.dat contains a list of all identified sig maxima. This file consists of seven columns providing

1. frequency [inverse time units],

2. sig,

3. amplitude [units of observable],

4. phase [rad],

5. rms scatter of the time series before prewhitening,

6. point-to-point scatter of the time series before prewhitening,

7. the cumulative sig for all frequency components detected so far.

Columns 3 and 4 represent amplitude and phase as the result of a leastsquares fit to the time series at the present prewhitening stage (i. e. after subtraction of all previously identified signal components) for the frequency of maximum significance.

The last line in the file contains zeroes for frequency, amplitude, and phase. The non-zero values refer to the (cumulative) sig of the most significant component below the threshold, and to the rms and point-to-point scatter after the last prewhitening step, respectively. This final line is suppressed if the criterion iterations is responsible for program termination.

If the keyword results is provided in the .ini file, additional result files r\#iteration\#.dat are generated, where the index \#iteration\# starts with 000001 , denoting the result of the first iteration. The files contain the significant components within the prewhitening cascade as preliminary results. The 
MultiSine least-squares fits which are performed at each step of the prewhitening sequence modify frequencies, amplitudes and phases. Therefore it may be useful to have additional results from earlier iterations in hands, if the user decides not to use all components found by SIGSPEC without re-running the program.

The keyword results expects two integer parameters. The first defines the number of iterations for which these files shall be generated. A negative number causes SigSpec to generate files for all iterations. The second parameter has to be a positive number and defines a step width. If it is set 1 , a result file is generated after each iteration, if it is set 2 , after every second iteration (starting with r000002.dat), and so on.

Example. The sample project output uses the keyword results in the file output.ini, namely

results -11

providing result files r000001.dat, r000002. dat,..., for all iterations of the entire prewhitening sequence. In addition, the final result after all prewhitening iterations is contained in the file results. dat.

\section{Analysis of the Time-domain Sampling}

Example. The sample project output contains the output of a spectral window, a sampling profile, a sock diagram, a phase distribution diagram, a preview, and correlograms.

\subsection{Spectral window}

The spectral window is computed, if the keyword win is given in the ini file. This keyword does not require any parameters. The output is provided in the file win. dat. It consists of three columns referring to

1. frequency [inverse time units],

2. amplitude [units of observable],

3. Fourier-space phase angle [rad].

Example. The sample project output contains the output of a spectral window. The file output. ini contains the keyword win, and the corresponding output is found in the file output/win. dat and displayed in Fig.11. The frequency limits determined by the lines 
lfreq 1

ufreq 16

also apply to the spectral window.

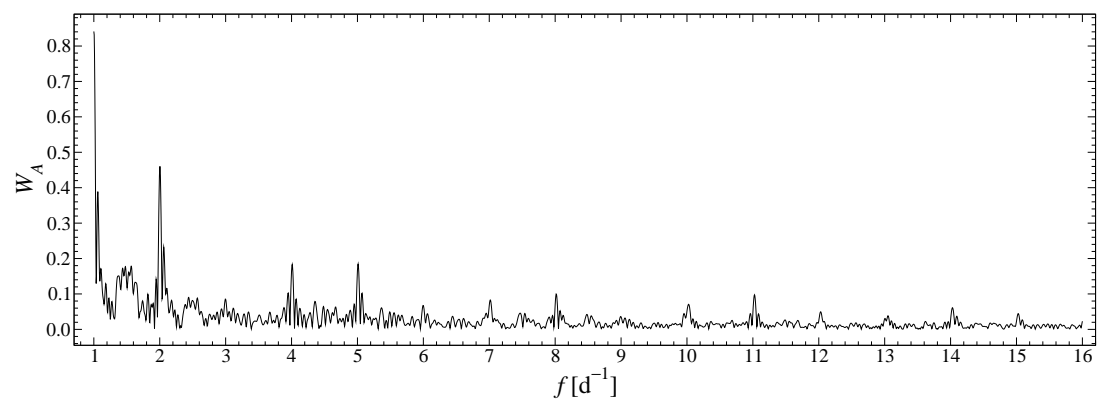

Figure 11: Spectral window for the sample project output.

\subsection{Sampling profile}

The sampling profile is an essential part of the sig computation. All parameters to describe the influence of the time series sampling in Fourier space is entirely contained in the three parameters $\alpha_{0}, \beta_{0}$, and $\theta_{0}$. The values of $\alpha_{0}$ and $\beta_{0}$ are measures for the maximum and minimum sig for all phase angles at a given frequency, and the angle $\theta_{0}$ determines the phase angle where maximum sig is obtained at the frequency under consideration. A detailed description is given by Reegen (2007). If the keyword profile is provided in the .ini file, the sampling profile for the given time series is written to the file profile.dat. The four columns refer to

1. frequency [inverse time units],

2. $\alpha_{0}$,

3. $\beta_{0}$,

4. $\theta_{0}[\mathrm{rad}]$.

Example. In the file output.ini, the keyword profile is given and forces SigSPEC to generate an output file output/profile.dat representing the sampling profile displayed in Fig. 12. 


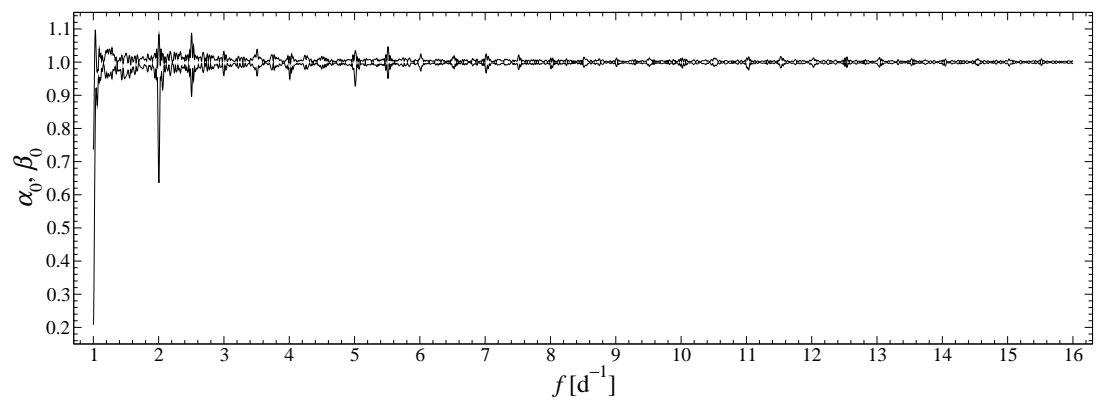

Figure 12: Sampling profile for the sample project output. The lower curve refers to $\alpha_{0}$, the upper curve to $\beta_{0}$. The orientation angle of the rms error ellipse, $\theta_{0}$ is not plotted.

\subsection{Sock Diagram}

The computation of a Sock Diagram is an optional add-on of SigSPEC. If the keyword sock:phases is given in the .ini file, SigSPEC computes sock significances, i. e. sig levels for a constant signal-to-noise ratio at a set of different phase angles, and for all frequencies for which spectra are calculated. As described by Reegen (2007), the expected sig level for a given amplitude signal-to-noise ratio at constant frequency and phase angle is proportional to the squared amplitude signal-to-noise ratio. Sig levels in the Sock Diagram are normalised to an expected value of 1 , corresponding to an amplitude signal-tonoise ratio

$$
\frac{A}{\langle A\rangle}=\frac{2}{\sqrt{\pi \log \mathrm{e}}} \approx 1.712 \text {. }
$$

The sig level for an arbitrary signal-to-noise ratio may be deduced by multiplying the sig displayed in the Sock Diagram by $\frac{\pi \log e}{4}\left(\frac{A}{\langle A\rangle}\right)^{2} \approx 0.341\left(\frac{A}{\langle A\rangle}\right)^{2}$.

Furthermore, the phase angle in the Sock Diagram is given with respect to $\theta_{0}$, i. e. the phases with maximum sock significance are consistently aligned to zero phase for all frequencies.

The number of phase angles in the interval $[0, \pi[$ to be taken into account for each frequency of the spectrum has to be given as an argument to the keyword sock:phases in the .ini file. The sig levels in the phase intervals $[0, \pi[$ and $[\pi, 2 \pi[$ are symmetric according to

$$
\operatorname{sig}(A, \omega, \phi)=\operatorname{sig}(A, \omega, \phi+\pi) \forall \phi,
$$

but both given in the output file sock. dat for completeness. The result represents a three-dimensional polar diagram of the sampling properties of the time 
series input file. To enhance the corresponding plot resolution, the number of phases specified with the keyword sock:phases in the .ini file is scaled by the maximum sig for each frequency. For sig maxima $\leq 1$, the specified number is used, for sig maxima between 1 and 2, the number is doubled, and so on.

To enhance the quality of Sock Diagrams produced by SigSPEC, the keyword sock:fill can be provided to specify a fill factor (as a floating-point number following the keyword). It is used for adaptive oversampling of frequencies according to the differences of maximum sigs for consecutive frequencies. The fill factor is the (rounded) number of additional frequencies per unit of sig difference. In other words, providing sock:fill 10 guarantees that the resolution of the resulting Sock Diagram along the sig axis does not exceed 0.1, and an appropriate combination of the keywords sock: phases and sock:fill produces a Sock Diagram that mimics a continuous surface when plotted in 3D style. The default argument of sock: fill is 0 , which means that adaptive oversampling is switched off.

Caution: the Sock Diagram may become a huge file! Especially for very low frequencies, a tremendous amount of data may be expected. Thus it is advisable either to exclude this frequency region (keyword lfreq) or to assign very low values to sock:phases and sock:fill.

The user may choose to obtain the Sock Diagram in three-dimensional cylindrical (default, or keyword sock:cyl) or cartesian coordinates (keyword sock: cart).

In any case, the output file sock. dat consists of three columns. In cylindrical coordinates, the columns refer to

1. height coordinate: frequency [inverse time units],

2. azimuthal coordinate: phase with respect to the sig maximum [rad],

3. radial coordinate: sock significance.

In cartesian coordinates, the columns refer to

1. frequency [inverse time units],

2. sock significance component in the direction of the sig maximum,

3. sock significance component in the direction of the sig minimum.

The keywords sock:colmodel:lin and sock:colmodel:rank permit to choose between two different colour models assigning RGB colours to the data points of the Sock Diagram. The linear model (sock:colmodel:lin) 
uses the sock significance as it is for colour scaling, whereas the rank model (sock: colmodel:rank) relies on a rank statistics of sock significances.

Caution: the computation of ranks may be very time-consuming! The progress control displayed during the calcucation of the rank statistics does not provide linear percentages in time. The percentage values refer to the portion of ranks among the number of data points that are finished.

A sequence of keywords sock: colour determines a colour path that is assigned to the data points in the Sock Diagram. The keyword is followed by four floating-point arguments. The first three arguments refer to the three RGB channels. Using the linear model (sock: colmodel:lin), the fourth argument is the sock significance to which the given colour has to be assigned. For the rank model (sock:colmodel:rank), the fourth argument is a floating-point value in the interval $[0,1]$ and determines the fractile of data points to which the given colour has to be assigned. A value of, e. g., 0.5 assigns the specified colour to the median of sock significances. SIGSPEC performs a linear interpolation along this colour path and assigns a fourth column to the output file sock. dat containing RGB values. For entries beyond the start or end of the colour path, the start or end colour is used, correspondingly.

Example. A linear colour model that produces colours from white via red, yellow, green, cyan, blue, and magenta to black is produced by the following specifications:

$\begin{array}{lrrrl}\text { sock:colmodel:lin } & & \\ \text { sock:colour } & 255 & 255 & 255 & .5 \\ \text { sock:colour } & 255 & 0 & 0 & .9 \\ \text { sock:colour } & 255 & 255 & 0 & .95 \\ \text { sock:colour } & 0 & 255 & 0 & 1 \\ \text { sock:colour } & 0 & 255 & 255 & 1.05 \\ \text { sock:colour } & 0 & 0 & 255 & 1.1 \\ \text { sock:colour } & 255 & 0 & 255 & 1.2 \\ \text { sock:colour } & 0 & 0 & 0 & 2\end{array}$

Example. A rank colour model producing greyscale coding is obtained by:

sock: colmodel : rank

sock:colour $0 \quad 0 \quad 00$

sock:colour 2552552551

Example. The Sock Diagram in the sample project output is generated according to the following entries in the file output.ini: 

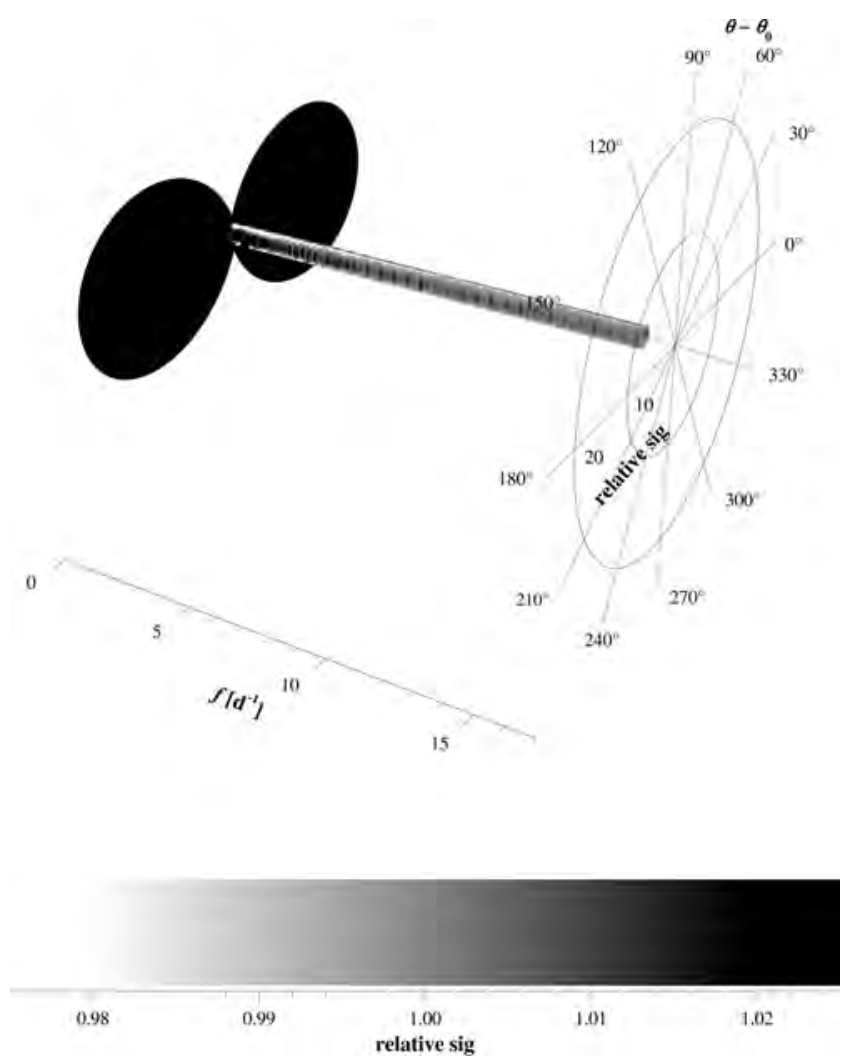

Figure 13: Sock Diagram for the sample project output.

sock : cyl

sock: phases 45

sock:fill 10

sock: colmodel:lin

sock:colour 2552552550.98

sock:colour $0 \quad 0 \quad 0 \quad 1.02$

The resulting file output/sock. dat is displayed in Fig. 13.

\subsection{Phase Distribution Diagram}

In addition to the spectral window and Sock Diagram, SigSpec can compute the probability density of phase angles at given frequency as a function of frequency. This is an alternative way to examine the properties of the sampling in the time domain and activated by the keyword phdist:phases in the .ini 
file. The resulting probability densities are normalised in a way that their mean over all phase angles is $\frac{1}{2 \pi}$.

The number of phases to be computed is increased according to the eccentricity of the phase distribution at a given frequency.

In perfect analogy to the Sock Diagram (p.31), there are further keywords available to adjust the contents of the output file phdist.dat.

- phdist:fill determines a filling factor for additional frequencies if the changes between the phase distributions for two adjacent frequencies are too high.

- phdist : cyl specifies cylindrical coordinates (height: frequency, azimuth: phase, radial: probability density of phase)

- phdist: cart specifies cartesian coordinates

- phdist:colmodel:lin

- phdist:colmodel:rank

- phdist:colour

Please refer to "Sock Diagram" (p. 31) for a detailed description.

Caution: For frequencies close to zero, tremendous output may be expected! Try to avoid the very low frequency region, if possible.

Example. The Phase Distribution Diagram in the sample project output is generated according to the following entries in the file output.ini:

phdist: cart

phdist: phases 30

phdist:fill 50

phdist: colmodel:rank

phdist:colour 2232232230

phdist:colour $31 \quad 31 \quad 311$

The resulting file output/phdist. dat is displayed in Fig. 14.

\section{MultiSine Output}

After each step of prewhitening, SIGSPEC performs a MultiSine least-squares fit over all significant signal components detected so far. Two optional types of output may help the user comprehend how this procedure performs at runtime. 


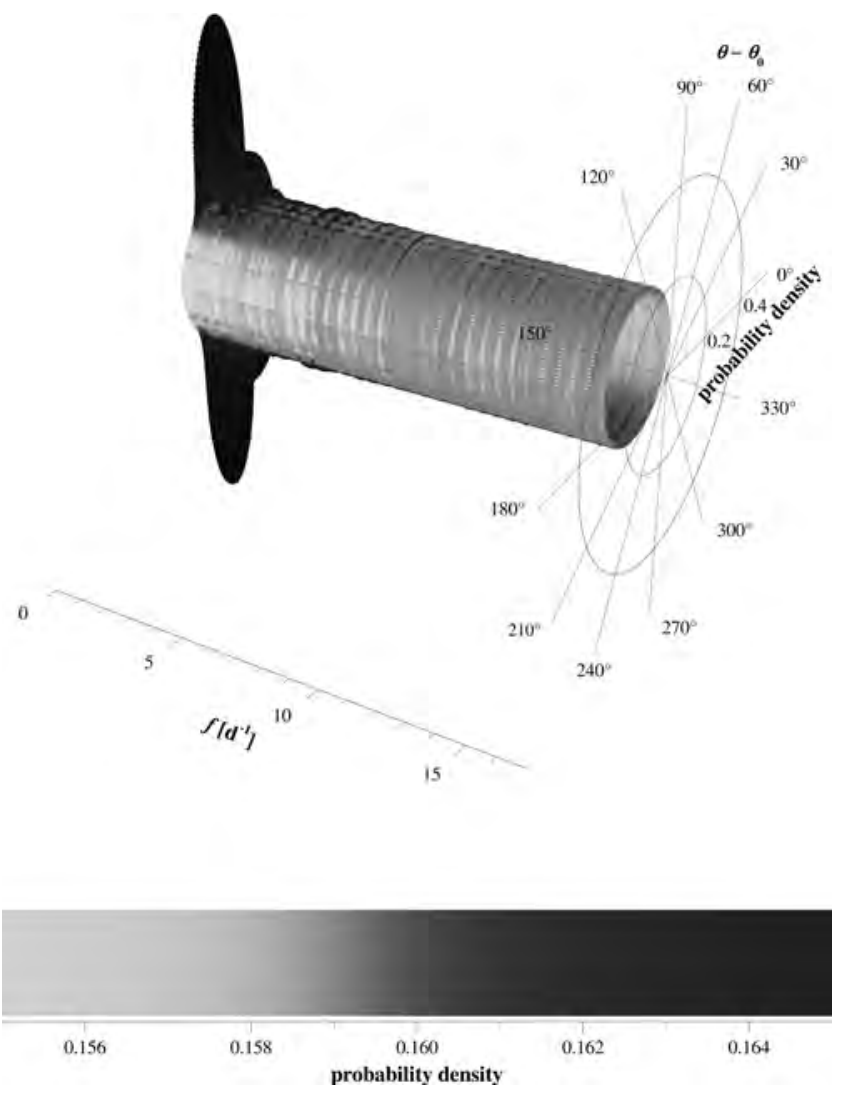

Figure 14: Phase Distribution Diagram for the sample project output.

\subsection{MultiSine tracks}

The MultiSine tracks allow to examine the changes in frequency, amplitude and phase of each signal component in the prewhitening cascade and are an alternative representation of the result files. Instead of a file index that refers to the iteration, the file index of the MultiSine track files m\#index\#. dat refers to the index of the component in the result files and lists its

1. frequency [inverse time units],

2. amplitude [units of observable],

3. phase $[\mathrm{rad}]$ 
for each prewhitening step. In other words, a result file displays all the components for an iteration, whereas the MultiSine track file displays all the iterations for a component. Thus the MultiSine track provides a good estimate for the reliability and accuracy of the components found significant.

If the keyword mstracks is provided in the .ini file, MultiSine track files m\#index\#. dat are generated, where the index \#index\# starts with 000001 , denoting the first significant signal component.

The keyword mstracks expects two integer parameters. The first defines the number of iterations for which entries in the MultiSine track files shall be generated. A negative number causes SIGSPEC to generate entries for all iterations. The second parameter has to be a positive number and defines a step width. If it is set 1 , a line in the MultiSine track files is generated for each iteration, if it is set 2 , for every second iteration (starting with r000002. dat), and so on.

Example. The sample project output uses the keyword mstracks in the file output.ini, namely

mstracks -11

providing MultiSine tracks m000001. dat, m000002. dat,..., for all iterations of the entire prewhitening sequence. The MultiSine track for the primary signal component (file m000001. dat) is displayed in Fig. 15.
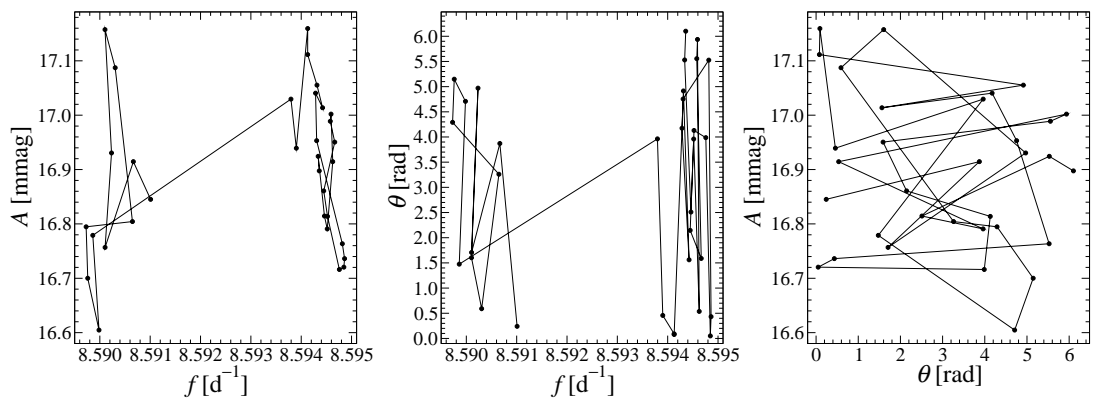

Figure 15: MultiSine track of the most dominant signal component in the light curve of the sample project output (8.59 cycles per day), according to the output file output/m000001.dat. Left: amplitude vs. frequency. Mid: phase vs. frequency. Right: amplitude vs. phase. 


\subsection{MultiSine profiles}

A closer examination of the accuracy of the MultiSine fitting procedure is provided by the MultiSine profiles. If the user specifies the keyword msprofs in the .ini file, SigSpec produces additional output files f\#iteration\#.dat, a\#iteration\#.dat, and p\#iteration\#.dat. The idea is to evaluate the rms residual through modifying a single parameter of a single signal component, keeping all other parameters constant. Performing this operation for the frequency of each component produces a set of rms-residual-vs.-frequency plots, all written to the file f\#iteration\#.dat. Correspondingly, the files a\#iteration\#. dat and p\#iteration\#. dat contain rms-residual-vs.-amplitude and rms-residual-vs.-phase plots. The frequencies are scanned around the best fit by $\pm \frac{1}{T \sqrt{\text { sig }}}, T$ denoting the time interval width of the input time series, and sig referring to the signal component under consideration. The amplitudes are calculated from zero to twice the amplitude of best fit, and the phases in a range of $\pm \pi$ around the phase of best fit.

The keyword msprofs is followed by three integer values, the first denoting the number of data points an individual MultiSine profile shall consist of. ${ }^{5}$ The second parameter defines the number of iterations for which MultiSine profiles shall be generated. A negative number causes SigSPEC to generate profiles for all iterations. The third parameter has to be a positive number and defines a step width. If it is set 1 , a MultiSine profile is generated after each iteration, if it is set 2, after every second iteration (starting with $\mathrm{f} 000002$. dat, a000002. dat, p000002.dat), and so on

The output files consist of seven columns:

1. frequency, amplitude, or phase, respectively,

2. rms residual,

3. first-order approximation, based on the tangential gradient (which should be zero, so that the deviation from zero is a measure of the accuracy of the MultiSine fitting procedure),

4. second-order approximation, based on the first and second derivatives at the parameter value of best fit,

5. point-to-point scatter,

6. index of the signal component,

\footnotetext{
${ }^{5}$ Due to the internal accuracy of the index computation, the actual number of points may differ from this value by \pm 1 .
} 
7. index of the harmonic (0 for fundamental), see "Analysis of Harmonics", p. 51.

For each signal component, the first row refers to the parameter value of best fit, as used in the result file.

Example. The sample project output uses the keyword msprofs in the file output.ini, namely

msprofs 10000503

providing MultiSine profiles (f000003.dat, a000003.dat, p000003.dat), (f000006.dat, a000006.dat, p000006. dat),..., for a maximum of 50 iterations of prewhitening sequence. Each MultiSine profile is specified to contain 10000 data points. The number of significant components found in the time series output . dat is 33, so that the last set of MultiSine profiles (f000033. dat, a000033. dat, p000033. dat) refers to the final solution contained in the file result.dat. The MultiSine ptofiles for the primary signal component at 8.59 cycles per day are displayed in Fig. 16.
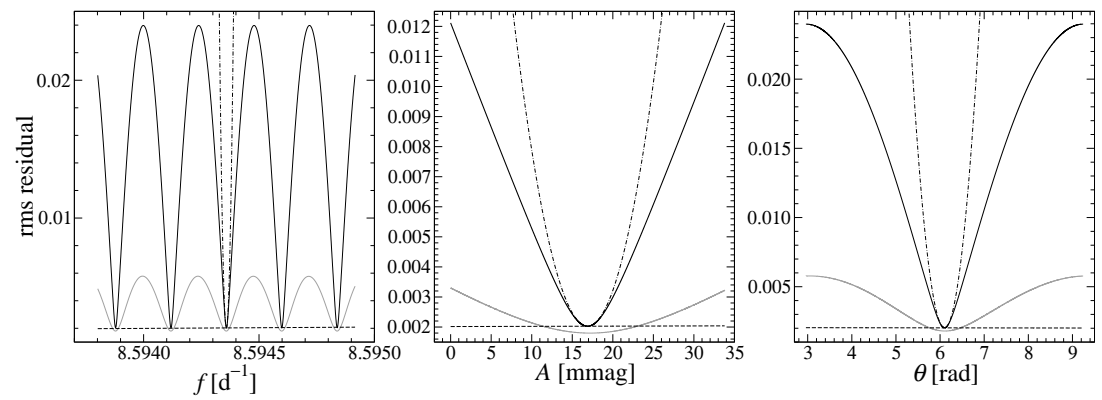

Figure 16: MultiSine profiles of the most dominant signal component in the light curve of the sample project output (8.59 cycles per day), according to the output files output/f000033. dat, output/a000033. dat, output/p000033.dat. Left: rms residual vs. frequency. Mid: rms residual vs. amplitude. Right: rms residual vs. phase. The solid black line refers to the rms residual, the dashed black line to the tangential gradient at the value of best fit (which should be zero), and the dashed-dotted black line to a second-order approximation based on the first two derivatives of rms residual. The solid grey line represents the point-to-point scatter.

\section{Preview}

Since the prewhitening cascade performed by SIGSPEC may be extremely time consuming, the program can compute a preview. This add-on is activated by 
the keyword preview in the .ini file.

Whereas the significance spectra rely on the False-Alarm Probability compared to a noise dataset with the same rms error as the given time series (or series of residuals, respectively), the significance spectrum provided in the file preview. dat represents a set of identified maxima in the significance spectrum of the original time series, but based on the point-to-point scatter in the time domain rather than on the standard deviation of observables. The lower sig limit for writing a local maximum to the file preview. dat is specified as the argument to the keyword preview in the .ini file.

The calculation of the sig is based on the assumption that only the pointto-point scatter is random, and everything else contributing to the rms error represents signal that will be prewhitened in the course of the subsequent loop. The preview output is to be considered as a rough estimate for the final result obtained by step-by-step prewhitening and contains not only the intrinsic variations but also all aliases, which will not occur in the following analysis. The file preview. dat consists of four columns referring to

1. frequency [inverse time units],

2. sig,

3. DFT amplitude [units of observable],

4. phase [rad].

Example. The sample project preview contains a preview file for the $V$ photometry of IC 4996 \# 89. In the file preview.ini, the line

preview:siglimit 5

sets the sig threshold to 5. The output file preview/preview.dat contains 11 components, sorted by frequency. The frequencies and corresponding sigs in the first two columns are

0.9945158494303480

1.9917563998155081

2. 1388902515119841

2. 9835475482878717

3. 1361308018982843

3. 9862375005883859

4. 1333713522847031

4. 9780286490607102

5. 1360613045861099

11.0268647743572874

12.0241053247411784
6.1674140356166323

6. 9302735632389876

6.7175642729893710

8. 6773027802854656

9. 4899187898938777

8. 9589776551282210

8.7492615592402885

5.0523760377159039

5.3438911274207790

5. 5214237212500406

5. 6674302270769710 
Fig. 17 displays the significance and amplitude spectrum of the original time series. Since the preview does not employ any prewhitenings, aliases are present in the file.

- The signal at 3.132 cycles per day corresponds to components \# 3, 5, 7, and 9 .

- The signal at 3.986 cycles per day corresponds to components \# 1, 2, 4, 6 , and 8.

- The signal at 5.409 cycles per day is not found in the preview. In the result of the prewhitening sequence, its sig is 5.02. Since the sig in the preview relies on the rms deviation of the original time series, whereas the final sig is based on the rms deviation after the previous prewhitening step, the sig associated to this frequency falls below the pre-selected threshold of 5 in the preview. The significance spectrum (grey line in the left panel of Fig. 17) shows a peak at the frequency under consideration the sig of which is $\approx 4.8$.

- Components \# 10 and 11 are 1-cycle-per-day aliases of each other, but do not show up in the final result, preview/result. dat.

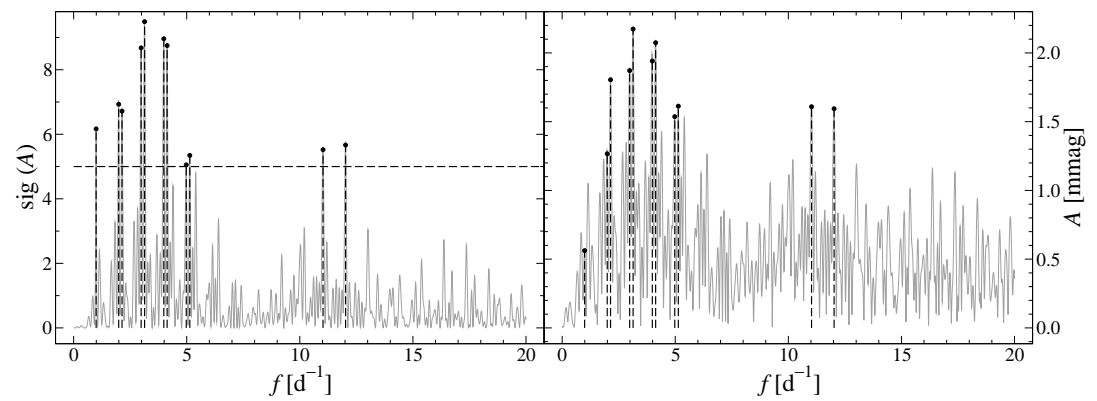

Figure 17: Grey: Fourier spectra for the sample project preview. Left: significance spectrum. Right: DFT amplitudes. The significant components in the preview are indicated by dots with dashed drop lines (file preview/preview.dat). The default sig threshold of 5 is represented by a horizontal dashed line in the left panel.

\section{Correlograms}

SIGSPEC is able to compute correlograms of the time series for each stage of prewhitening. The correlogram files are named c\#iteration\#.dat. The 
calculation of correlograms is activated by the keyword correlograms, which requires three integer parameters. The first parameter represents the maximum order to which to compute serial correlations, i. e. the limit of index lag for each correlogram. Setting it zero forces SigSpec to adjust it to half the number of data points in the time series. The second parameter is the maximum number of iterations for which to compute correlograms. If the number of prewhitening iterations exceeds this value, then no correlogram is generated for the iterations after this limit. If a number $\leq 0$ is given, then a correlogram is computed for each prewhitening stage. The third parameter has to be a positive number and defines a step width. If it is set 1 , a file is generated after each iteration, if it is set 2, after every second iteration (starting with c000002. dat), and so on. The correlogram computation is switched off by default.

A file rescorr. dat is generated, if the keyword correlograms is specified, no matter which parameter constellation is chosen.

A correlogram file consists of two columns referring to

1. index lag,

2. serial correlation coefficient.

Example. The sample project correlograms illustrates how correlograms are generated with SIGSPEC using the V photometry of IC 4996 \# 89 as time series input file correlograms. dat. The file correlograms. ini contains the line correlograms $100-11$

which forces SigSPEC to evaluate correlograms with a maximum index lag of 100 (first parameter) for all iterations (negative value of second parameter). After each iteration, a correlogram is generated (third parameter). The output files

correlograms/c000000. dat correlograms/c000001. dat correlograms/c000002. dat correlograms/rescorr.dat

are generated as displayed in Fig. 18.

\section{Time-resolved Analysis}

In time-resolved mode, SigSPEC performs an analysis for a set of time intervals rather than for the entire time series. An interval of width given by the keyword timeres:range is moved in steps the width of which is given by the keyword timeres: step from the beginning of the time series to the end. ${ }^{6}$ Consecutive

\footnotetext{
${ }^{6}$ In general, the step width is slightly modified by the software to achieve time-resolved analysis over the entire time series.
} 


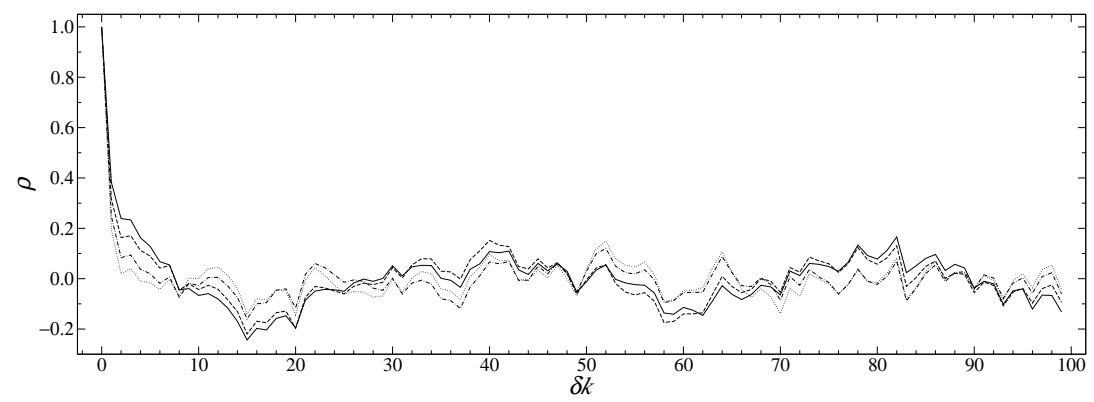

Figure 18: Correlograms for the sample project correlograms. Solid: correlogram of the initial time series (file correlograms/c000000.dat). Dashed: correlogram after one prewhitening (file correlograms/c000001.dat). Dashed-dotted: correlogram after two prewhitenings (file correlograms/c000002.dat). Dotted: residual correlogram after three prewhitenings (file correlograms/rescorr.dat).

time intervals are free to overlap. Time series data within such an interval are used to form a subset for which the analysis is performed. In addition, statistical weights may be applied to the subset data, all with respect to the centre of the interval, which shall be denoted $t_{C}$.

The only exception is the keyword timeres:w:damp. In this case, the analysis is optimised for signal excited at the beginning of the time interval corresponding to the subset under consideration, $t_{B}$ and exponentially damped towards the end of the subset.

The weight functions of time are given in Table 1 . The normalisation of weights is automatically performed by SIGSPEC. Also the combination of a weight function for time-resolved mode with weights columns (keyword col:weights) is supported.

In time-resolved mode, the set of output files as given in "Default Output", p. 24, is generated for each subset of the time series. This requires the introduction of an additional six-digit index, \#interval\#, in addition to \#iteration\#, and the annotation for the output files is

1. wts.\#interval\#. dat for the weight function vs. time in each subset,

2. s\#iteration\#.\#interval\#.dat for the spectra,

3. t\#iteration\#.\#interval\#.dat for the residuals after each step of prewhitening,

4. r\#iteration\#.\#interval\#.dat for the results after each step of prewhitening, 


\begin{tabular}{lll} 
keyword & arguments & weight function \\
\hline timeres:w:none & & 1 \\
timeres:w:ipow & $\xi$ & 0 if $t=t_{C},\left|t-t_{C}\right|^{-\xi}$ else \\
timeres:w:gauss & $\sigma$ & $\mathrm{e}^{-\left(\frac{t-t_{C}}{\sigma}\right)^{2}}$ \\
timeres:w:exp & $\zeta$ & $\mathrm{e}^{-\frac{\left|t-t_{C}\right|}{\zeta}}$ \\
timeres:w:damp & $\zeta$ & $\mathrm{e}^{-\frac{t-t_{B}}{\zeta}}$ \\
timeres:w: $\cos$ & $\nu, \Phi$ & $\cos \left(2 \pi \nu\left|t-t_{C}\right|-\Phi\right)$ \\
timeres:w: $\operatorname{cosp}$ & $\nu, \Phi, \xi$ & $\cos \xi\left(2 \pi \nu\left|t-t_{C}\right|-\Phi\right)$ \\
\hline
\end{tabular}

Table 1: Weight functions for time-resolved SIGSPEC analysis. The beginning of the time interval associated with the referring subset is denoted $t_{B}$, whereas $t_{C}$ symbolises the centre of the time interval.

5. m\#index\#.\#interval\#.dat for the results after each step of prewhitening,

6. result.\#interval\#.dat for the result files, each with a list of significant signal components,

7. residuals.\#interval\#.dat for the final residuals after the prewhitening of all significant signal components,

8. resspec.\#interval\# . dat for the residual spectrum after the prewhitening of all significant signal components,

The column syntax is strictly consistent with the time-unresolved versions (see "Default Output", p. 24). The additional files, wts.\#interval\#.dat, are in two-column format. The first column represents the time values in the corresponding subset, the second column contains the weight function without normalisation.

Furthermore, SIGSPEC generates a file t000000.\#interval\#.dat, which contains the part of the original time series which is actually used as input.

Special functions - as introduced in "Analysis of the Time-domain Sampling" (p. 29), "Preview" (p. 39), and "Correlograms" (p. 41) - are also supplied with the \#interval\# index, i.e.

1. win.\#interval\#.dat for the amplitude windows,

2. profile.\#interval\#.dat for the sampling profiles,

3. sock.\#interval\#.dat for the Sock Diagrams, 
4. phdist.\#interval\#. dat for the phase distribution diagrams,

5. preview.\#interval\#.dat for the previews,

6. c\#iteration\#.\#interval\#.dat for the correlograms after each step of prewhitening,

7. rescorr.\#interval\#. dat for the final correlograms after the prewhitening of all significant signal components.
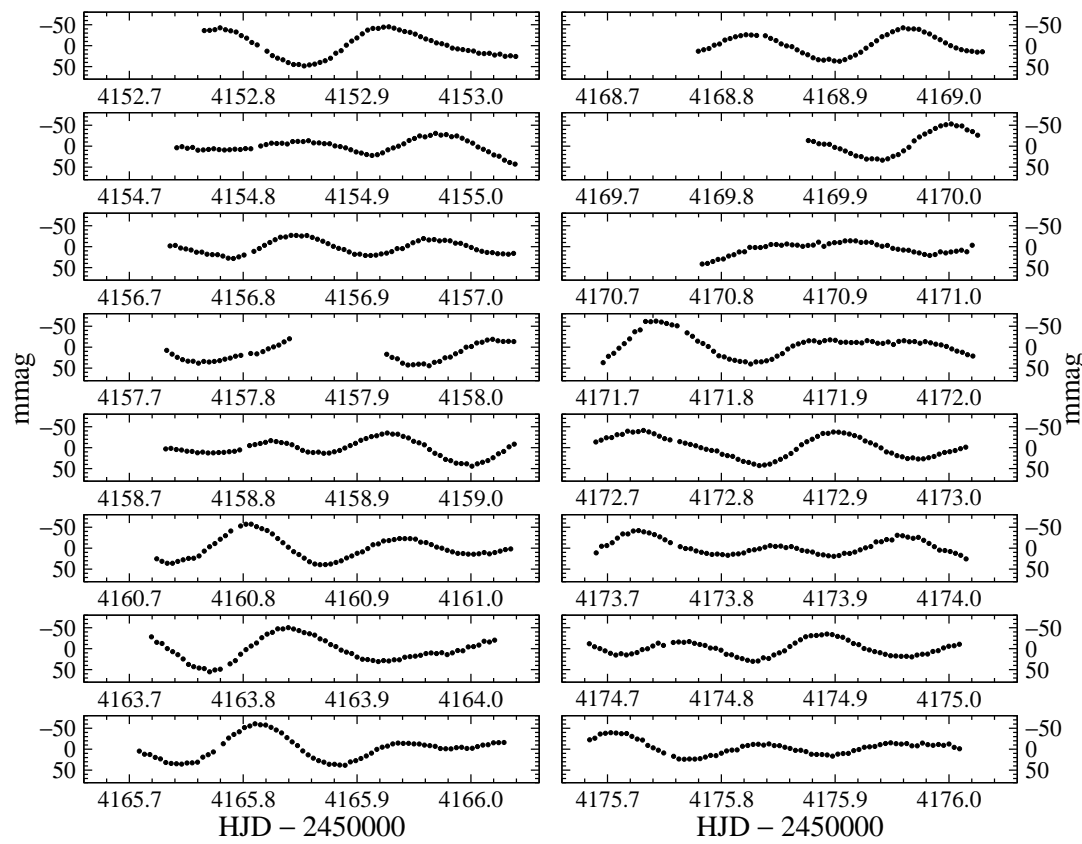

Figure 19: Time series used for the sample project timeres, representing 14 nights of Strømgren $y$ photometry of the Delta Scuti star $4 \mathrm{CVn}$, acquired in February and March, 2007.

Example. The sample project timeres illustrates the time-resolved analysis using Strømgren y photometry of the Delta Scuti star 4 CVn acquired with the Vienna University Automatic Photoelectric Telescope (Strassmeier et al. 1997). The data represent 16 nights from February 21 to March 16, 2007, and are displayed in Fig. 19. 


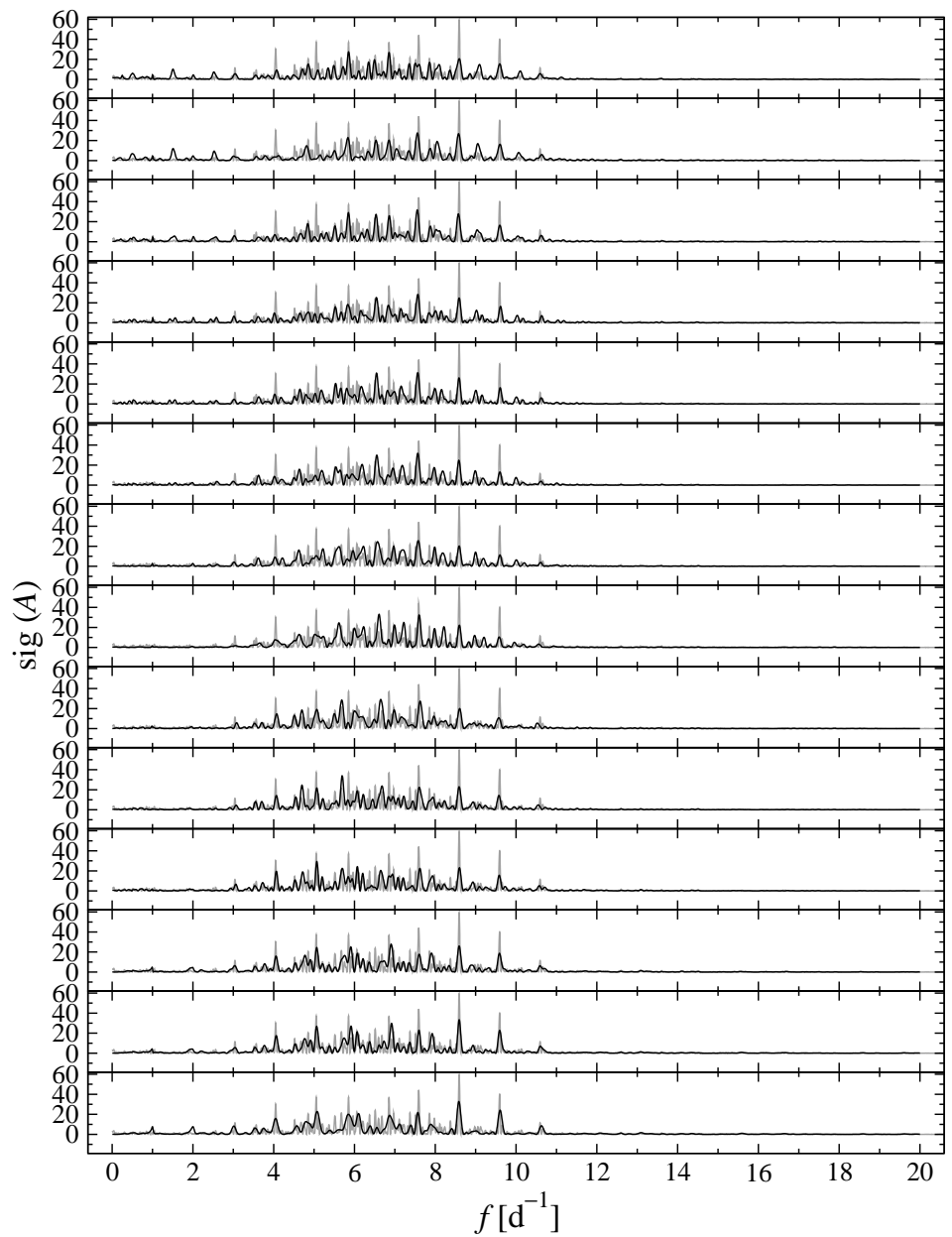

Figure 20: Time-resolved significance spectra for 14 subsets (from top to bottom) automatically generated in the sample project timeres. in each panel, the significance spectrum of the full dataset is displayed in grey colour for comparison.

The file timeres. ini contains the specifications

timeres:range 10

timeres:step 1

which provide a 10-day interval moving over the time base of 24 days, with oneday steps. The resulting 14 subsets are represented by the files 
timeres/t000000.000000. dat to timeres/t000000.000013.dat. Gaussian weight functions with a standard deviation of 5 days are applied:

timeres:w:gauss 5

The files timeres/wts.000000. dat to timeres/wts.000013. dat contain the weights applied to each datapoint within each subset. Further output files are

- timeres/s000000.\#\#\#\#\#.dat for the significance spectra of the original time series without prewhitening (Fig. 20),

- timeres/result.\#\#\#\#\#\#.dat for the lists of significant signal components,

- timeres/residuals.\#\#\#\#\#.dat for the residual time series after all prewhitening steps (divided into subsets according to the time intervals), and

- timeres/resspec.\#\#\#\#\#. dat for the significance spectra of residuals.

Here \#\#\#\#\# denotes six-digit numbers ranging from 000000 to 000013.

\section{SigSpec AntiAIC: Anti-aliasing Correction Mode}

In AntiAIC mode, SigSPEC does not follow a strict step-by-step prewhitening sequence. Instead, test runs are performed for a number of candidate peaks in the significance spectrum in order to find the solution that produces a minimum residual rms scatter after a user-given number of prewhitenings.

1. All peaks above a given sig limit are taken into consideration. The keyword antialc: par in the .ini file is followed by a floating-point number. This quantity is the AntiAIC parameter $p_{\mathrm{al}}$, which has to attain a value in the interval $] 0,1]$. If the highest sig in the considered frequency range is $\max [\operatorname{sig}(A)]$, then the sig limit is $p_{\mathrm{al}} \max [\operatorname{sig}(A)]$. I. e., the AntiAIC parameter determines the sig limit for the candidate peak selection relative to the highest peak in the spectrum under consideration. Alternatively or in addition, a sig threshold for the AntiAIC candidate selection may be defined using the keyword antialc:siglimit. If neither antialc:par nor antialc:siglimit are present, the sig limit specified by siglimit in the .ini file (p. 23) is used for the AntiAIC candidate selection also.

2. The candidate selection is performed for each step in the test prewhitening sequence. 
3. The resulting procedure is the computation of all combinations of candidate peaks above a sig threshold determined by the AntiAIC parameter. The number of iterations for these test prewhitenings is determined by the keyword antialc:depth, followed by an integer value. It specifies the depth of the AntiAIC computation.

4. The successful combination of peaks is selected upon the minimum residual rms deviation out of all examined combinations.

5. SigSpec does not necessarily adopt all iterations performed in the test run for the main prewhitening cascade. The integer value following the keyword antialc:adopt determines how many prewhitening steps shall be adopted. This quantity must not exceed the computation depth provided by the keyword antialc:depth. If the limits specified by the keywords iterations, siglimit, or csiglimit are reached, the output may even terminate before the number specified by the keyword antialc:adopt.

According to Reegen (2007), the expected sig is approximately proportional to the squared amplitude, if all influences by the time-domain sampling are neglected. The combination of $n$ sinusoidal signal components interacting via aliasing is expected to produce a maximum amplitude that does not exceed the sum of amplitudes of the sinusoidal components. Consequently, the square root of the sig of such a combination, sig $_{\text {al }}$, is very likely below the sum of square roots of individual sigs sig ${ }_{n}$,

$$
\sqrt{\operatorname{sig}_{\mathrm{al}}}<\sum_{n} \sqrt{\operatorname{sig}_{n}} .
$$

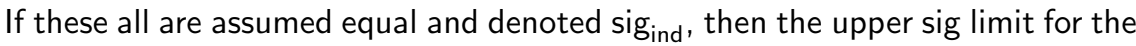
alias is sig $\operatorname{sind}_{\sqrt{n}}$. In other words, if a given peak with a sig sigal is an alias of a combination of $n$ signal components with unique sigs sig ${ }_{\text {ind }}$, then the individual significances are probably higher than $\frac{\mathrm{sig}_{g_{1}}}{\sqrt{n}}$. In terms of the AntiAIC parameter, one obtains

$$
n \approx \frac{1}{\sqrt{p_{\mathrm{al}}}}
$$

for the approximate number of signal components that can be assigned aliasfree for a given AntiAIC parameter $p_{\mathrm{al}}$. Based on these considerations, SIGSPEC evaluates the AntiAIC computation depth using the AntiAIC parameter, if the keyword antialc: depth is not provided in the .ini file.

The AntiAIC mode produces additional screen output, if a combination of candidate peaks yields a lower residual scatter than the previous minimum, a two-line screen message is returned. The first line is a set of indices. In the 
example below, the AntiAIC parameter (keyword antialc:par) is set 0.5, and the AntiAIC computation depth (keyword antialc:depth) is 3. Correspondingly, the first line of output applies to the first of altogether ten candidate peaks in the first iteration, the first out of three in the second iteration, and the first out of seven in the third iteration. This peak constellation produces an rms deviation of residuals as displayed in the second line of output (in the example 0.00405851 ). After finishing the test cascade, the number of iterations specified by the keyword antialc:adopt (in the present example, this number is 2) is adopted for the main cascade. The screen output produced by the main cascade is the same as for a normal SigSPEC prewhitening cascade without AntiAIC. The files containing spectra and residuals, respectively, are updated each time the residual rms deviation improves.
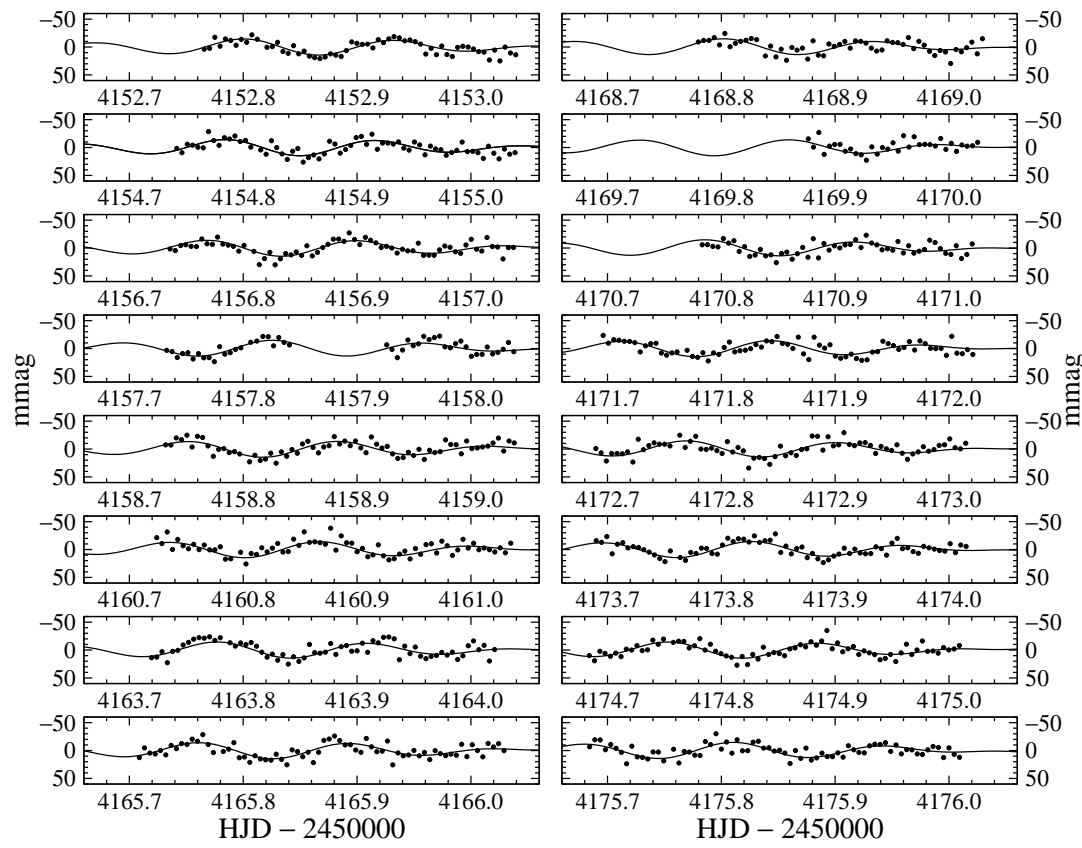

Figure 21: Time series used for the sample project antialc (dots). The sampling represents 14 nights of Strømgren $y$ photometry of the $\delta$ Sct star $4 \mathrm{CVn}$, acquired in February and March, 2007. The magnitude values are synthesized forming two sinusoidal signals (solid line) plus Gaussian noise. 
Example. ${ }^{7}$ The sample project antialc illustrates the anti-aliasing correction using the same sampling as the data for the sample project timeres (p.45),

1. a sinusoid with frequency 6.5598 cycles per day, amplitude 7.29 mmag,

2. a sinusoid with frequency 8.5637 cycles per day, amplitude $6.87 \mathrm{mmag}$,

3. Gaussian noise with 7.36 mmag rms deviation,

as displayed in Fig. 21 . The two signal frequencies differ by almost exactly 2 cycles per day and may easily be misidentified as aliases of each other. There are two identical versions of the light curve provided for comparison: alc.dat and antialc.dat.

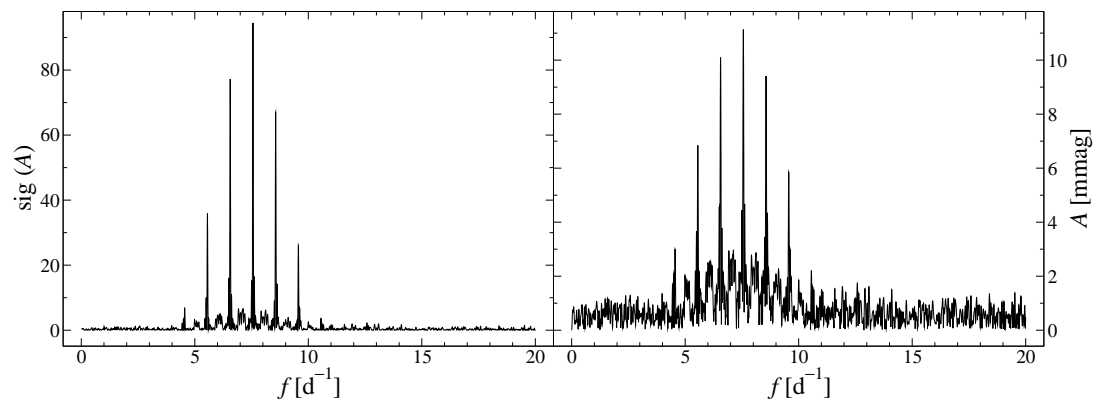

Figure 22: Fourier spectra for the sample project antialc. Left: significance spectrum. Right: DFT amplitudes.

The file alc.dat corresponds to the project directory alc, representing a normal SigSpec run without a file alc.ini. Running SigSpec alc, the resulting frequencies (screen output) are

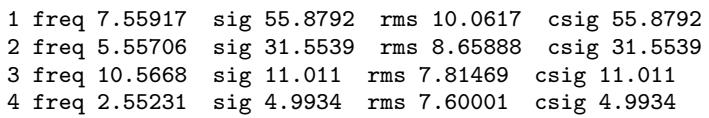

Instead of the two signal components, 1-cycle-per-day aliases are identified. The significance and Fourier amplitude spectra of the dataset show the highest peak at 7.56 cycles per day, which represents a superposition of the first upper side peak of the signal at 6.56 cycles per day and the first lower side peak of the signal at 8.56 cycles per day (Fig. 22). This leads to an imperfect prewhitening of the two components, and the remaining signal is detected as a third component at 9.56 cycles per day.

\footnotetext{
${ }^{7}$ The computation of the sample project antialc takes 7 minutes on an Intel Core2 CPU T5500 $(1.66 \mathrm{GHz})$ under Linux 2.6.18.8-0.9-default i686.
} 
The alternative AntiAlC analysis is provided by the file antialc.ini, which contains the specifications

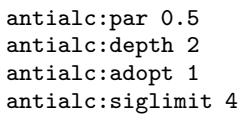

All peaks that reach at least $50 \%$ of the highest significance in the spectrum are taken into account. SIGSPEC computes two consecutive iterations, but adopts only the first of these two iterations. A sig limit of 4 is assumed for the AntiAIC calculations (contrary to the default sig limit of 5 still valid as a breakup condition for the whole procedure). Running SigSpec antialc, the screen output is

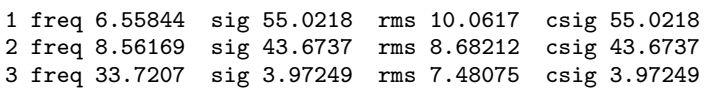

Both signals are recovered at a reasonable frequency accuracy. Moreover, according to the file antialc/result. dat, the amplitudes of the two signals are recovered to a satisfactory precision (7.22 $\mathrm{mmag}, 6.47 \mathrm{mmag}$ ).

\section{Analysis of Harmonics}

If a non-sinusoidal, but periodic process is measured, DFT does not only produce the fundamental frequency, which is the repetition rate of the non-sinusoid. The shape of the periodicity is recovered by a number of harmonics (also called overtones) the frequencies of which are integer multiples of the fundamental. In this case it may be considered insufficient to determine the exact frequency of the process by employing only the peak at the fundamental frequency and ignoring the harmonics. The keyword harmonics, followed by an integer determining the upper limit of the harmonic order, allows to compute the sig of the fundamental plus the desired number of overtones. The specification harmonics 20 forces SIGSPEC to take into account altogether 21 frequencies.

As pointed out by Reegen (2007), SIGSPEC treats False-Alarm Probabilities in a statistically clean and unbiased way. In analogy to the comb analysis introduced by Kjeldsen et al. (1995), but benefitting from the exact statistical treatment of noise, it is possible to extend the method in order to evaluate the probability of a whole set of peaks to be generated by noise simultaneously. This strategy helps to take into account a fundamental frequency plus a set of integer multiples at once and permits to evaluate the most likely solution for a non-sinusoidal signal. In addition, the Fourier Space parameters obtained for the signal components provide a fit to the data in terms of a fundamental frequency plus overtones. 
Given a set of amplitude levels $A_{h}, h=0,1, \ldots, H$, at different frequencies with associated False-Alarm Probabilities $\Phi_{\mathrm{FA}}\left(A_{h}\right)$, the probability that all amplitude levels are due to noise is given by the product of the individual False-Alarm Probabilities,

$$
\Phi_{\mathrm{FA}}\left(\bigwedge_{h=0}^{H} A_{h}\right)=\prod_{h=0}^{H} \Phi_{\mathrm{FA}}\left(A_{h}\right),
$$

if the noise amplitudes at the two frequencies are assumed statistically independent. This is the probability that all amplitude levels are generated by noise.

Since the sig is defined as the negative logarithm of False-Alarm Probability, the above expression leads to

$$
\operatorname{sig}\left(\bigwedge_{h=0}^{H} A_{h}\right)=\sum_{h=0}^{H} \operatorname{sig}\left(A_{h}\right) .
$$

In this context, the sig represents the number of cases in one out of which all amplitude levels $A_{h}$ are not generated by noise. This logical concept is the representation of an AND operator, as indicated by the argument to sig in the equation.

Reegen (2007) evaluated the expected value of the sig (ignoring the variations with frequency and phase) to be $\frac{\pi}{4} \log \mathrm{e} \approx 5.4575$. Considering $H$ different amplitude levels simultaneously rescales this expected sig, so that we obtain $\frac{H \pi}{4} \log$ e. This rescaling may cause inconvenience, whence we use the mean sig of an individual peak out of this sample of fundamental plus harmonics,

$$
\operatorname{msig}\left(A_{h}\right):=\frac{1}{H+1} \operatorname{sig}\left(\bigwedge_{h=0}^{H} A_{h}\right),
$$

instead. It is the expected sig obtained for an arbitrarily picked element out of the $H$ peaks: if each of the considered peaks would have msig $\left(A_{h}\right)$, then the total sig of the fundamental plus harmonics would be $\operatorname{sig}\left(\bigwedge_{h=0}^{H} A_{h}\right)$. The statistical properties of msig $\left(A_{h}\right)$ are the same as for the "normal" sig analysis. If the keyword harmonics is provided in the .ini file, the sig levels returned in the second column of the file result. dat are mean sigs.

The result files display only the fundamentals of the solution, and information on the harmonics is stored in additional output files. The names are generated from the name of the corresponding result file without the extension .dat, plus -h\#index\#.dat, where \#index\# refers to the index of the item in the result file. For example, the harmonics for the third component in the file result. dat are stored in the file result-h000003. dat. The files contain 
the harmonics in ascending order, starting with the fundamental. The three columns are

1. sig of the individual peak,

2. DFT amplitude [units of observable],

3. Fourier-space phase angle [rad].

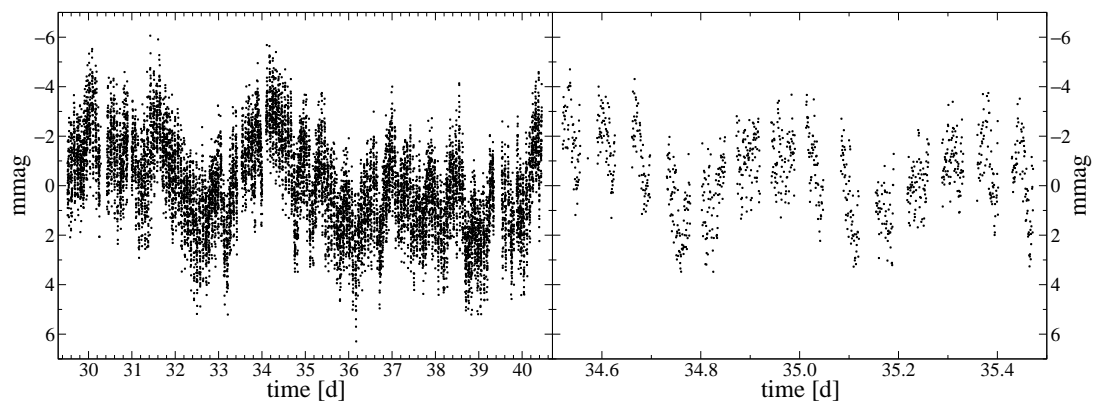

Figure 23: Time series used for the sample project harmonics.

Example. The sample project harmonics illustrates the determination of a non-sinusoidal signal using the analysis of harmonics. The dataset represents (yet unpublished) space photometry of a star that exhibits surface activity. The task is to determine the rotation period of the star. For comparison, two identical versions of the time series are avalable (Fig. 23). The file noharmonics. dat is used together with the file noharmonics.ini to perform a SIGSPEC analysis without harmonics and associated with the project directory noharmonics containing the output. It contains four lines:

ufreq 13

freqspacing .001

iterations 1

siglimit 0

In this constellation, SIGSPEC computes the significance spectrum between 0 and 13 cycles per day, with steps of 0.001 cycles per day (Fig. 24, left panel). Only one iteration (i.e. no prewhitening) is performed. The highest peak is found at 0.296 cycles per day, which corresponds to a period of 3.38 days. 


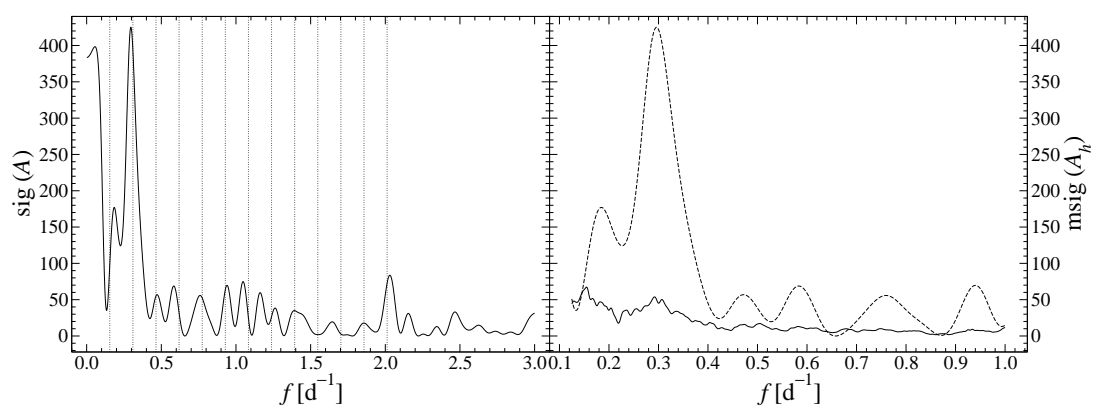

Figure 24: Fourier spectra for the sample project harmonics. Left: significance spectrum without employing the analysis of harmonics (solid line). The fundamental and twelve harmonics of the alternative solution are indicated by vertical dashed lines for comparison. Right: significance spectrum displaying the mean sig for fundamental plus twelve harmonics (solid line). Note that the frequency interval differs from the left panel. For comparison, the solution without harmonics is displayed as a dashed line.

The file harmonics. dat is the same as noharmonics.dat, but the associated file harmonics. ini specifies a different setup by the lines

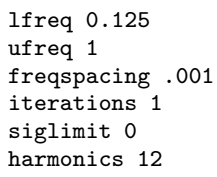

It is advisable not to set the lower frequency limit zero, because below the Rayleigh frequency resolution, consecutive harmonics hit the same peak and produce unreliable results. In the present case, the Rayleigh frequency resolution is 0.091 cycles per day, and to be fairly on the safe side, the lower frequency limit is adjusted to 0.125 cycles per day. Fig. 24(right panel) contains the mean sig of the fundamental plus twelve harmonics vs. frequency.

The amplitudes of the fundamental and twelve harmonics are displayed vs. frequency in Fig. 25. The maximum sig is found at 0.155 cycles per day, i. e., the rotation period is 6.46 days, indicating that the analysis without harmonics led to a misidentification of the first harmonic as the "true" rotational frequency. For comparison, the left panel of Fig. 24 contains the fundamental plus harmonics found by this procedure as vertical dashed lines.

Moreover, for the analysis of harmonics, there is additional information in the screen output provided by SIGSPEC. The standard screen output for the project noharmonics contains the lines 


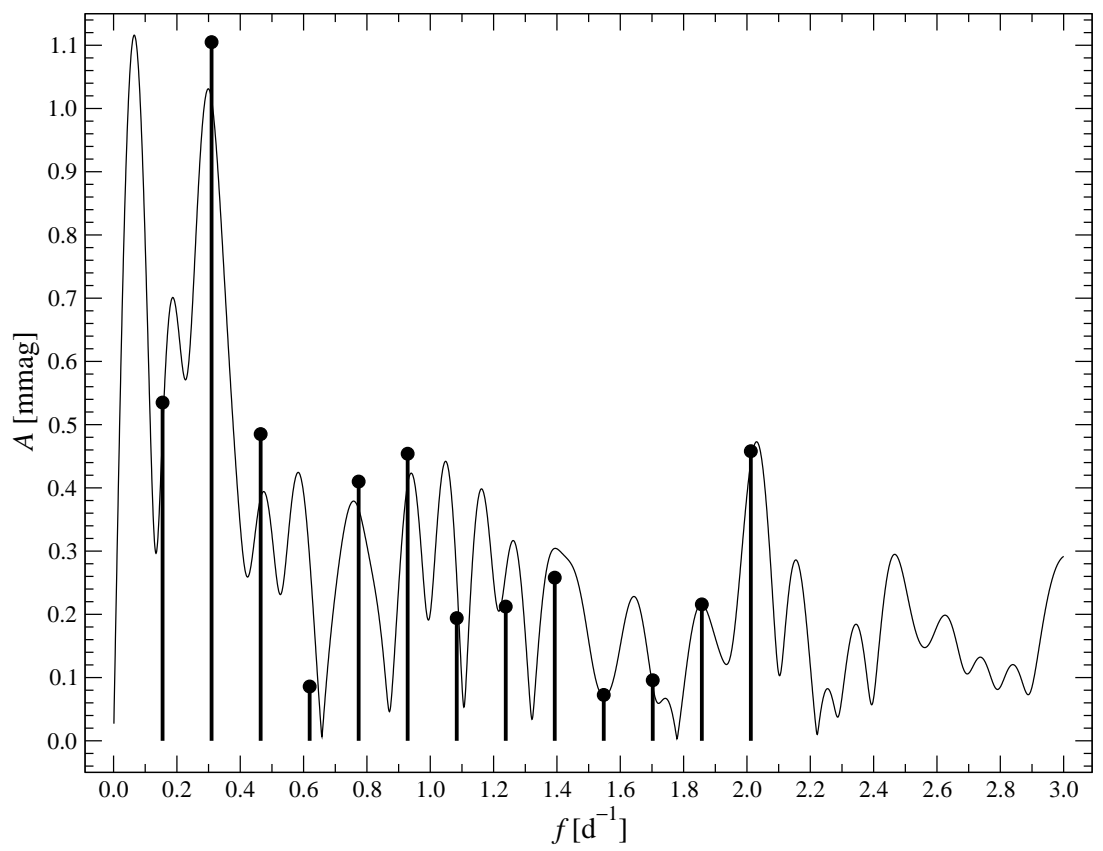

Figure 25: Frequencies and amplitudes of the harmonics associated to the most significant signal found for the sample project harmonics (dots with drop lines). The DFT amplitudes obtained by SIGSPEC without employing the analysis of harmonics are displayed as a solid line for comparison.

preparing to run SigSpec

Rayleigh frequency resolution

oversampling ratio

frequency spacing

lower frequency limit

upper frequency limit

Nyquist coefficient

number of frequencies
0.0914470160931467

91.4470160931467433

0.0010000000000000

0.0010000000000000

13.0000000000000000 13000

0.9993990384615384

For the project harmonics, the corresponding output is richer.

*** preparing to run SigSpec

Rayleigh frequency resolution

0.0914470160931467

oversampling ratio

91.4470160931467433

frequency spacing

0.0010000000000000

lower frequency limit

0.1250000000000000

upper frequency limit

Nyquist coefficient

number of frequencies

13.0000000000000000 12876 
upper fundamental frequency number of fundamental frequencies
1.0000000000000000

876

Although the upper frequency limit is set 1 cycle per day by the keyword ufreq. SIGSPEC has to compute the Fourier spectrum up to a frequency of 13 cycles per day in order to cover also the 12 harmonics. Two additional lines are provided corresponding to the upper limit for the fundamental frequencies, which is related to the specification by ufreq in the file harmonics.ini, and the number of fundamental frequencies.

\section{MultiFile Mode}

12.1. How to handle multiple time series

An additional feature of SIGSPEC is the ability to handle multiple time series input files at once. This increases the performance of the program significantly, if the time values in all input files are identical.

- The user has to provide only one project directory <project $>$ - just as in SingleFile mode (as described in "Projects", p. 7).

- Parameter specifications in the file 〈project $\rangle$. ini are uniquely applied to all time series input files. Thus SIGSPEC expects the same column format for all time series input files and applies the settings specified in the . ini file to all input files.

- Time series files have to be indexed as \#multifile\#.<project>.dat, where \#multifile\# represents a six-digit index starting with 000000 . Note that strictly ascending indices are required.

- All output files are supplied with the leading index \#multifile\#. For example, 000012.s000009.000002 denotes the significance specturm the second iteration for time interval number nine in a time-resolved analysis of the 12 th file in MultiFile mode.

- The MultiFile mode is activated by the keyword multifile, followed by an integer value. This value is interpreted as the maximum index up to which the calculations shall be performed. This permits a restriction for, e. g., test runs. If the index limit is assigned a negative value, SIGSPEC analyses as many files as available.

- The sampling profile of the file 000000 . <project $>$. dat is always written to a file. For subsequent and consistent time series, the sampling profile is taken from this file, which saves computation time. Only if the new 
time values are inconsistent with those of the precursor, the profile is re-calculated and stored in a corresponding output file for later use. The keyword profile in the ini file is ignored in MultiFile mode.

In MultiFile mode, SigSpec terminates, if a \#multifile\# index is reached, for which no time series input file is available.

A further keyword to restrict the MultiFile analysis is mfstart, which permits to specify a MultiFile index to start with (instead of 0 ).

Example. The two lines

mfstart 4

multifile 16

activate the MultiFile mode for input files from $000004 .<$ project $>$. dat to 000016 . project>. dat.

The big advantage of the MultiFile mode is that sampling profiles are computed only if necessary. If the time-domain sampling is identical to a previously examined time series, the sampling profile of this time series is used. If the keyword profile is set in the .ini file, a file assign. log is generated. It contains a table of assignments between time series file indices and profile indices.

Example. The line

000013

$000002\}$

in the file assign. $\log$ means that for $000013 .\langle$ project $>$. dat, the profile with index 000002 is used.

Example. The sample project multifile illustrates the simultaneous analysis of multiple time series. The project contains 10 time series files from 000457.multifile.dat to 000467 .multifile.dat. However, the files do not represent a complete sequence, since 000466. multifile. dat is missing. The lines

mfstart 457

multifile 467

in the file multifile.ini would force SIGSPEC to process the complete sequence of time series input files. Indeed, the program starts with the file 000457.multifile.dat and proceeds until 000465.multifile.dat. Since the next file, 000466.multifile.dat is missing, it stops its calculations with 000465 . multifile. dat and displays a corresponding warning:

Warning: MultiFile_Count 002

MultiFile limit exceeds number of available

time series input files, limit re-adjusted to 465 . 
The keyword profile in the file multifile.ini forces SIGSPEC to generate the following files in the project directory:

000457.profile.dat

000458.profile.dat

000460.profile.dat

000463.profile.dat

The reason why only four profiles are computed for nine time series is found in the file assign. log:

time series input file

000457
000458
000459
000460
000461
000462
000463
000464
000465 profile and spectral window

000457
000458
000457
000460
000457
000457
000463
000457
000457

000457

000460

000457

The contents of this file tell the user that the samplings of all time series files are identical, except for those with indices 000458, 000460 and 000463. In order to speed up the computations, SigSpec generates only one profile for the files with identical sampling and re-uses this profile for all of them. The first file with this sampling is 000457 .multifile.dat, and the associated profile is also used for

If the keyword win is added to the file multifile.ini, this assignment applies to the files containing the spectral windows as well.

\subsection{Differential significance spectra}

Practical astronomical time series analysis occasionally comes along with target and comparison datasets that show coincident peaks in the DFT amplitude spectra. In this case, SIGSPEC provides a possibility to compute the probability that a peak in the target dataset is significant in spite of a given peak in the comparison dataset. Moreover, multiple target and/or comparison datasets may be handled the same way. The idea is to identify common (instrumental and/or environmental) effects and to distinguish them from periodicities exclusively found in a target dataset. 
In the .ini file, there are three different keywords reserved for the specification of dataset types. Each expects one integer parameter representing the Multifile index of the dataset under consideration.

1. The keyword target specifies a target dataset.

2. The keyword comp specifies a comparison dataset.

3. The keyword skip specifies a dataset to be ignored.

To enhance the convenience for the user, not all files need to be specified. The keyword deftype may be used to assign a default dataset type.

1. Use deftype target to assign the target attribute by default. If no deftype keyword is provided, this setting is activated.

2. Use deftype comp to assign the comp attribute by default.

3. Use deftype skip to assign the skip attribute by default.

Sampling profiles need to be computed for target datasets only. If the keyword profile is given in the .ini file, sampling profiles will only be generated for target datasets, and the file assign.log will also contain target datasets only.

To make datasets comparable even if their quality is different, the DFT spectra of the comparison datasets are scaled according to the power integral over the entire frequency range under consideration.

Instead of the observables $c_{k}, k=0,1, \ldots, K$ of a comparison dataset, the transformed quantities

$$
c_{k}^{\prime}:=\frac{P\left(x_{l}\right)}{P\left(c_{k}\right)} c_{k}
$$

are used, where $x_{l}, I=0,1, \ldots, L$ denotes the observables of the target dataset under consideration and $P$ indicates the power integral of the quantity in parentheses. A DFT is calculated for each comparison dataset. There are two options to determine the resulting amplitude $A_{T}$ to be compared to the target amplitude A.

By default, the sig measures the probability of a peak generated by noise at the same variance as that of the given time series. In case of computing differential sigs, the normalisation has to be modified, since part of the power found in the target spectrum is assumed due to corresponding power in a comparison spectrum. To take this into account appropriately, a factor

$$
\gamma:=\frac{P\left(x_{l}\right)}{d P}
$$


is introduced, where $d P$ is the power integral of the difference between the target data and the transformed comparison data. Correspondingly, the differential sig is a measure of the additional power with respect to the comparison dataset to be due to noise.

1. If the keyword diff :comp is set in the .ini file, a weighted arithmetic mean of the Fourier vectors, averaged over all comparison datasets is used to calculate $A_{T}$. The numbers of data points the comparison datasets consist of are used as weights. This option considers signal common among the comparison datasets only if the phases are aligned. Following the formalism by Reegen (2007), the cartesian representation of the differential sig evaluates to

$$
\begin{gathered}
\operatorname{sig}\left(a_{\mathrm{ZM}}, b_{\mathrm{ZM}} \mid \omega\right)=\gamma \frac{K \log \mathrm{e}}{\left\langle x^{2}\right\rangle} \times \\
\left\{\left[\frac{\left(a_{\mathrm{ZM}}-a_{T \mathrm{ZM}}\right) \cos \theta_{0}+\left(b_{\mathrm{ZM}}-b_{T \mathrm{ZM}}\right) \sin \theta_{0}}{\alpha_{0}}\right]^{2}\right. \\
\left.+\left[\frac{\left(a_{\mathrm{ZM}}-a_{T \mathrm{ZM}}\right) \sin \theta_{0}-\left(b_{\mathrm{ZM}}-b_{T \mathrm{ZM}}\right) \cos \theta_{0}}{\beta_{0}}\right]^{2}\right\} .
\end{gathered}
$$

2. If the keyword diff: compalign is set in the .ini file, a weighted arithmetic mean of the DFT amplitudes, averaged over all comparison datasets is considered as $A_{T}$. The numbers of data points the comparison datasets consist of are used as weights. This option considers signal common among the comparison datasets also if they lag in phase. The differential sig is obtained through

$$
\operatorname{sig}\left(A \mid A_{T}\right)=\gamma \frac{K\left(A-A_{T}\right)^{2} \log \mathrm{e}}{4\left\langle x^{2}\right\rangle}\left[\frac{\cos ^{2}\left(\theta-\theta_{0}\right)}{\alpha_{0}^{2}}+\frac{\sin ^{2}\left(\theta-\theta_{0}\right)}{\beta_{0}^{2}}\right] .
$$

following the annotation introduced by Reegen (2007).

The default setting is diff:off, which switches off the computation of differential sigs.

Additional output is provided in the spectra (see p. 26), where columns 6 and 7 contain the DFT amplitudes and phases of the transformed comparison dataset, respectively.

Example. The sample project diffsig illustrates the analysis of target and comparison time series using differential significance spectra. There are nine time series input files available, indexed from 000038 through 000046 . The file diffsig.ini contains the lines 
which forces SIGSPEC to start with the file 000038. diffsig. dat and compute all available datasets. In this case. SIGSPEC takes into account all files from 000038 to 000046 . The two lines

deftype target

comp 38

in the file diffsig. ini define the file 000038.diffsig.dat as a comparison dataset and the rest as targets. Thus differential significance spectra are calculated for all time series from 000039 through 000045 , with respect to 000038 as comparison data. The calculation of differential sigs is activated by the line

diff : compalign

in the file diffsig.ini, which produces differential sigs without respect to phase lags between comparison and target signals. The computations are made faster by the lines

ufreq 7

siglimit 0

iterations 1

The sampling of the input file 000038.diffsig. dat represents the $V$ photometry of IC 4996 \# 89 (see Example SigSpecNative, p. 8), and the observable is a synthetically generated signal with unit amplitude at a frequency of 3.125 cycles per day, plus Gaussian noise with 5 units rms deviation. The corresponding significance spectrum, as obtained by typing

SigSpec 000038.diffsig

is displayed in the bottom panel of Fig. 26. The five upper panels contain the differential significance spectra of the time series 000039 to 000046 . These datasets contain 11649 points and are based on the sampling used in the project harmonics (p.53). Gaussian noise with a standard deviation of 100 units is generated. Just as in case of the comparison data, a sinusoid at 3.125 cycles per day is synthesized, but the phase is not the same as for 000038. diffsig. dat. The amplitudes of this signal are 5 units for 000039, 6 units for 000040,7 units for 000041, 8 units for 000042, 9 units for 000043, 10 units for 000044, 11 units for 000045, and 12 units for 000046 . With increasing signal amplitude in the target data, the differential sig of the main peak consistently increases. In Fig. 26 the datasets 000039 to 000046 are displayed from bottom to top. 


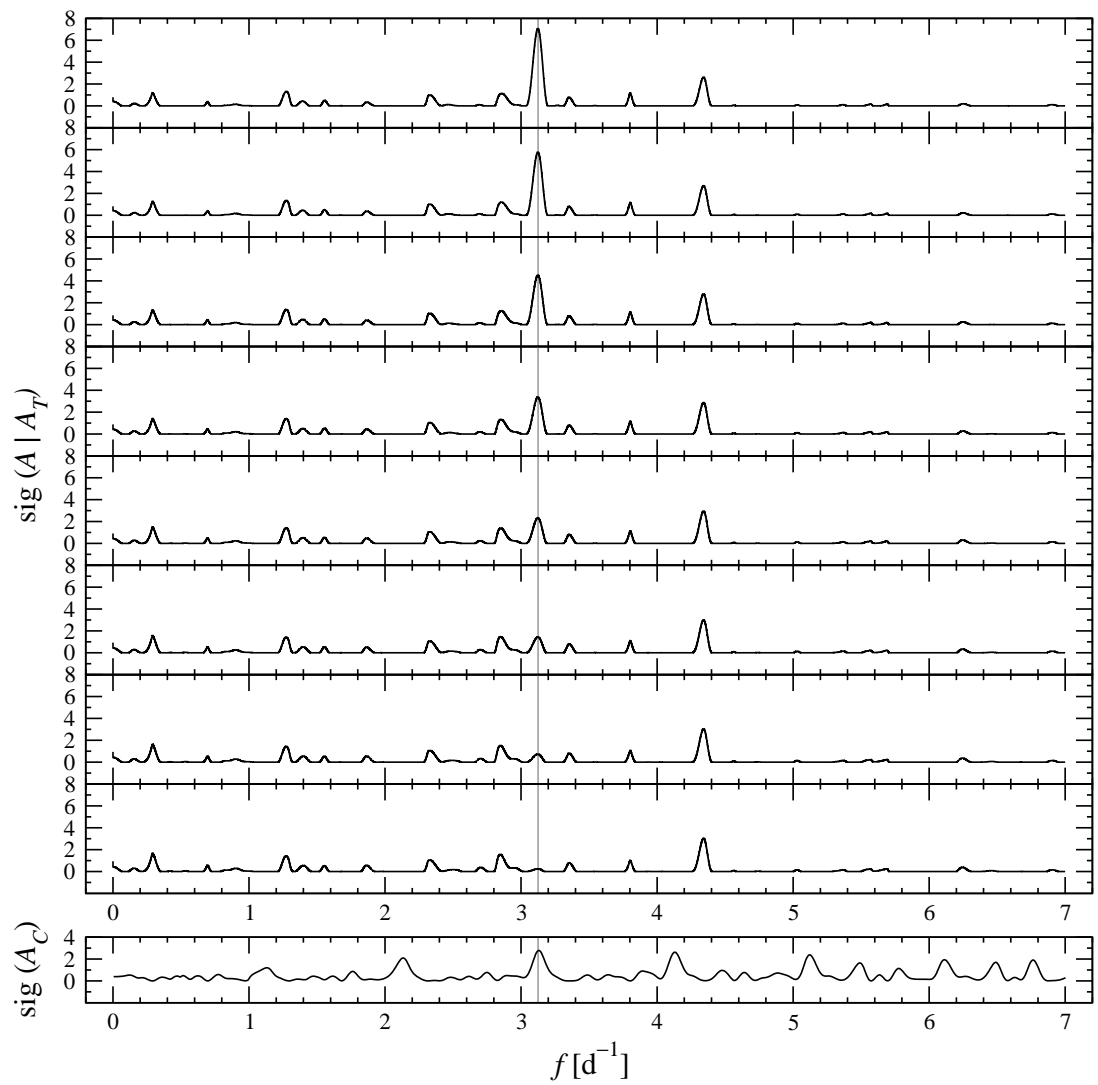

Figure 26: Differential significance spectra for the sample project diffsig. Bottom: significance spectrum of comparison data, representing a sinusoidal signal at 3.125 cycles per day (grey line), plus Gaussian noise. Top eight panels: Differential significance spectra for target time series representing the Gaussian noise plus a sinusoidal signal at 3.125 cycles per day. Both the time-domain sampling and the signal phase differ from the comparison data. From bottom to top, the amplitude of this signal increases.

\section{The Built-in Simulator}

SIGSPEC contains a simulator to generate and analyse synthetic time series. To activate the simulator, a sequence of keywords may be given in the .ini file to generate a variety of datasets. The sampling is taken from the time series input file.

The simulator activities specified by sim:signal, sim:poly, sim:exp, 
sim:serial, sim:temporal, sim:rndsteps, and sim:zeromean are interpreted as a sequence and performed step by step, following their order in the . ini file. SIGSPEC generates the synthetic light curve by performing all specified actions following the order of occurrence in the .ini file.

The synthetic time series is saved as a file with the same name as the input, but in the project directory, to avoid accidential overwriting of original data. If the time series input file is named <project>. dat, then the synthetic time series is <project>/<project>.dat. In MultiFile mode, if the time series input files are named \#multifile\#.<project>.dat, the synthetic time series are $\langle$ project $>/ \#$ multifile\#.<project $\rangle$. dat.

\subsection{The simulator mode}

SIGSPEC supports two different simulator modes.

1. The keyword sim: add runs the simulator in additive mode. The program keeps the original observable values and adds the synthetic values. For example, this function is useful to add synthetic noise to a given time series.

2. The keyword sim:replace forces the simulator to overwrite the original observable values with the synthetic values.

3. The keyword sim: off is used, if no simulator activity is desired. Since the simulator is deactivated by default, this keyword is redundant and only implemented for completeness.

\subsection{Random numbers}

The SIGSPEC simulator is capable of modelling three different types of random processes:

- serially correlated noise (keyword sim:serial, p. 70),

- temporally correlated noise (keyword sim:temporial, p. 72),

- random steps (keyword sim:rndsteps, p. 74.

The random number generator employed for these models may be initialised in two different ways.

1. The user may pass an integer value to the program. This value has to be written into a file <project>.rnd.

2. If the file <project>. rnd is not present, the simulator initialises the random number generator using the system time. 
The last integer value in the sequence of random numbers is written to a file <project>/<project>. rnd. This allows to embed SigSPEC into an external loop for numerical simulations. If the output file $\langle$ project $\rangle /\langle$ project $\rangle$. rnd is moved to <project>. rnd externally between consecutive SIGSPEC runs, the program may used iteratively without breaking the random number sequence.

Example. The simulator is employed in the sample projects sim-serial, sim-temporal and sim-rndsteps. To initialise the random number generator, a file sim-serial.rnd, sim-temporal.rnd and sim-rndsteps.rnd, respectively, is used to make the output reproducible.

Consequently, the user has three options to explore the these samples.

1. If the samples are processed as they are, SIGSPEC reproduces the given output exactly.

2. If the .rnd file in the input directory is removed by the user, SigSpEC produces a new set of random numbers. The random number generator is initialised employing the system time.

3. If the content of the .rnd file in the input directory is modified by the user, SIGSPEC produces a new set of random numbers. The random number generator is initialised employing the new number in the . rnd file.

\subsection{Sinusoidal signal}

The keyword sim:signal is given with five floating-point parameters. They specify

1. the lower time limit,

2. the upper time limit,

3. the amplitude,

4. the time zeropoint (a fixed time where the signal shall attain a maximum), and

5. the frequency [inverse time units].

If the lower and upper time limits are both set zero, the signal is generated for the entire time base.

Example. The sample project sim-signal contains the simulation and analysis of two sinusoidal signals, one over the entire time base, one on a restricted time 

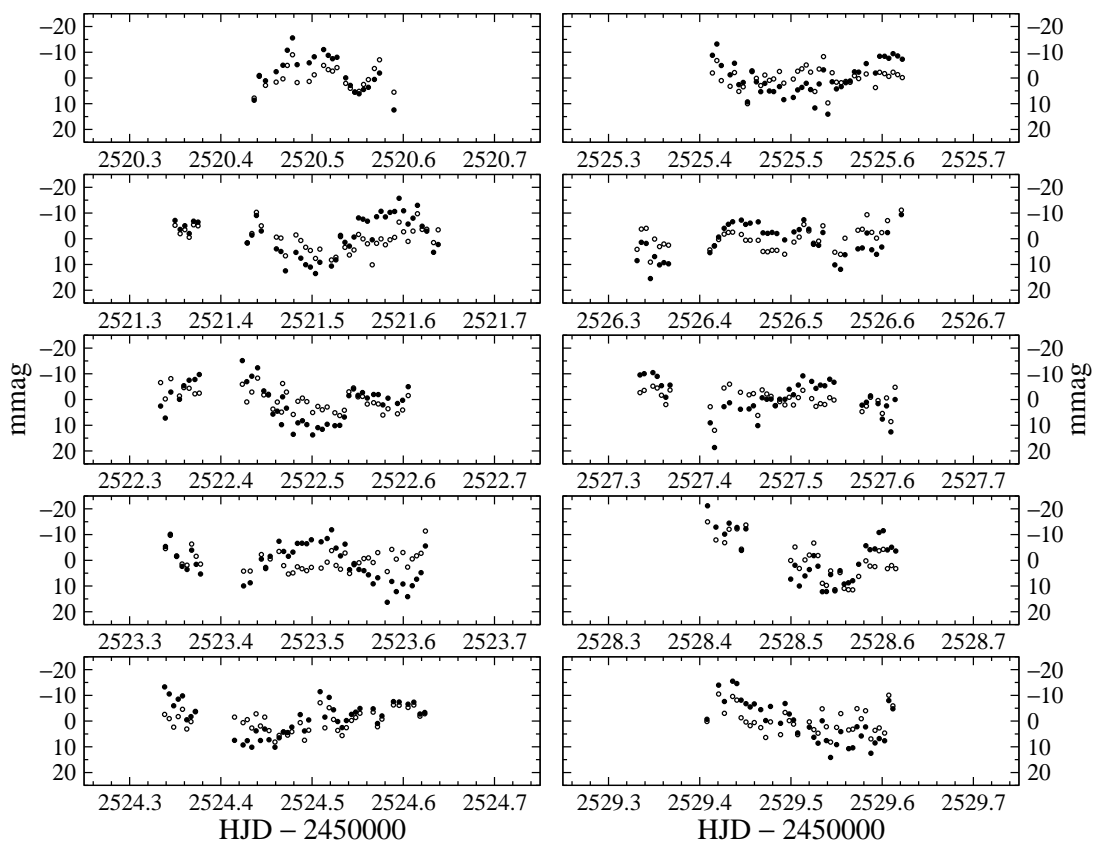

Figure 27: Time series generated by the simulator in the sample project sim-signal. Open circles: Original V photometry of IC 4996 \# 89. Dots: Two sinusoidal signals added by the simulator.

interval. In this sample project, the $V$ photometry of IC 4996 \# 89 (see Example SigSpecNative, p. 8) is modified, according to the line

sim: add

in the file sim-signal.ini. The line

sim:signal $000.00727 \quad 2521.4542 \quad 4.68573$

produces a sinusoidal signal over the entire time base (corresponding to the first two arguments being zero). The amplitude is $7.27 \mathrm{mmag}$, and the frequency is 4.68573 cycles per day. At HJD 2452521.4542 the sinusoid shall attain zero value. Correspondingly, the line

sim:signal $252125250.005432524 .2356 \quad 6.24512$

is associated to a sinusoid with amplitude $5.43 \mathrm{mmag}$, frequency 6.24512 cycles per day, and a zeropoint at HJD 2452524.2356. This signal is not generated 
for the entire time base but only from HJD 2452521 to HJD 2452525. Fig. 27 displays the light curves of the original and the synthetic data.

The screen output contains the lines

*** simulator: add

signal

signal

indicating that the simulator adds the synthetic values to the original observables, and that two sinusoids are generated.

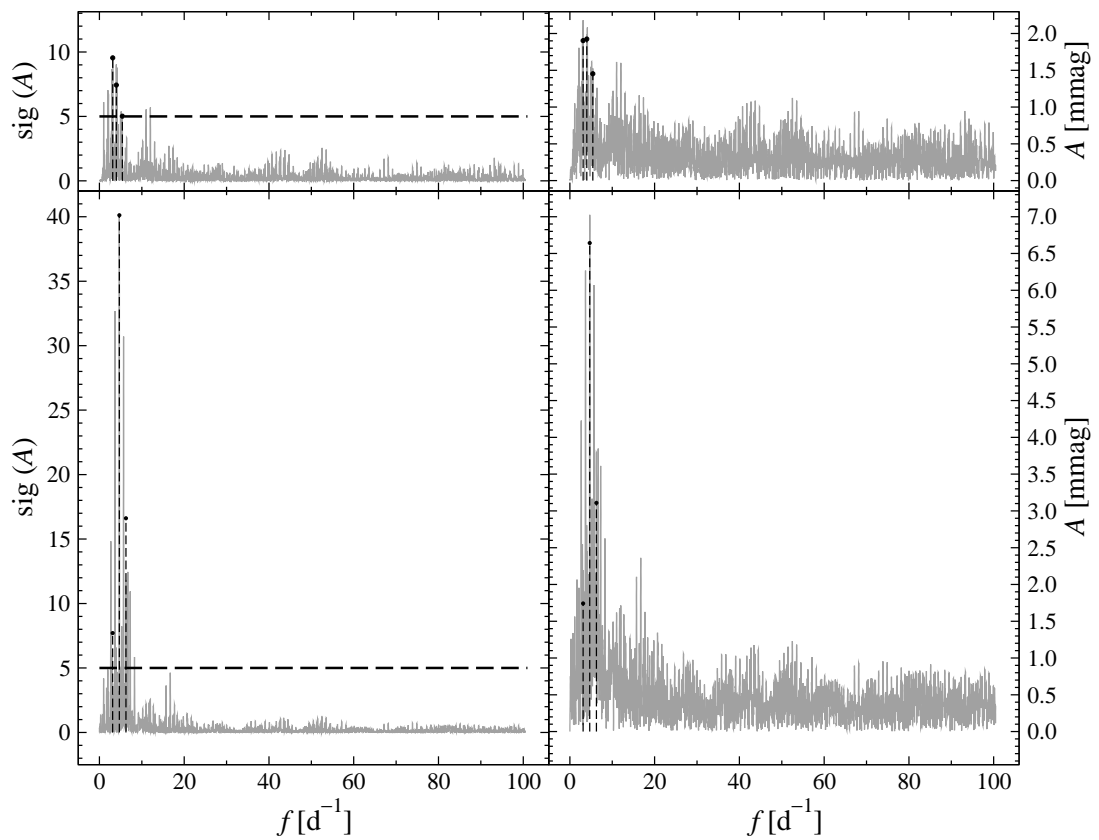

Figure 28: Fourier spectra for the sample project sim-signal. Left: significance spectra. Right: DFT amplitudes. Top: original spectra (file SigSpecNative/s000000.dat). Bottom: spectra with two sinusoidal signals added. All spectra are plotted grey. The significant components are indicated by black dots with dashed drop lines (file SigSpecNative/result.dat for the top panels, file sim-signal/s000000. dat for the bottom panels). The default sig threshold of 5 is represented by a horizontal dashed line in the left panels.

Fig. 28 compares the Fourier spectra of the synthetic time series to those of the original time series (as used in Example SigSpecNative, p. 8, and displayed in Fig. 2, p.11. Both signals introduced by the simulator are identified, but 
the prewhitening of the component at 6.25 cycles per day is performed over the whole time base, although the signal is present only in an interval. This introduces additional noise, which causes the signal at 3.99 cycles per day to drop below the significance limit of 5 and avoids the detection of the component at 5.41 cycles per day.

\subsection{Polynomial trend}

The keyword sim:poly is given with five floating-point parameters. They specify

1. the lower time limit,

2. the upper time limit,

3. the coefficient $P_{0}$,

4. the time zeropoint $t_{0}$, and

5. the exponent $X$.

If the exponent is a non-integer number, the simulator evaluates

$$
P(t):=P_{0}\left|t-t_{0}\right|^{X}
$$

instead and produces a power function.

For integer exponents, the trend is generated by the relation

$$
P(t):=P_{0}\left(t-t_{0}\right)^{X} .
$$

Thus a full polynomial may be constructed by multiple keywords sim: poly with different parameters and integer exponents.

If the lower and upper time limits are both set zero, the polynomial trend is generated for the entire time base.

Example. The sample project sim-poly contains the simulation and analysis of 16 individual power functions defined on different time intervals (Fig.29, p. 68). The sampling of the V photometry of IC 4996 \# 89 is used, and the simulator replaces the original observable values, according to the line

sim:replace

in the file sim-poly.ini. The specifications for the power functions are contained in the lines 


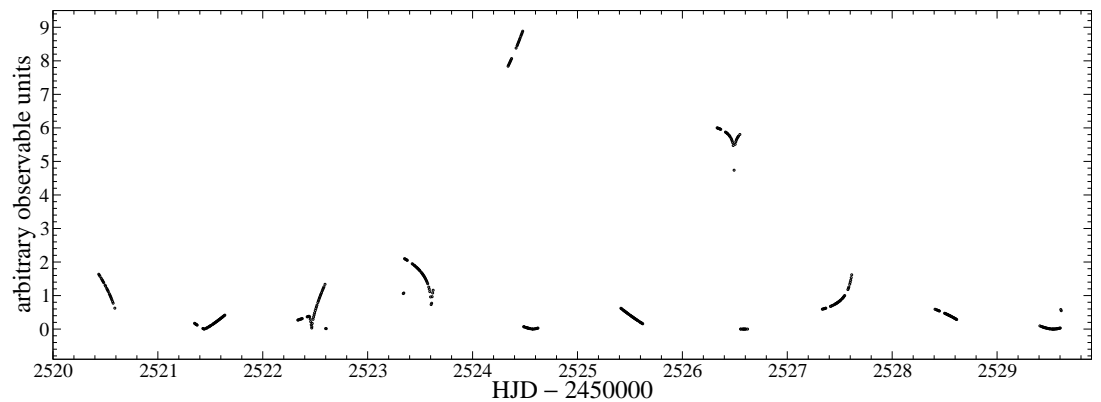

Figure 29: Time series generated by the simulator in the sample project sim-poly. The sampling represents the $\mathrm{V}$ photometry of IC 4996 \# 89 . The simulator replaces the origninal observable by 16 different power functions.

$\begin{array}{lrrrrr}\text { sim:poly } & 2520.215 & 2521.088 & 4.298 & 2520.626 & 0.581 \\ \text { sim:poly } & 2521.088 & 2521.679 & 2.932 & 2521.443 & 1.195 \\ \text { sim:poly } & 2521.679 & 2522.442 & 1.092 & 2522.067 & 1.063 \\ \text { sim:poly } & 2522.442 & 2522.595 & 5.372 & 2522.466 & 0.676 \\ \text { sim:poly } & 2522.595 & 2523.351 & 2.495 & 2522.682 & 2.042 \\ \text { sim:poly } & 2523.351 & 2523.924 & 2.839 & 2523.607 & 0.221 \\ \text { sim:poly } & 2523.924 & 2524.478 & 8.357 & 2525.412 & -0.899 \\ \text { sim:poly } & 2524.478 & 2525.399 & 2.304 & 2524.576 & 1.432 \\ \text { sim:poly } & 2525.399 & 2526.107 & 2.573 & 2525.721 & 1.205 \\ \text { sim:poly } & 2526.107 & 2526.550 & 6.350 & 2526.493 & 0.031 \\ \text { sim:poly } & 2526.550 & 2526.847 & 4.192 & 2526.589 & 2.893 \\ \text { sim:poly } & 2526.847 & 2527.616 & 0.345 & 2527.652 & -0.472 \\ \text { sim:poly } & 2527.616 & 2528.264 & 3.583 & 2527.783 & 0.725 \\ \text { sim:poly } & 2528.264 & 2528.777 & 1.246 & 2528.704 & 0.610 \\ \text { sim:poly } & 2528.777 & 2529.606 & 3.534 & 2529.535 & 1.752 \\ \text { sim:poly } & 2529.606 & 2530.242 & 9.002 & 2529.694 & 1.119\end{array}$

The screen output contains the lines

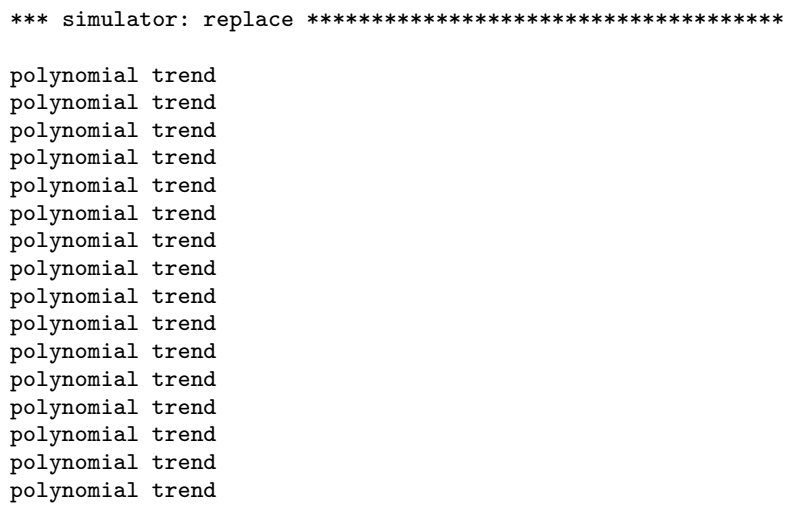


to indicate that the simulator replaces the original observables by the synthetic values, and that 16 power functions are generated.

SIGSPEC detects 19 significant signal components, which are not discussed here.

\subsection{Exponential trend}

The keyword sim: exp is given with five floating-point parameters. They specify

1. the lower time limit,

2. the upper time limit,

3. the coefficient $E_{0}$,

4. the time zeropoint $t_{0}$, and

5. the exponent $X$.

The polynomial trend is generated by the relation

$$
E(t):=E_{0} \mathrm{e}^{X\left(t-t_{0}\right)} .
$$

If the lower and upper time limits are both set zero, the exponential trend is generated for the entire time base.

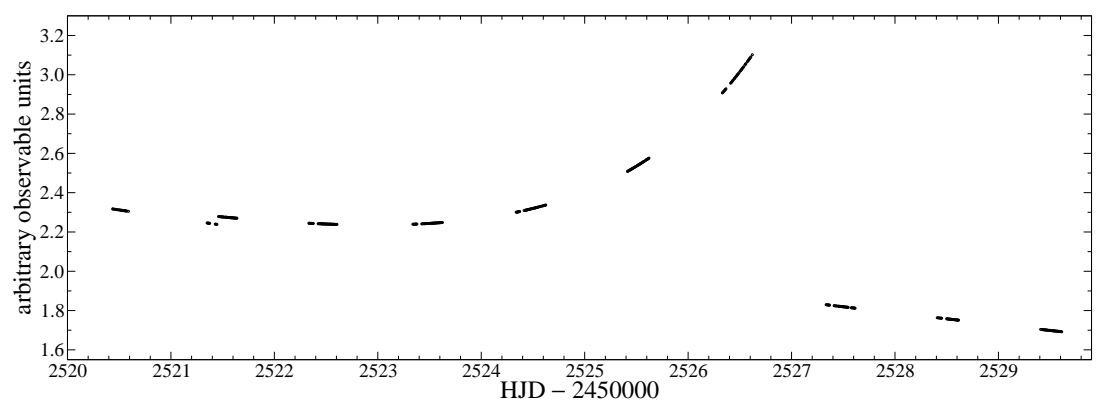

Figure 30: Time series generated by the simulator in the sample project sim-exp. The sampling represents the V photometry of IC 4996 \# 89. The simulator replaces the origninal observable by two exponential functions, one over the entire time base, and the other one on an interval between HJD 2452521.4532 and HJD 2452526.8832.

Example. The sample project sim-exp contains the simulation and analysis of two exponential trends, one over the entire time base, one on a restricted time interval, corresponding to the lines 
sim:exp $2521.45322526 .8832 \quad 1.3256 \quad 2526.7384 \quad 0.65834$

$\begin{array}{llllll}\text { sim:exp } & 0 & 0 & 2.2841 & 2520.8562 & -0.03425\end{array}$

in the file sim-exp.ini. The sampling of the $V$ photometry of IC $4996 \# 89$ is used, and the simulator replaces the original observable values, according to the line

sim:replace

The screen output contains the expression exponential trend to indicate that such a trend is generated. In this example, the entry is found twice. The resulting light curve is displayed in Fig. 30, p. 69.

SigSPEC detects 54 significant signal components, which are not discussed here.

\subsection{Serially correlated noise}

This simulator module produces Gaussian noise the standard deviation of which may vary in time according to a polynomial trend. A serial correlation coefficient between consecutive data points may be specified additionally.

The keyword sim:serial is given with six floating-point parameters. They specify

1. the lower time limit,

2. the upper time limit,

3. the coefficient $\sigma_{0}$ for the standard deviation of the Gaussian noise,

4. the time zeropoint $t_{0}$ for the polynomial trend of the standard deviation,

5. the exponent $X$ for the polynomial trend of the standard deviation, and

6. the serial correlation coefficient.

The standard deviation of the Gaussian noise follows the relation

$$
\sigma(t):=\sigma_{0}\left(t-t_{0}\right)^{X} .
$$

A full polynomial may be constructed by multiple keywords sim:serial with different parameters.

If the lower and upper time limits are both set zero, the noise is generated for the entire time base.

Example. The sample project sim-serial contains the simulation and analysis of serially correlated noise. The sampling of the V photometry of IC 4996 \# 89 is used, and the simulator replaces the original observable values, according to the line 

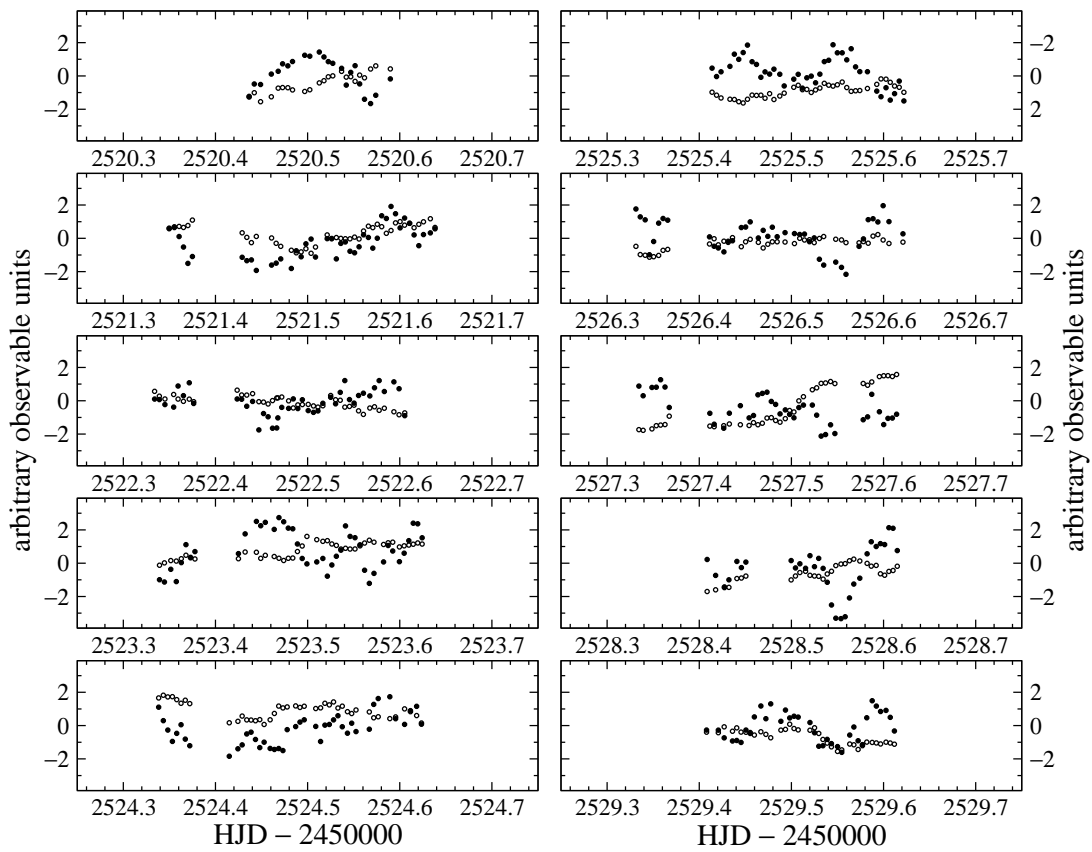

Figure 31: Time series generated by the simulator in the sample projects sim-serial (dots) and sim-temporal (open circles), respectively. The sampling represents the V photometry of IC 4996 \# 89. In both samples, the original observable values are replaced by the simulator.

sim:replace

in the file sim-serial.ini. The line

sim:serial $\begin{array}{lllllll}0 & 0 & 1 & 0 & 0 & 0.8\end{array}$

specifies noise with a constant standard deviation of 1 and a serial correlation coefficient of 0.8 . Setting the first two parameters zero provides synthetic data for the entire time series. The resulting light curve is displayed in Fig. 31. The line

random number generator: file sim-serial.rnd

in the screen output indicates that a file sim-serial.rnd is found and used to initialise the random number generator. If such a file were not present, the system time would be used: 


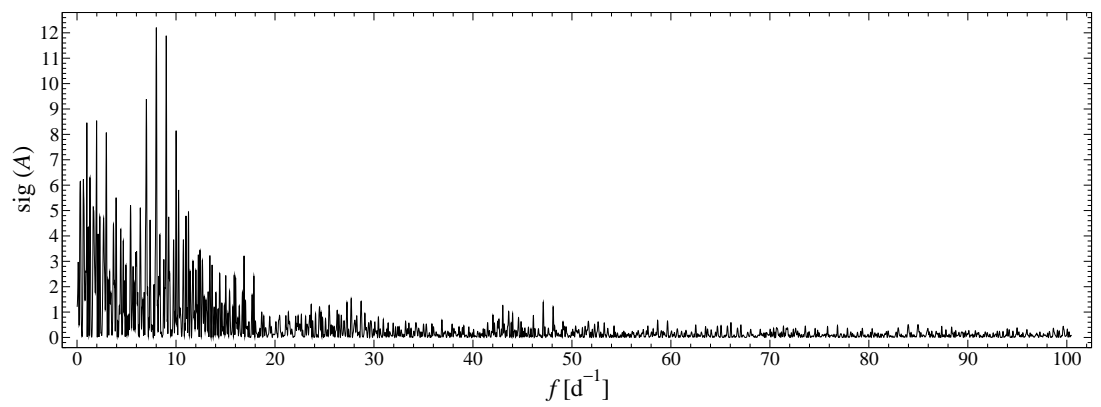

Figure 32: Typical significance spectrum for serially correlated noise, based on the sampling of the V photometry of IC 4996 \# 89. Serial correlation produces systematically higher sigs in the low frequency region.

A significance spectrum is displayed in Fig. 32. The overall shape of the spectrum is typical for serially correlated noise, characterised by higher amplitudes and sigs for low frequencies.

\subsection{Temporally correlated noise}

This simulator module produces Gaussian noise the standard deviation of which may vary in time according to a polynomial trend. A temporal correlation coefficient $R_{T}$ between consecutive data points $t_{n-1}, t_{n}$ may be specified. In contrast to the serial correlation, the temporal correlation takes into account the width of the time interval between pairs of data points, which has implications on the noise behaviour of non-equidistantly sampled data. The serial correlation $R_{S}$ drops exponentially with the distance in time according to

$$
R_{S}:=R_{T}^{t_{n}-t_{n-1}} \text {. }
$$

In this context, the temporal correlation coefficient may be interpreted as the serial correlation coefficient of two data points separated by one unit of time.

The keyword sim:temporal is given with six floating-point parameters. They specify

1. the lower time limit,

2. the upper time limit,

3. the coefficient $\sigma_{0}$ for the standard deviation of the Gaussian noise,

4. the time zeropoint $t_{0}$ for the polynomial trend of the standard deviation, 
5. the exponent $X$ for the polynomial trend of the standard deviation, and

6. the temporal correlation coefficient $R_{T}$.

The standard deviation of the Gaussian noise follows the relation

$$
\sigma(t):=\sigma_{0}\left(t-t_{0}\right)^{X}
$$

A full polynomial may be constructed by multiple keywords sim: temporal with different parameters.

If the lower and upper time limits are both set zero, the noise is generated for the entire time base.

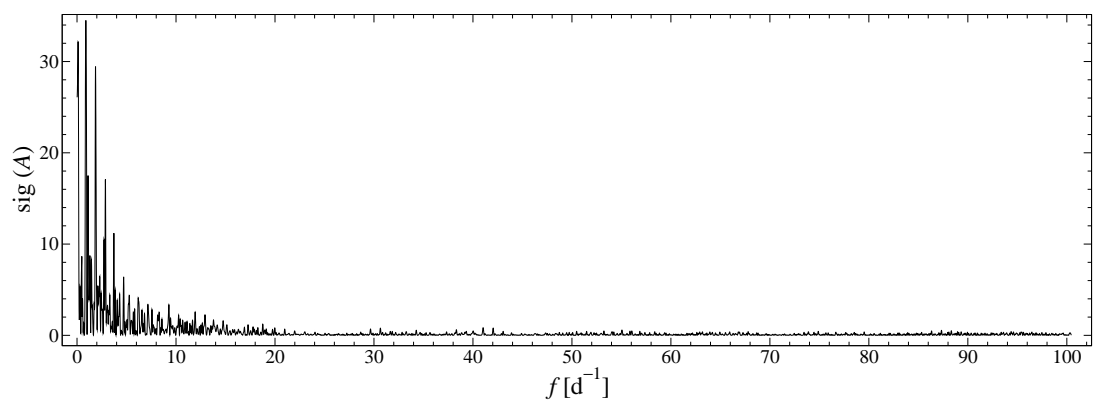

Figure 33: Typical significance spectrum for temporally correlated noise, based on the sampling of the V photometry of IC 4996 \# 89. Temporal correlation produces systematically higher sigs in the low frequency region, which is quite comparable to serial correlation (Fig. 32).

Example. The sample project sim-temporal contains the simulation and analysis of temporally correlated noise. The sampling of the $V$ photometry of IC 4996 \# 89 is used, and the simulator replaces the original observable values, according to the line

sim:replace

in the file sim-temporal.ini. The line

sim:temporal $\quad 0001000.01$

specifies noise with a constant standard deviation of 1 and a temporal correlation coefficient of 0.01 . Setting the first two parameters zero provides synthetic data for the entire time series. The resulting light curve is displayed in Fig. 31. Comparing this light curve to the dataset generated in the project 
sim-serial (p. 70), the correlation between consecutive data points is obviously much stronger in the present example. Using Eq. 25 with a typical sampling interval width of 9 min for the dataset under consideration, the temporal correlation coefficient of 0.01 corresponds to a serial correlation coefficient of $\approx 0.97$.

The significance spectrum displayed in Fig. 33 shows the same overall characteristics as the corresponding spectrum for serially correlated noise (Fig. 32, but the sigs at low frequencies are considerably higher, which is a consequence of the strong serial correlation associated to this setup.

\subsection{Random steps}

This module generates steps following two random processes:

1. the constant attained by the synthetic observable throughout each step follows a Gaussian distribution with an expected value 0 ,

2. a Poisson process is used to define when a step has to be incorporated.

The keyword sim:rndsteps is given with four floating-point parameters. They specify

1. the lower time limit,

2. the upper time limit,

3. the standard deviation of the Gaussian distribution defining the constants attained throughout each step,

4. the expected time range for the Poisson distribution of steps.

If the lower and upper time limits are both set zero, the steps are generated for the entire time base.

Example. The sample project sim-rndsteps illustrates the simulation and analysis of random steps upon the sampling of the $V$ photometry of IC 4996 \# 89. The simulator replaces the original observable values, according to the line

sim:replace

in the file sim-rndsteps.ini. The line

sim:rndsteps $\quad \begin{array}{lllll}0 & 0 & 0.5 & 0.07\end{array}$ 

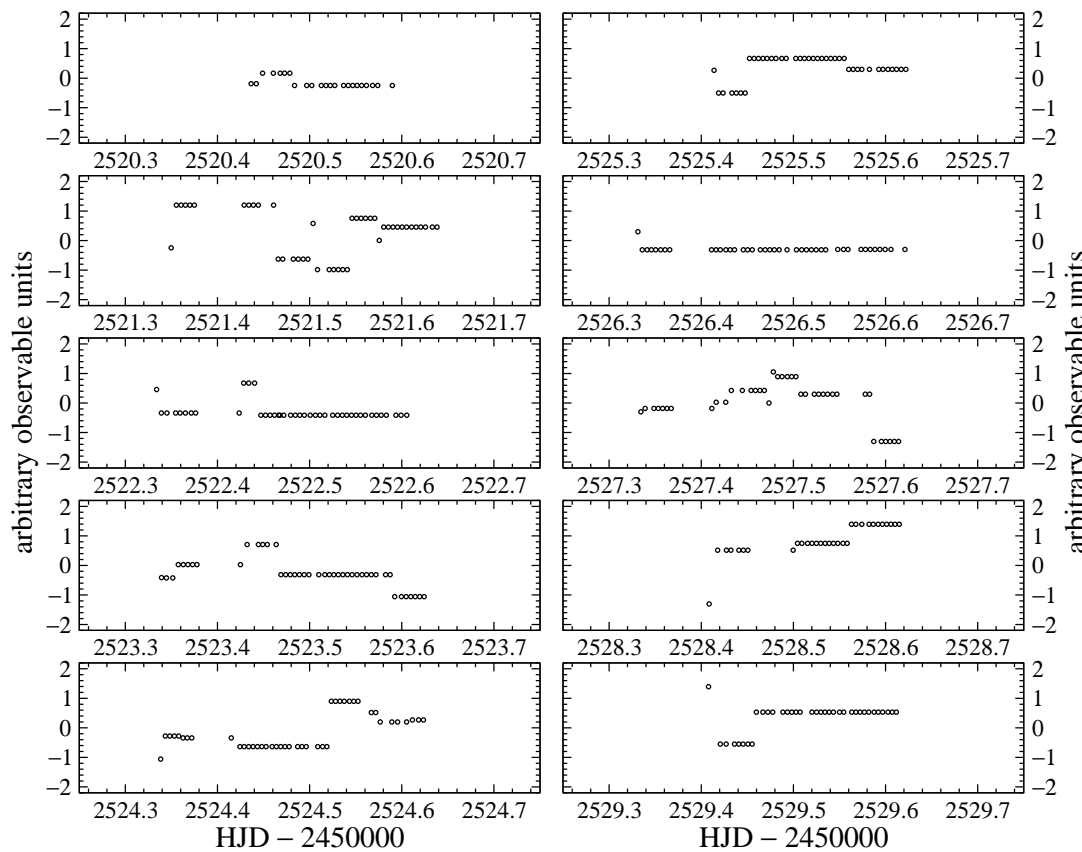

Figure 34: Time series generated by the simulator in the sample project sim-rndsteps. The sampling represents the V photometry of IC 4996 \# 89 . The original observable values are replaced by the simulator.

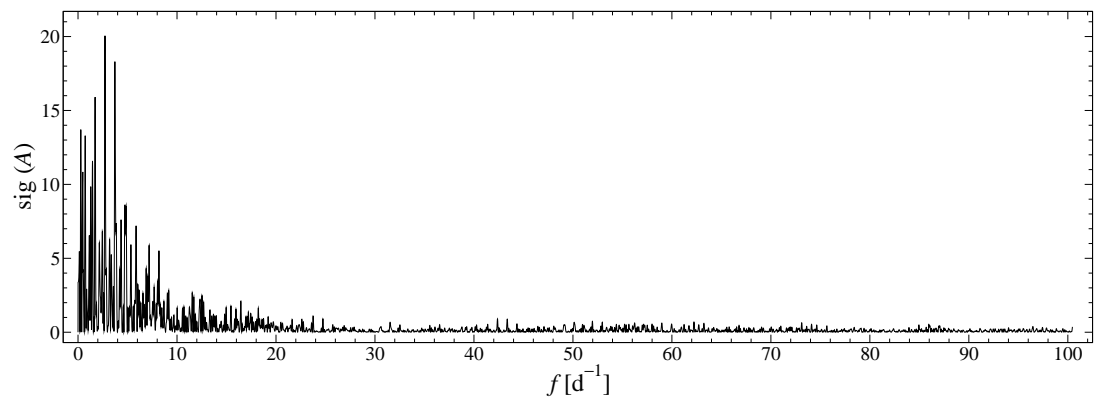

Figure 35: Typical significance spectrum for random steps, based on the sampling of the V photometry of IC 4996 \# 89. Each constant in the step function displayed in Fig. 34 contributes a spectral window to this DFT.

in the file sim-rndsteps. ini produces random steps the values of which are distributed according to a Gaussian with standard deviation 0.5. The expected 
distance in time of consecutive steps 0.07 days. The resulting light curve is displayed in Fig. 34.

Since the observables are constant between the steps, one may consider each of the corresponding time intervals to contribute a spectral window to the DFT, or significance spectrum, correspondingly. The significance spectrum associated to the light curve in Fig. 34 is displayed in Fig. 35 and represents such a superposition of spectral windows.

\subsection{Zero-mean adjustment}

The keyword sim:zeromean may be used to adjust the mean value of the time series (or a subset) to zero. It is given with two floating-point parameters,

1. the lower time limit, and

2. the upper time limit.

If the lower and upper time limits are both set zero, the mean value of the entire synthetic time series is adjusted to zero. This option was adopted for consistency, but does not provide additional functionality, because a zero-mean correction of the whole data set is performed at every step of the prewhitening cascade by default.

Example. In the sample project sim-zeromean, SIGSPEC models the same time series as in the project sim-poly (p.67), according to the first part of the file sim-zeromean.ini:

$\begin{array}{lrrrrr}\text { sim:poly } & 2520.215 & 2521.088 & 4.298 & 2520.626 & 0.581 \\ \text { sim:poly } & 2521.088 & 2521.679 & 2.932 & 2521.443 & 1.195 \\ \text { sim:poly } & 2521.679 & 2522.442 & 1.092 & 2522.067 & 1.063 \\ \text { sim:poly } & 2522.442 & 2522.595 & 5.372 & 2522.466 & 0.676 \\ \text { sim:poly } & 2522.595 & 2523.351 & 2.495 & 2522.682 & 2.042 \\ \text { sim:poly } & 2523.351 & 2523.924 & 2.839 & 2523.607 & 0.221 \\ \text { sim:poly } & 2523.924 & 2524.478 & 8.357 & 2525.412 & -0.899 \\ \text { sim:poly } & 2524.478 & 2525.399 & 2.304 & 2524.576 & 1.432 \\ \text { sim:poly } & 2525.399 & 2526.107 & 2.573 & 2525.721 & 1.205 \\ \text { sim:poly } & 2526.107 & 2526.550 & 6.350 & 2526.493 & 0.031 \\ \text { sim:poly } & 2526.550 & 2526.847 & 4.192 & 2526.589 & 2.893 \\ \text { sim:poly } & 2526.847 & 2527.616 & 0.345 & 2527.652 & -0.472 \\ \text { sim:poly } & 2527.616 & 2528.264 & 3.583 & 2527.783 & 0.725 \\ \text { sim:poly } & 2528.264 & 2528.777 & 1.246 & 2528.704 & 0.610 \\ \text { sim:poly } & 2528.777 & 2529.606 & 3.534 & 2529.535 & 1.752 \\ \text { sim:poly } & 2529.606 & 2530.242 & 9.002 & 2529.694 & 1.119\end{array}$

This block of sim:poly keywords is followed by a corresponding block of sim:zeromean keywords:

sim:zeromean sim:zeromean sim:zeromean
2520.2152521 .088

2521.0882521 .679

2521.6792522 .442 


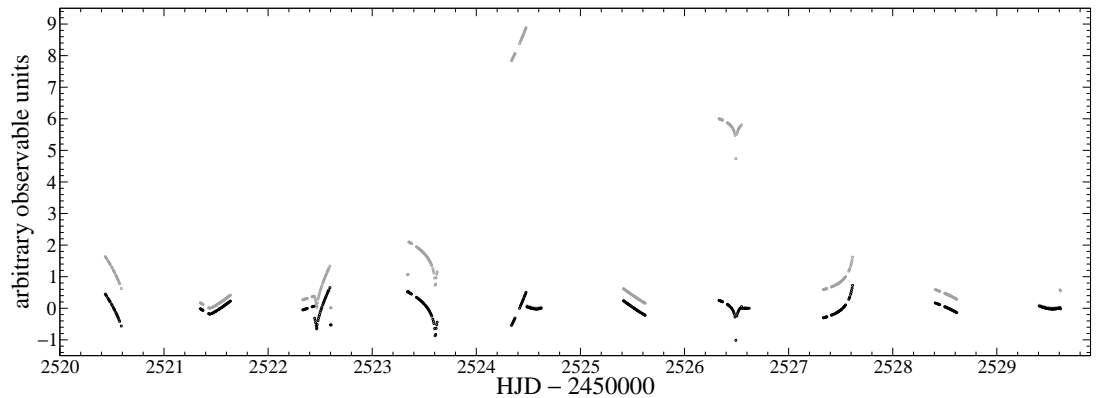

Figure 36: Time series generated by the simulator in the sample project sim-zeromean. The sampling represents the V photometry of IC 4996 \# 89 . First the simulator generates a set of power functions over intervals within the time series (grey), then the actual light curve (black) is produced by shifting the mean observable for each power function to zero individually.

$\begin{array}{lll}\text { sim:zeromean } & 2522.442 & 2522.595 \\ \text { sim:zeromean } & 2522.595 & 2523.351 \\ \text { sim:zeromean } & 2523.351 & 2523.924 \\ \text { sim:zeromean } & 2523.924 & 2524.478 \\ \text { sim:zeromean } & 2524.478 & 2525.399 \\ \text { sim:zeromean } & 2525.399 & 2526.107 \\ \text { sim:zeromean } & 2526.107 & 2526.550 \\ \text { sim:zeromean } & 2526.550 & 2526.847 \\ \text { sim:zeromean } & 2526.847 & 2527.616 \\ \text { sim:zeromean } & 2527.616 & 2528.264 \\ \text { sim:zeromean } & 2528.264 & 2528.777 \\ \text { sim:zeromean } & 2528.777 & 2529.606 \\ \text { sim:zeromean } & 2529.606 & 2530.242\end{array}$

This block is responsible for shifting the mean observable to zero for each synthesized power function.

Fig. 36 compares the corresponding light curve with the light curve generated in the project sim-poly (see also Fig. 29). The 16 significant signal components detected by SIGSPEC are of minor interest and not discussed here.

\section{Signal-to-Noise Ratio and Lomb-Scargle Periodogram}

As pointed out by Reegen (2007), the SIGSPEC method represents a tool for an iterative frequency analysis of a zero-mean corrected time series superior to signal-to-noise ratio estimation (Breger et al. 1993) and Lomb-Scargle periodogram (Lomb 1976; Scargle 1982). However, in some situations these alternative methods may be desired or even more reasonable. Namely the LombScargle periodogram represents the optimum statistical approach to the problem if the mean observable is meaningful rather than set zero arbitrarily. The 
relations between sig and signal-to-noise ratio or Lomb-Scargle periodogram, respectively, are introduced and discussed by Reegen (2007).

In order to meet a user's requirement of signal-to-noise ratio-based DFT analysis or Lomb-Scargle periodograms as well, the SIGSPEC software offers the option to perform an analysis relying on amplitude signal-to-noise ratios by providing the keyword DFT in the .ini file. If this keyword is specified, all SIGSPEC computations rely on the approximation of sig by the amplitude signal-to-noise ratio according to

$$
\operatorname{sig}(A) \approx \frac{K \log \mathrm{e}}{4} \frac{A^{2}}{\left\langle x^{2}\right\rangle},
$$

where $K$ represents the number of time series data, $A$ denotes the Fourier amplitude, and $\left\langle x^{2}\right\rangle$ refers to the variance of the observable.

Second, the keyword Lomb forces SIGSPEC to evaluate Lomb-Scargle periodograms rather than significance spectra. In this case, the sig is approximated by

$$
\operatorname{sig}(A) \approx \frac{K \log \mathrm{e}}{4} \frac{P_{\mathrm{LS}}}{\left\langle x^{2}\right\rangle},
$$

where $P_{\mathrm{LS}}$ denotes the power level in terms of the Lomb-Scargle periodogram.

Example. In the sample projects DFT and $\mathrm{L}-\mathrm{S}$, the input time series represents the $V$ photometry of IC 4996 \# 89.

The file DFT . ini contains a single entry

DFT

which forces SIGSPEC to rely on the signal-to-noise ratio of DFT amplitudes. The screen output is:

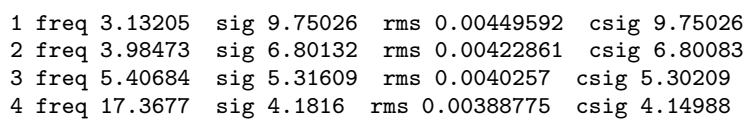

The file L-S. ini contains a single keyword

Lomb

and SigSpec uses the Lomb-Scargle periodogram rather than sig for all computations. The screen output is: 


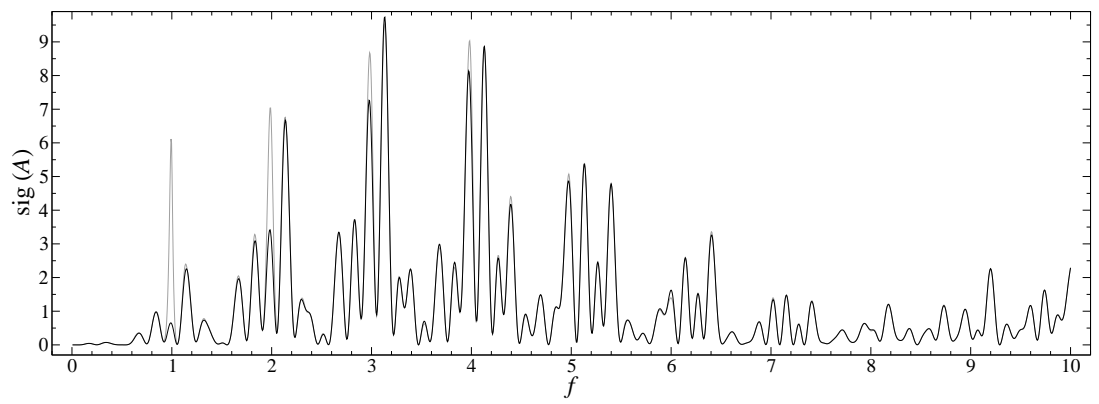

Figure 37: Significance spectrum of the V photometry of IC 4996 \# 89 (grey) and approximation by the signal-to-noise ratio of DFT amplitudes (black).

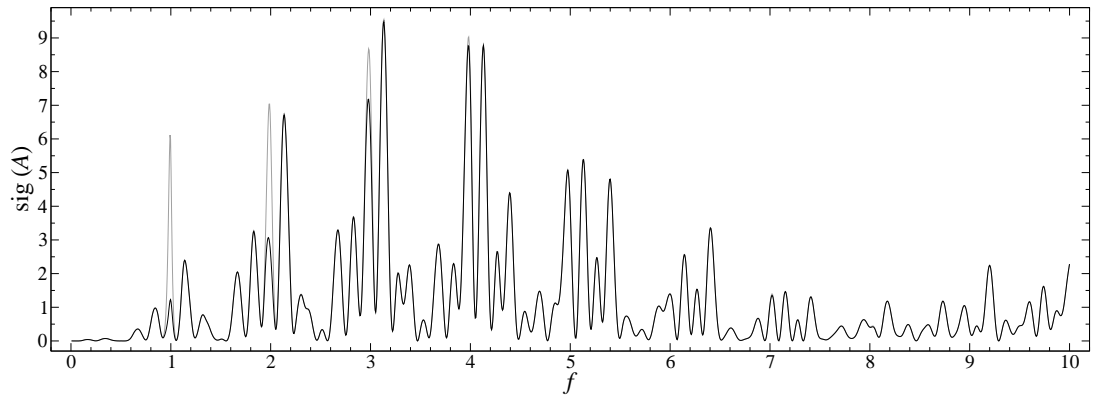

Figure 38: Significance spectrum of the V photometry of IC 4996 \# 89 (grey) and approximation by the Lomb-Scargle periodogram (black).

The significance spectrum of the input time series is compared to the approximations by DFT amplitude signal-to-noise ratio and Lomb-Scargle periodogram in Figs. 37 and 38, respectively.

A comparison of the two outputs and the screen output of the corresponding sig-based application (Example SigSpecNative, p. 9) reveals slightly different signal components. Especially for the second component the frequency of which is close to an integer multiple of 1 cycle per day and therefore susceptible to alias, the results are different for all three methods. However, the frequencies, amplitudes and phases in the files result. dat are in good agreement and reflect the numerical uncertainties of the MultiSine fitting procedure only. 


\section{Frequently Asked Questions}

This section contains questions frequently asked by users familiar to common methods of astronomical time series analysis involving signal-to-noise ratio estimation in power spectra and consecutive prewhitenings. The intention is to clarify the differences between these classical techniques and SIGSPEC from the user's perspective.

\subsection{Changing sig in a prewhitening sequence}

Given a time series showing two different peaks in the power spectrum, prewhitening of the dominant signal usually does not cause a major change in the height of the secondary peak in the spectrum of the residuals. Why does the corresponding sig change?

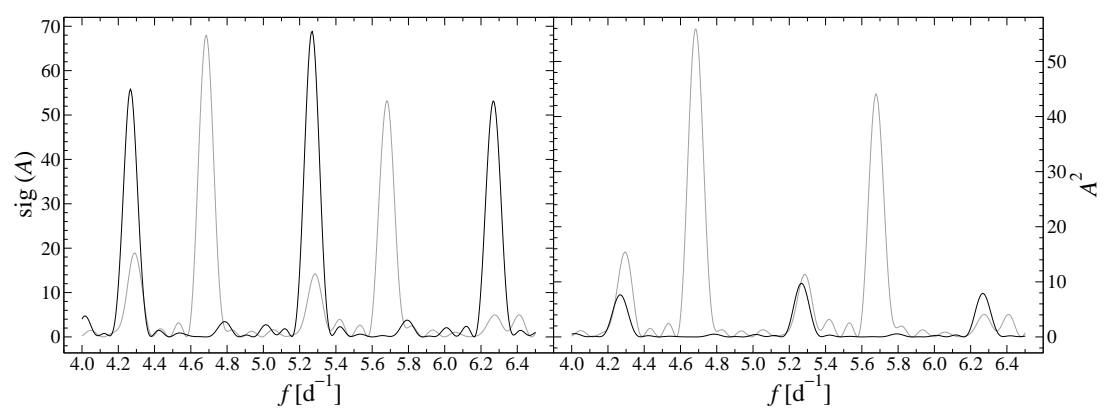

Figure 39: Grey graphs: sig (left) and power (squared amplitude) spectra (right) of a synthetic time series containing two signals plus noise. The sampling represents the $\checkmark$ photometry of IC 4996 \# 89. Black graphs: spectra after subtracting the dominant signal $\left(f=4.68573 d^{-1}\right)$.

The situation is illustrated in Fig. 39 displaying the sig (left panel) and power (right panel) spectra (in this sample just squared amplitude) generated by a synthetic time series. It consists of two sinusoidal signals, $f_{1}=4.68573 \mathrm{~d}^{-1}$, $A_{1}=7.27$, and $f_{2}=5.26934 \mathrm{~d}^{-1}, A_{2}=3.31$, plus noise with unit rms error. The plots contain a comparison of the initial spectra (grey) and the spectra after subtraction of the first signal component. In the right panel, the power associated to the peak at $5.27 \mathrm{~d}^{-1}$ differs only slightly between the two iterations, whereas the corresponding sig in the left panel increases dramatically in the second iteration.

The reason for this behaviour is that the sig refers to the probability of a random time series with the same rms error as the given one to produce a peak like the given one. In the first iteration, the sig calculation is based on the 
initial time series (rms error 5.84), and in the second iteration, it relies on the residual time series after prewhitening of the peak at $f_{1}$, the rms error of which is 2.46. The ratio of rms errors $(\approx 2.4)$ is in agreement with the root ratio of sigs at $f_{2}$ in the two iterations $(\approx 2.2)$.

This effect is more prominent for high sigs, because in this case prewhitening causes a major change in the statistical properties of the time series. If a peak with a low sig is prewhitened, the time series is affected marginally, and correspondingly, the sigs of other signals do not change much.

\subsection{The effect of binning}

Consider a time series representing a signal plus noise. If the data points are grouped into bins, the noise of the binned observables will reduce by the square root of the number of points in each bin. On the other hand, the number of data points the time series consists of reduces by the same amount. Since these two effects cancel each other, the noise level in the power spectrum will be the same for unbinned and binned data. What is the corresponding situation in terms of significance?

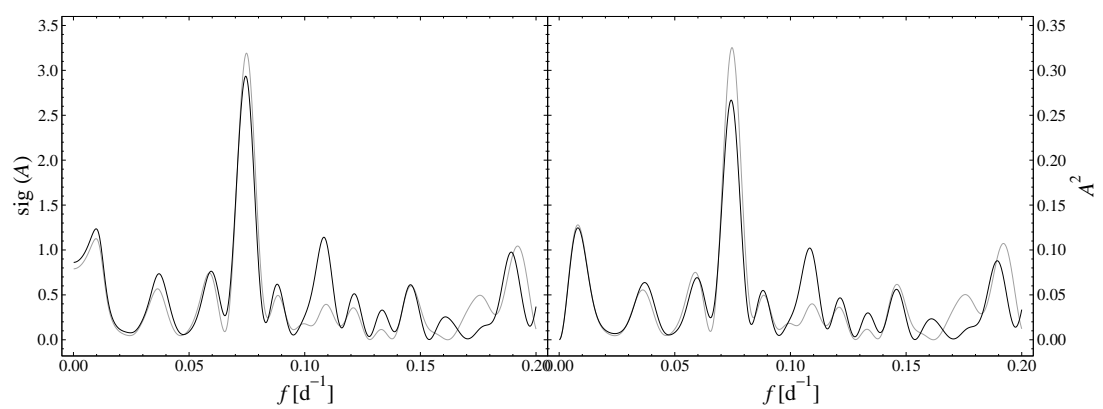

Figure 40: Grey graphs: sig (left) and power (squared amplitude) spectra (right) of a synthetic time series (100 equidistant data points) containing a sinusoidal signal plus noise with a standard deviation of 1 . The signal amplitude is 0.5 . Black graphs: same for time series data grouped into bins of two points. The resulting time series consists of 50 data points.

Fig. 40 contains the significance (left) and power (squared amplitude) spectra (right) of a synthetic time series containing a sinusoidal signal with a frequency of $0.075832 \mathrm{~d}^{-1}$ plus Gaussian noise with a standard deviation of 1 . The signal amplitude is 0.5 , providing an amplitude signal-to-noise ratio of 5.64 . All corresponding plots are displayed in grey colour. The black graphs represent the spectra generated by a binned version of the time series: each bin contains two data points, and the observable is the arithmetic mean. 
In terms of sig as well as amplitude, binning affects neither the peak nor the mean amplitude remarkably: the reduced number of data points would increase the amplitude noise, but this effect is mitigated by the fact that binning reduces the rms residual in the time domain. For a multi-sine signal plus white noise, the number of significant peaks in a given frequency range will hardly be modified by data binning. A considerable change of these sigs by binning is an indication of the noise not being white. A correlation between consecutive measurements in the time series would be a reasonable explanation for such a behaviour.

\subsection{Binning of extremely strong signals}

If an extremely strong signal is binned, the sig changes, whereas the signal amplitude and the noise level do not. Why?

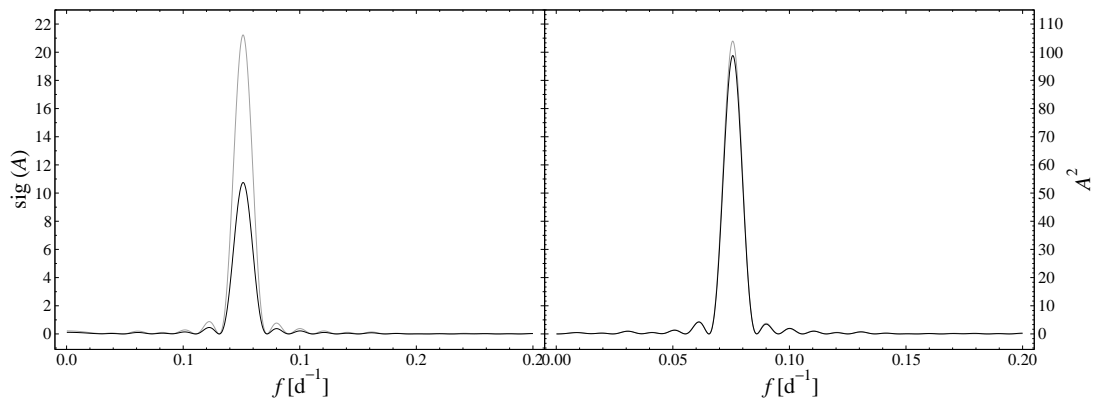

Figure 41: Grey graphs: sig (left) and power (squared amplitude) spectra (right) of a synthetic time series (100 equidistant data points) containing a sinusoidal signal plus noise with a standard deviation of 1 . The signal amplitude is 10 . Black graphs: same for time series data grouped into bins of two points. The resulting time series consists of 50 data points.

Fig. 41 contains the significance (left) and power (squared amplitude) spectra (right) of a synthetic time series containing a sinusoidal signal with a frequency of $0.075832 \mathrm{~d}^{-1}$ plus Gaussian noise with a standard deviation of 1 . The signal amplitude of 10 is associated to an ampltiude signal-to-noise ratio of more than 100. All corresponding plots are displayed in grey colour. The black graphs represent the spectra generated by a binned version of the time series: each bin contains two data points, and the observable is the arithmetic mean.

For both strong and weak signals, binning affects neither the peak nor the mean amplitude remarkably: the reduced number of data points would increase the amplitude noise, but this effect is mitigated by the fact that binning reduces the rms residual in the time domain. 
In terms of sig the situation is different: for very strong signals, the peak sig is reduced by binning. Classical techniques prewhiten a peak under consideration and employ the residuals to estimate a noise level. SIGSPEC does not imply any prewhitening. In the case of a dominant signal plus a tiny scatter, the unbinned and binned data have comparable rms deviations, which are mainly determined by the signal. In the frequency domain, only the reduced number of binned data points comes into play.

Very strong signals let the sig drop to $\approx \frac{1}{N}$ by forming groups of $N$ data points: in Fig. 41, left panel, the grey peak is about twice as high as the black peak.

\subsection{Linear interpolation: more information?}

Consider a time series representing a signal plus noise. Generating additional data points through linear interpolation increases the sig of the signal peak, although the power spectrum remains practically unchanged. This provides the possibility to boost signal sigs artificially, although the amount of information contained by the time series does not increase. Does this make sense?

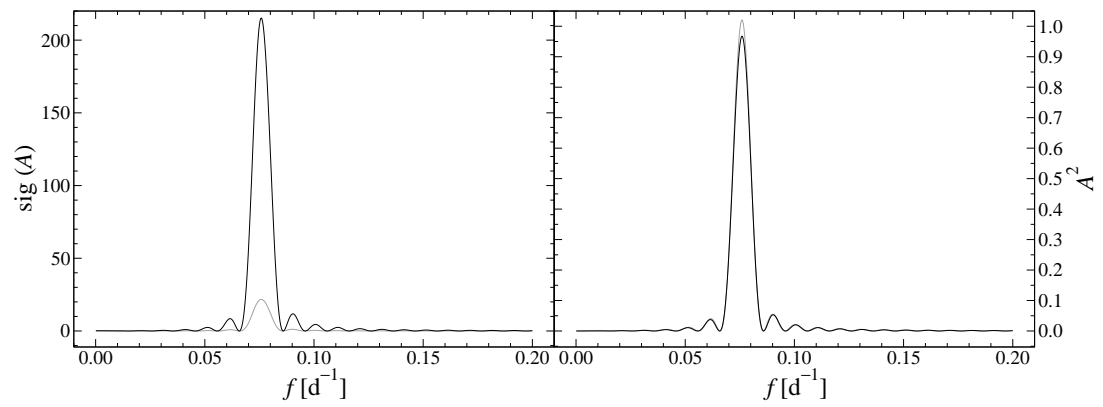

Figure 42: Grey graphs: sig (left) and power (squared amplitude, logarithmic scale) spectrum (right) of a synthetic time series (100 equidistant data points) containing a sinusoidal signal without noise. Black graphs: same for a new time series generated by inserting 9 additional linearly interpolated points such that the result is an equidistantly sampled dataset consisting of 991 points.

Fig. 42 displays the sig (left) and power (squared amplitude, right) spectrum of an equidistantly sampled time series consisting of 100 data points and representing a sinusoidal signal with a frequency of $0.075832 \mathrm{~d}^{-1}$ and an amplitude of 1 in black colour. No noise is added. Based on this time series, a new dataset is generated: between each pair of data points, 9 additional, equidistant data points are inserted. The observables are assigned by linear interpolation. The 
number of data points in this new time series is thus 991 . The corresponding spectra are shown in grey colour. The longer dataset generates a peak significance that is roughly ten times higher than the initial one, whereas the power spectrum remains practically unchanged. Only the fact that the linear interpolation does not reveal the "true" observables that would be generated by the signal exactly is responsible for a small deviation of the black graph from the grey one.

The explanation for this behaviour is quite similar to the previous section "The effect of binning", p. 81, and correspondingly, the effect is mitigated for very noisy signals. Therefore in practical applications, it will be impossible to enhance the capability of a frequency analysis by artificially introducing new data points.

\subsection{Which sig threshold is reasonable?}

Occasionally, sigs or sig limits are shifted by $\log \frac{K}{2}, K$ denoting the number of time series data points. Which sig threshold is the true one?

In fact both versions are correct, but they apply to different questions. The version without $\log \frac{K}{2}$ refers to the probability that an amplitude level (a peak) at a given frequency and phase occurs by chance. The version including $\log \frac{K}{2}$ corresponds to the probability that the highest out of $\frac{K}{2}$ independent peaks occurs by chance. ${ }^{*}$ According to the sampling theorem, the DFT of $K$ data points (a system with $K$ degrees of freedom) produces $\approx \frac{K}{2}$ independent frequencies in Fourier space, if the sampling is equidistant. Although there is no explicit prescription where to find a set of independent frequencies for nonequidistant sampling, the system will still have $K$ degrees of freedom, and the statistical considerations will be essentially the same.

A simple experiment makes the situation clearer: we roll a dice and obtain the result "4". The probability that such an experiment returns at least "4" (i. e. " 4 ", " 5 " or " 6 ") is, of course, $50 \%$. This refers to the examination of an individual peak without respect to all the others in the spectrum. If we roll 10 dices, the probability for at least one showing " 4 " or more is dramatically higher, namely $>99.9 \%$. This refers to examining the highest out of 10 peaks. The increasing probability of obtaining such a result by chance corresponds to a decreasing significance of the result.

* Note by M. Gruberbauer: The standard SIGSPEC output corresponds to "the version without $\log \frac{K}{2}$ ". See Section 5.5 .2 of Reegen (2007) for a more detailed explanation of how the significance can be shifted by $\log \frac{K}{2}$ in order to be comparable to the false-alarm probability presented in Scargle (1982). 


\section{Keywords Reference}

This section is a compilation of all keywords accepted by SigSPEC. A brief description of arguments and default values is given. The type of argument is provided by either <int $\rangle$ or $\langle$ double $\rangle$, and default values are given in parentheses, e. g. (2). Empty parentheses indicate that there is no default setting.

antialc:adopt <int> (1)

number of AntiAIC test iterations adopted for the main prewhitening cascade, p. 48

antialc: depth <int> (automatic)

AntiAIC computation depth, p. 48

parameter: number of iterations used for peak combination testing

default: $\frac{1}{\sqrt{p_{\mathrm{al}}}}$, where $p_{\mathrm{al}}$ is the AntiAIC parameter, rounded to the successive integer value

antialc:par <double> ()

AntiAIC parameter $p_{\mathrm{al}}$ : sig limit relative to maximum for the selection of candidate peaks $(0 \ldots$ use the sig limit siglimit instead $)$, p. 47

antialc:siglimit <double> ()

significance limit for the AntiAIC candidate peak selection (no significance limit by default; the limit defined by the keyword siglimit is used instead), p. 47

col:obs <int> (2)

observable column index (unique), starting with 1, p. 13

col:ssid <int> ()

subset identifier column index (also multiple), starting with 1, p. 15

col:time <int> (1)

time column index (unique), starting with 1, p. 13

col:weights <int> ()

weights column index (also multiple), starting with 1, p. 13 
comp <int> ()

specifies the file indicated by the parameter as comparison dataset, p. 59

correlograms <int> <int> <int> () ()

specifications for correlogram files c\#iteration\#. dat, p. 42

parameters:

- correlogram order (maximum index lag), default: half of the number of time series data points,

- number of files to generate $(<0$ for all correlogram files, default: no correlogram computation),

- step width (number of iterations) for output.

csiglimit <double> ()

lower cumulative sig limit, p. 23

deftype <target/comp/skip> (target)

specifies the type of dataset to be assigned to a time series by default, p. 59

DFT

forces SIGSPEC to approximate all sigs by signal-to-noise ratios of DFT amplitudes, p. 78

diff : comp

specifies the DFT amplitude spectrum of the comparison datasets to be calculated through a weighted mean of Fourier vectors, p. 60

diff : compalign

specifies the DFT amplitude spectrum of the comparison datasets to be calculated as a weighted mean of Fourier amplitudes, p. 60

This setting forces SigSPEC to take into account also correlated signal components that lag in phase with respect to each other and the target dataset, respectively.

$\operatorname{diff}:$ off

switches off the differential significance computation (default), p. 60 
freqspacing <double> ()

spacing between consecutive frequencies [inverse time units], p. 20

harmonics <int> ()

activates the simultaneous analysis of a fundamental plus harmonics (the frequencies of which are integer multiples of the fundamental) the number of which is specified by the parameter, p. 51

iterations <int> ()

number of prewhitening iterations, p. 23

lfreq <double> (0)

lower frequency limit [inverse time units], p. 18

Lomb

forces SIGSPEC to approximate all sigs by the Lomb-Scargle periodogram, p. 78

mfstart <int> (0)

index of the first time series input file to apply the MultiFile mode to, p. 57

mstracks <int> <int> ()

MultiSine tracks are written to files m\#index\#. dat, where \#index\# refers to the signal component in the result files. The parameters are

1. the maximum number of iterations for which to write entries to the MultiSine track files, and

2. the step width (number of iterations) for output, p. 37.

The file name may be assigned additional indices for Time-Resolved analysis (p. 42) and/or MultiFile mode (p. 56).

multifile <int> ()

activates MultiFile mode, p. 56

parameter: maximum index of time series input files $(\leq 0 \ldots$ infinite $)$ 
multisine:lock

forces SIGSPEC to use the "raw" frequencies, amplitudes, and phases (without MultiSine fitting) for the subsequent analysis, p. 23.

multisine:newton <double> <double> <double> ( $\left.\begin{array}{lllll}0.000001 & 1 & 0.000001\end{array}\right)$

accuracy parameters for the MultiSine least-squares fits

1. scaling factor for the overall precision of resulting frequencies,

2. degree of dependence of the frequency accuracy on the peak sig,

3. the minimum relative improvement of rms residual between consecutive iterations to continue the fitting process, p. 22.

multisine: unlock

forces SigSpec to use the frequencies, amplitudes, and phases improved by MultiSine least-squared fits for the subsequent analysis (default), p. 23.

nycoef <double> $(0.5)$

Nyquist Coefficient (between 0 and 1), p. 19

nyscan

Nyquist Coefficients for the specified frequency range (file nycoef dat or <\#multifile\#>.nycoef.dat), p. 20

osratio <double> (20)

oversampling ratio, p. 21

phdist: cart

generates a Phase Distribution Diagram in three-dimensional cartesian coordinates, p. 35

phdist: colmodel:lin

specifies the linear colour model, i.e., phase probability density is used as a colour scale, p. 35 
phdist: colmodel :rank

specifies the rank colour model, i. e., the rank in an ascending sequence of sock significances is used as a colour scale, p. 35

phdist:colour <double $>$ double $\rangle\langle$ double $\rangle\langle$ double $\rangle$

A set of phdist:colour lines defines an RGB path for colourising the Phase Distribution Diagram, p. 35.

parameters:

- red channel (0...255)

- green channel (0...255)

- blue channel (0...255)

- scale

The scale parameter refers directly to probability density of phases in case of phdist:colmodel:lin, or to a fractile of probability density on the interval $[0,1]$ in case of phdist: colmodel:rank.

phdist: cyl

generates a Phase Distribution Diagram in three-dimensional cylindrical coordinates (default setting), p. 35. The frequency is the height axis, the phase is the azimuth angle, and the radial coordinate refers to the probability density of phase.

phdist:fill <double> (0)

specifies a filling factor to compute extra frequencies if the difference of phase PDFs between two adjacent frequencies is too high, p. 35.

parameter: number of additional frequencies per unit probability density (difference between two adjacent frequencies)

phdist:phases <int> ()

generates a Phase Distribution Diagram for the sampling of the given time series, p. 34. By default, no Phase Distribution Diagram is computed.

parameter: number of phase angles in the interval $[0, \pi[$, if the maximum probability density is $\leq 1$. Between 1 and 2 , twice this number is used, and so on. This enhances the visibility of the Phase Distribution Diagram also in frequency and phase regions associated with a very eccentric phase distribution. 
preview <double> ()

generates a preview, p. 40. Instead of a prewhitening cascade, only one significance spectrum is computed. All local maxima above the specified significance limit are written to a file preview.dat. By default, no preview is computed.

parameter: significance limit

profile

SIGSPEC generates a file profile. dat containing the sampling profile for the given time series, p. 30. By default, the file profile. dat is not generated.

This keyword is ignored in MultiFile mode, where sampling profiles are calculated and written to files whenever required by the program. See "MultiFile Mode", p. 56 for further information.

residuals <int> <int> ()

output files containing residual time series (only residuals . dat for the residuals after prewhitening all significant compontents by default). The parameters are

1. the maximum number of iterations (files t\#iteration\#.dat), and

2. the step width (number of iterations) for output, p. 27.

The file name may be assigned additional indices for Time-Resolved analysis (p. 42) and/or MultiFile mode (p. 56).

results <int> <int> ()

output files containing a list of significant signal components. The default setting is to produce only a file result. dat for the final list. The parameters are

1. the maximum number of iterations for which to write additional result files r\#iteration\#.dat, and

2. the step width (number of iterations) for output, p. 28.

The file name may be assigned additional indices for Time-Resolved analysis (p. 42) and/or MultiFile mode (p. 56).

siglimit <double> (5)

lower sig limit (0 to deactivate), p. 23 
sim : add

add synthetic data to given observable, p. 63

sim: exp <double $>\langle$ double $\rangle\langle$ double $\rangle\langle$ double $\rangle\langle$ double $\rangle$ ()

exponential trend, p. 69

parameters:

- lower time limit [time units]

- upper time limit [time units]

- scale

- time zeropoint [time units]

- exponent

sim:off

deactivate simulator (default), p. 63

sim:poly $\langle$ double $\rangle\langle$ double $\rangle\langle$ double $\rangle\langle$ double $\rangle\langle$ double $\rangle$ ()

polynomial trend, p. 67

parameters:

- lower time limit [time units]

- upper time limit [time units]

- scale

- time zeropoint [time units]

- exponent

full polynomial by multiple declaration with different scales, time zeropoints, and exponents

sim:replace

replace given observable by synthetic data, p. 63 
sim:rndsteps <double> <double> <double> <double> ()

random steps, p. 74

parameters:

- lower time limit [time units]

- upper time limit [time units]

- standard deviation for Gaussian distribution of (constant) step values

- expected time range for Poisson distribution of steps [time units]

sim: serial <double $\rangle\langle$ double $\rangle\langle$ double $\rangle\langle$ double $\rangle\langle$ double $\rangle\langle$ double $\rangle()$ serially correlated noise, p. 70

parameters:

- lower time limit [time units]

- upper time limit [time units]

- scale for standard deviation

- time zeropoint for polynomial trend of standard deviation [time units]

- exponent for polynomial trend of standard deviation

- serial correlation coefficient

full polynomial by multiple declaration with different scales, time zeropoints, and exponents

sim:signal <double $>\langle$ double $\rangle\langle$ double $\rangle\langle$ double $\rangle\langle$ double $\rangle$ ()

sinusoidal signal, p. 64

parameters:

- lower time limit [time units]

- upper time limit [time units]

- amplitude

- time zeropoint [time units]

- frequency [inverse time units] 
sim:temporal <double $\rangle\langle$ double $\rangle\langle$ double $\rangle\langle$ double $\rangle\langle$ double $\rangle\langle$ double $\rangle$ () temporally correlated noise, p. 72 parameters:

- lower time limit [time units]

- upper time limit [time units]

- scale for standard deviation

- time zeropoint for polynomial trend of standard deviation [time units]

- exponent for polynomial trend of standard deviation

- temporal correlation coefficient

full polynomial by multiple declaration with different scales, time zeropoints, and exponents

sim:zeromean <double> <double> ()

zero-mean adjustment, p. 76

parameters:

- lower time limit [time units]

- upper time limit [time units]

skip <int> ()

forces SIGSPEC to skip the file indicated by the parameter, p. 59

sock: cart

generates a Sock Diagram in three-dimensional cartesian coordinates, p. 32

sock: colmodel: lin

specifies the linear colour model, i. e., sock significance is used as a colour scale, p. 32

sock: colmodel:rank

specifies the rank colour model, i. e., the rank in an ascending sequence of sock significances is used as a colour scale, p. 32 
sock: colour <double $\rangle\langle$ double $\rangle\langle$ double $\rangle\langle$ double $\rangle$

A set of sock:colour lines defines an RGB path for colourising the Sock Diagram, p. 33.

parameters:

- red channel (0..255)

- green channel $(0 \ldots 255)$

- blue channel (0...255)

- scale

For the linear colour model selected by the keyword sock:colmodel:lin, the scale parameter refers directly to sock significance. If the rank colour model is selected (sock: colmodel:rank), it refers to a fractile of sock significance on the interval $[0,1]$.

sock: cyl

generates a Sock Diagram in three-dimensional cylindrical coordinates (default setting), p. 32. The frequency is the height axis, the phase is the azimuth angle, and the radial coordinate refers to the sock significance.

sock:fill <double>(0)

specifies a filling factor to compute extra frequencies if the sock significance difference between two adjacent frequencies is too high, p. 32.

parameter: number of additional frequencies per unit sig (difference between two adjacent frequencies)

sock:phases <int> ()

generates a Sock Diagram for the sampling of the given time series, p. 31. By default, no Sock Diagram is computed.

parameter: number of phase angles in the interval $[0, \pi[$, if the maximum sock significance is $\leq 1$. Between 1 and 2 , twice this number is used, and so on. This enhances the visibility of the Sock Diagram also in frequency and phase regions associated with a high sock significance. 
spectra <int> <int> ()

output files containing spectra (only s000000. dat for the spectrum of the initial time series and resspec.dat for the spectrum of the residuals after prewhitening all significant compontents by default). The parameters are

1. the maximum number of iterations (files s\#iteration\#.dat), and

2. the step width (number of iterations) for output, p. 26.

The file name may be assigned additional indices for Time-Resolved analysis (p. 42) and/or MultiFile mode (p. 56).

target <int> ()

specifies the file indicated by the parameter as target dataset, p. 59

timeres:range <double> ()

subset interval width [time units], p. 42

timeres:step <double> ()

step width between subset centres [time units], p. 42

timeres:w:cos <double><double> ()

cosine weights, p. 44

parameters:

- frequency [inverse time units]

- phase [rad]

timeres:w: cosp <double> <double> <double> ()

weights according to the power of a cosine, p. 44

parameters:

- frequency [inverse time units]

- phase [rad]

- exponent 
timeres:w:damp <double>()

exponential damping, p. 44

parameter: width [time units]

timeres:w:exp <double>()

exponential weights, p. 44

parameter: width [time units]

timeres:w: gauss <double>()

Gaussian weights, p. 44

parameter: standard deviation [time units]

timeres:w:ipow <double>()

inverse power weights, p. 44

parameter: exponent

timeres:w:none

unweighted moving averages, i. e. a rectangular filter, p. 44

ufreq <double> ()

upper frequency limit [inverse time units], p. 19

win

SigSPEC generates a file win. dat containing the spectral window for the given time series. By default, the file win.dat is not generated, p. 29.

\section{Online availability}

The ANSI-C code is available online at http://www. sigspec.org. For further information, please contact P. Reegen, peter.reegen@univie.ac.at.*

Acknowledgments. PR received financial support from the Fonds zur Förderung der wissenschaftlichen Forschung (FWF, projects P14546-PHY, P17580$\mathrm{N} 2$ ) and the BM:BWK (project COROT). Furthermore, it is a pleasure to thank T. Appourchaux (IAS, Orsay), A. Baglin (Obs. de Paris, Meudon),

\footnotetext{
*Please contact Michael Gruberbauer, mgruberbauer@ap.smu.ca.
} 
T. Boehm (Obs. M.-P., Toulouse), M. Breger, R. Dvorak, M. G. Firneis, D. Frast (Univ. of Vienna), R. Garrido (Inst. Astrof. Andalucia, Granada), M. Gruberbauer (Univ. of Vienna), D. B. Guenther (St. Mary's Univ., Halifax), M. Hareter, D. Huber, T. Kallinger (Univ. of Vienna), R. Kuschnig (UBC, Vancouver), S. Marchenko (Western Kentucky Univ., Bowling Green, KY), M. Masser (Univ. of Vienna), J.M. Matthews (UBC, Vancouver), E. Michel (Obs. de Paris, Meudon), A. F. J. Moffat (Univ. de Montreal), E. Paunzen, D. Punz (Univ. of Vienna), V. Ripepi (INAF, Naples), S. M. Rucinski (D. Dunlap Obs., Toronto), T. A. Ryabchikova (Inst. Astrpn. RAS, Moscow), D. Sasselov (Harvard-Smithsonian Center, Cambridge, MA), S. Schraml (Univ. of Technology, Vienna), G. A. Wade (Royal Military College, Kingston), G. A. H. Walker (UBC, Vancouver), W. W. Weiss, and K. Zwintz (Univ. of Vienna) for valuable discussion and support with extensive software tests. I acknowledge the anonymous referee for a detailed examination of both this publication and the corresponding software, as well as for the constructive feedback that helped to improve the overall quality a lot. Finally, I address my very special thanks to J. D. Scargle for his valuable support.

\section{References}

Breger, M., Stich, J., Garrido, R., et al. 1993, A\&A, 271, 482

Breger, M., Rucinski, S. M., Reegen, P. 2007, AJ, 134, 1994

Kallinger, T., Reegen, P., Weiss, W. W. 2008, A\&A, 481, 571

Kjeldsen, H., Bedding, T. R., Viskum, M., Frandsen, S. 1995, AJ, 109, 1313

Lomb, N. R. 1976, ApSS, 39, 447

Reegen, P. 2005, in The A-Star Puzzle, Proceedings of IAUS 224, eds. J. Zverko, J. Ziznovsky, S.J. Adelman, W.W. Weiss (Cambridge: Cambridge Univ. Press), p. 791 Reegen, P. 2007, A\&A, 467, 1353

Scargle, J. D. 1982, ApJ, 263, 835

Strassmeier, K. G., Boyd, L. J., Epand, D. H., Granzer, T. 1997, PASP, 109, 697

Zwintz, K., Marconi, M., Kallinger, T., Weiss, W. W. 2004, in The A-Star Puzzle, Proceedings of IAUS 224, eds. J. Zverko, J. Ziznovsky, S. J. Adelman, W. W. Weiss (Cambridge: Cambridge Univ. Press), p. 353

Zwintz, K., Weiss, W.W. 2006, A\&A, 457, 237 
